\title{
Predicting recurrent preeclampsia : empirical and methodological studies
}

Citation for published version (APA):

van Kuijk, S. M. J. (2013). Predicting recurrent preeclampsia : empirical and methodological studies. [Doctoral Thesis, Maastricht University]. Universitare Pers Maastricht. https://doi.org/10.26481/dis.20131217sk

Document status and date:

Published: 01/01/2013

DOI:

10.26481/dis.20131217sk

Document Version:

Publisher's PDF, also known as Version of record

\section{Please check the document version of this publication:}

- A submitted manuscript is the version of the article upon submission and before peer-review. There can be important differences between the submitted version and the official published version of record. People interested in the research are advised to contact the author for the final version of the publication, or visit the DOI to the publisher's website.

- The final author version and the galley proof are versions of the publication after peer review.

- The final published version features the final layout of the paper including the volume, issue and page numbers.

Link to publication

\footnotetext{
General rights rights.

- You may freely distribute the URL identifying the publication in the public portal. please follow below link for the End User Agreement:

www.umlib.nl/taverne-license

Take down policy

If you believe that this document breaches copyright please contact us at:

repository@maastrichtuniversity.nl

providing details and we will investigate your claim.
}

Copyright and moral rights for the publications made accessible in the public portal are retained by the authors and/or other copyright owners and it is a condition of accessing publications that users recognise and abide by the legal requirements associated with these

- Users may download and print one copy of any publication from the public portal for the purpose of private study or research.

- You may not further distribute the material or use it for any profit-making activity or commercial gain

If the publication is distributed under the terms of Article $25 \mathrm{fa}$ of the Dutch Copyright Act, indicated by the "Taverne" license above, 


\section{Predicting recurrent preeclampsia}

Empirical and methodological studies 
The research described in this thesis was funded by the Netherlands Organisation for Health Research and Development (ZonMw grant nr. 170882303).

The research presented in this thesis was conducted at the Department of Epidemiology, Maastricht University, and the Department of Obstetrics and Gynaecology, University Medical Centre Maastricht.

Financial support by the Stichting HELLP-syndroom for the publication of this thesis is gratefully acknowledged. Additional financial support was provided by BMA BV (Mosos).

ISBN 9789461592774

Cover design: Leandra Mackintosh, Mackintosh the Creative @mpany

Lay-out: Yvonne Leenders

Printed by: Datawyse

Copyright (C) 2013, S.M.J. van Kuijk, Heerlen 


\title{
Predicting recurrent preeclampsia Empirical and methodological studies
}

PROEFSCHRIFT

\author{
ter verkrijging van de graad van doctor aan de Universiteit Maastricht, \\ op gezag van de Rector Magnificus, Prof. dr. L.L.G. Soete \\ volgens het besluit van het College van Decanen, \\ in het openbaar te verdedigen \\ op dinsdag 17 december 2013 om 14:00 uur
}

door

Sander Martijn Job van Kuijk

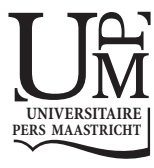




\section{Promotores}

Prof. dr. M.H. Prins

Prof. dr. M.E.A. Spaanderman

\section{Copromotores}

Dr. L.J.M. Smits

Dr. L.L.H. Peeters (UMC Utrecht)

\section{Beoordelingscommissie}

Prof. dr. H. ten Cate (voorzitter)

Prof. dr. P.C. Dagnelie

Dr. S. Koenen (UMC Utrecht)

Prof. dr. F.K. Lotgering (UMC St Radboud Nijmegen)

Prof. dr. E.W. Steyerberg (Erasmus MC Rotterdam) 


\section{Contents}

$\begin{array}{lll}\text { Chapter } 1 \text { General introduction } & 7\end{array}$

Part 1 Prediction of recurrent early-onset preeclampsia

Chapter 2 A model for preconceptional prediction of recurrent early-onset preeclampsia: derivation and internal validation

Chapter 3 Cost-effectiveness of recurrence risk guided care versus care as usual in women who suffered from early-onset preeclampsia in their previous pregnancy

Chapter 4 External validation of a model for preconceptional prediction of recurrent early-onset preeclampsia

Chapter 5 Multicentre impact analysis of a model for predicting recurrent early-onset preeclampsia

\section{Part 2: $\quad$ Methodology and statistics regarding prediction studies}

Chapter 6 How long do preconception risk prediction models hold?

Chapter 7 Index event bias - a numerical example

Chapter 8 Bias in regression coefficient estimates when assumptions for handling missing data are violated

Chapter 9 Summary and general discussion

Nederlandstalige samenvatting

Dankwoord

Curriculum vitae 



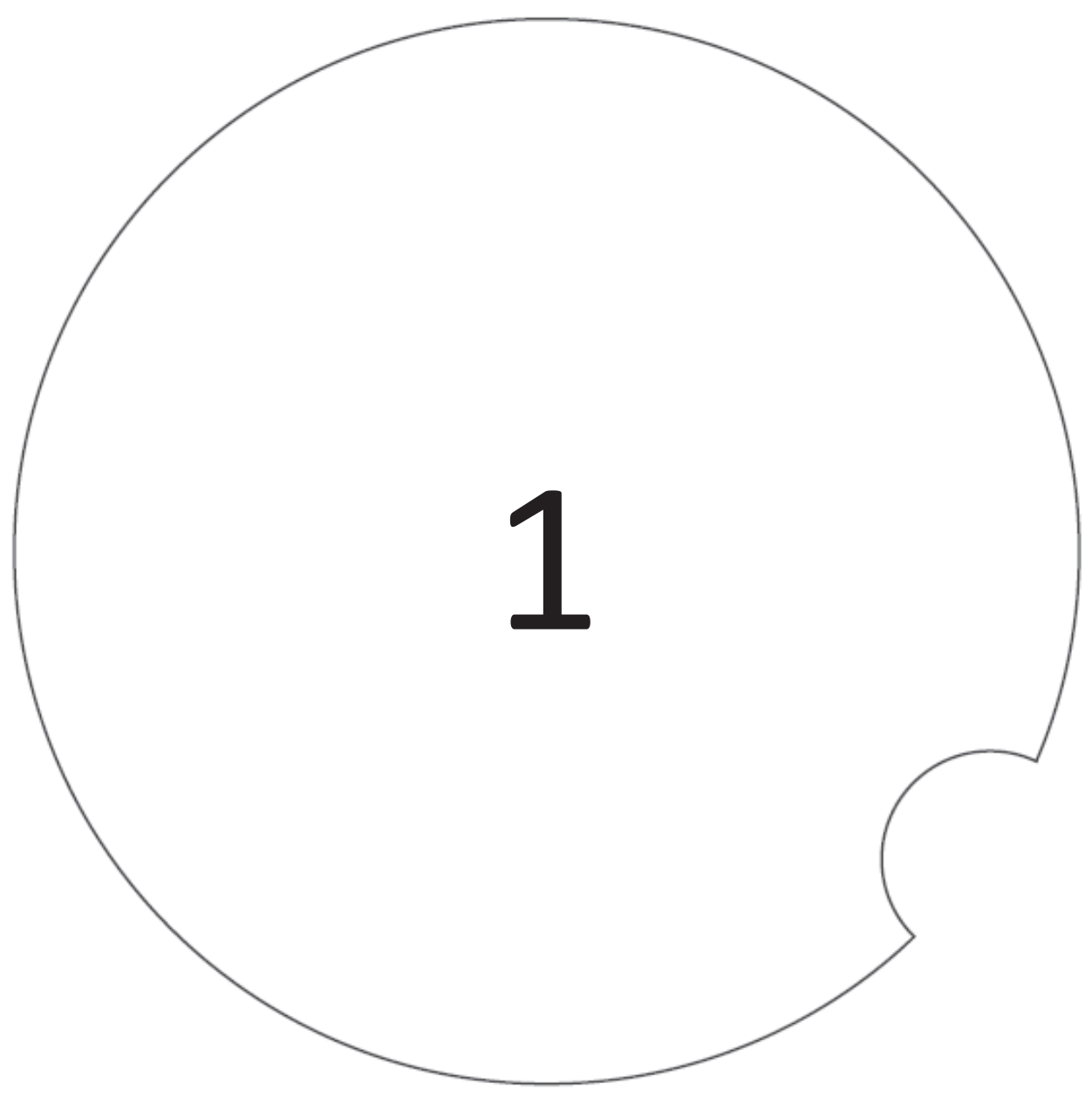

General introduction 


\section{Part 1: Prediction of recurrent early-onset preeclampsia}

\section{Preeclampsia and recurrence}

Preeclampsia is a hypertensive pregnancy disorder defined by either de novo hypertension (systolic blood pressure (BP) $\geq 140 \mathrm{mmHg}$ and/or diastolic BP $\geq 90 \mathrm{mmHg}$ ) or pre-existing chronic hypertension, accompanied by de novo proteinuria ( $\geq 300 \mathrm{mg} /$ day urinary protein excretion) developing after 20 weeks amenorrhea ${ }^{1,2}$. It may be paralleled by maternal symptoms such as headache, nausea, vomiting, visual disturbance, and epigastric pain ${ }^{2,3}$. Preeclampsia complicates approximately $2-8 \%$ of all first pregnancies ${ }^{4-7}$ and is a major cause of maternal and foetal morbidity and mortality, especially in case of early disease onset. Early-onset preeclampsia, defined as preeclampsia resulting in delivery before 34 weeks of gestational age, occurs in about $10 \%$ of all cases ${ }^{8}$.

Since the incidence of preeclampsia in parous women is much lower, it is often defined as a disease of first pregnancies. Nevertheless, women with a history of preeclampsia are at increased risk of preeclampsia in subsequent pregnancies. After a previous early-onset preeclampsia, the risk of developing recurrent early-onset disease in a next pregnancy amounts to about $7 \%{ }^{4}$, which is approximately 60 -fold as high as the risk in women who had a normotensive first pregnancy. In these women, preeclampsia irrespective of gestational age at onset recurs in $25-29 \%{ }^{4,9,10}$. If preeclampsia does recur, it is often more severe than preeclampsia in nulliparous women ${ }^{11}$.

\section{Management of subsequent pregnancy after preeclampsia}

In the Netherlands, no guidelines are available for the management of a next pregnancy in women with a history of early-onset preeclampsia. Recent studies suggest that the first step in patient management is the identification of risk factors for recurrent disease ${ }^{12,13}$. This can be either incorporated in a pre-pregnancy counselling visit or at booking for the next pregnancy. Being informed about a patients' individual recurrence risk may have consequences for clinical management. Women at low recurrence risk may be reassured and offered simpler and less frequent antenatal care to prevent superfluous medicalization. On the other hand, women at high recurrence risk may be offered more extensive and more frequent outpatient visits than their lowrisk counterparts. Tailored to the findings at each antenatal visit they may be subjected to additional testing, such as more frequent foetal ultrasound and Doppler measurements and the serial measurements of circulating levels of pIGF and/or Flt-1. This may enable the detection of recurrence already in the subclinical phase. If the proportion of women with minimal recurrence risk is large and reliably detectable, healthcare costs savings may be significant because of a lower number of outpatient visits for low-risk women, receiving less superfluous testing. In addition, it restricts the use of expensive targeted health care to those who are likely to benefit most.

Effects of recurrence-risk communication and risk-based antenatal care strategies on outcomes of subsequent pregnancies are still unknown. Women at low risk of recurrence who are reassured and offered routine antenatal care may be less anxious, less stressed, and may anticipate an uneventful next pregnancy compared to women 
who do not know their individual risk of recurrence. However, it may also induce these feelings of anxiety and stress, especially if the previous pregnancy is seen as a traumatic event. Meanwhile, women who have a high risk of recurrence of preeclampsia can be offered all necessary care and attention, despite the initial distress resulting from the consultation when they were informed about their elevated risk.

The possibilities to prevent recurrent preeclampsia are limited ${ }^{14}$, and risk-based tailored care is unlikely to have an influence on the rates of recurrence in low or highrisk groups. However, secondary prevention strategies may be beneficial for high-risk women, if disease onset can be identified at an earlier gestational age. This would not only allow the earliest possible start of treatment with e.g. antihypertensive drugs, potentially delaying disease progression. It would also identify the women who are likely to benefit from early intensified monitoring of their condition and that of their unborn child. To study the effects of recurrence-risk guided care on patient management and clinical outcomes, first a prediction model is needed to estimate the individual recurrence risk. The individual patient's risk estimate would enable the differentiation between women with a low risk and those with a high risk of recurrence.

\section{Risk factors for preeclampsia recurrence}

Many established risk factors for the occurrence of preeclampsia have been reported and include, among other factors, short period of exposure to the sperm of the father, extremes of maternal age, obesity and insulin resistance, and high blood pressure at booking or chronic hypertension ${ }^{15-17}$. Some studies have identified risk factors for recurrent preeclampsia in subsequent pregnancies ${ }^{4,18-22}$. Repeatedly reported candidate predictors are gestational age at delivery of the first complicated pregnancy, chronic hypertension, plasma volume, maternal age, ethnicity, and blood pressure at the start of the next pregnancy. However, no multivariable prediction models have been developed specifically to estimate the recurrence risk of early-onset preeclampsia. Such a model is necessary to combine the information from several observed risk factors into one risk estimate.

\section{Prediction models}

Prediction models are frequently developed and employed for clinical usage. They can be used for many tasks, including risk stratification, classification of disease status, or patient counselling. A desirable characteristic of prediction models is that such models provide an estimate of an individual's risk of the outcome in question, compared to only relative measures, such as odds ratios, hazard ratios, or risk ratios, for variables contained in the model. Prediction models can be used to estimate the risk on a scale from 0 to $100 \%$, but can easily be categorized or dichotomized for clinical decisionmaking. 


\section{Development of a prediction model}

The development of a risk prediction model consists of a number of phases, i.e. development, internal- and external validation and analysis of impact ${ }^{23-27}$. In the first phase regression coefficients of predictor variables can be estimated that are preferably selected a priori, or at least not selected based on p-values obtained in the same dataset because of the probability of overestimating coefficients ${ }^{24,28-32}$. In the second phase, after having estimated the model's coefficients, and in the absence of a suitable dataset for external validation, the model can be internally validated.

The internal validation phase serves to assess the extent of overfitting. This occurs when a prediction model yields good predictions on the data used to estimate the model, but its performance is reduced when applied to future patients. Some internal validation methods even provide shrinkage factors for penalizing regression coefficients. These factors range from 0 to 1 and are used to multiply the regression coefficients by. After penalizing the regression coefficients, the model makes less extreme predictions (i.e. closer to the mean risk) ${ }^{33-36}$. The bootstrap ${ }^{37}$ is a commonly used internal validation method for developing prediction models. To this end, a bootstrap sample is obtained by randomly drawing patients from a dataset with replacement, until the bootstrap sample has the same size of the original dataset. Because of sampling with replacement, some patients will not be selected for a given bootstrap sample, others will be selected one or more times. This is repeated $B$ times, which is often a few hundred or thousand times, and mimics the process of repeated sampling from the underlying population ${ }^{24}$. Results from the bootstrap samples are used to estimate future model performance and to determine the shrinkage factor.

Before widespread implementation of an internally validated model, its external validity and transportability to other populations has to be demonstrated. For this phase, the model should be applied to new, preferably prospectively-collected data. The model's performance in such a new cohort is likely to be comparable to that in future patients from comparable populations as the one used for the external validation.

A prediction model aimed at clinical decision-making requires completion of a fourth development phase. This phase consists of the evaluation of the impact on clinical outcome of implementing the prediction model in clinical practice ${ }^{38-41}$. If the individual risk estimate alters patient management, outcomes such as comorbidities, quality of life and psychological endpoints can be compared to a situation in which no risk prediction model is used. Results of such an impact analysis can help decide whether a model can be safely and effectively implemented in various other clinical settings, and result in better patient care. 


\section{Part 2: Methodology and statistics regarding prediction studies}

Several potential pitfalls can be identified in the course of developing and validating risk prediction models. Some of these pitfalls are unique to developing prediction models, others also apply to most other types of empirical research. The paragraphs below provide an elaboration on three examples.

\section{Validating prediction models for avoidable risks}

One characteristic of a particular pregnancy outcome is the possibility to "choose" for being at risk for that outcome. Modifying lifestyle or adopting a certain prophylactic compound may reduce the probability of developing pregnancy complications. An example for the latter is the use of folic acid supplementation in the first trimester of pregnancy to minimize the risk of having a baby with a neural tube defect. However, such a strategy does not abolish the chance of having that particular outcome, unless a woman decides not to conceive anymore. The latter applies to a woman who decides to refrain from future progeniture because of her increased risk for recurrent preeclampsia. This behaviour can be seen as a variant of selective fertility, defined as the tendency to adjust reproduction because of an adverse outcome of a previous pregnancy ${ }^{42}$. When such a choice is based on the result of a prediction model estimates, the use of such a model alters the potential validation population, and may distort the results of an external validation study using data from a prospective cohort of women who received pre-pregnancy counselling. The effect of this type of selection bias on the performance of a prediction model in such a sample is unknown.

\section{Introducing bias by selecting patients based on previous disease}

Pregnant women with a history of early-onset preeclampsia constitute a risk group for recurrence, and part of this thesis is dedicated to estimate associations between plausible predictors and recurrence, and combine these predictors in one multivariable prediction model. In studies of risk factors for recurrence, the study cohort is usually confined to women who experienced the target event in a preceding pregnancy and therefore are at risk for recurrence. Associations between risk factors and recurrence are often reported to be much closer to zero or even inversed when compared to studies of first disease occurrence. These sometimes paradoxical findings are inherent to the selection of patients, at forehand already known to have a higher prevalence of well-known risk factors. In this particular subpopulation, unknown risk factors contribute most to disease recurrence. Therefore, one should keep in mind that such a scenario may prevail in the exploration of recurrence risk of preeclampsia, and thus may introduce bias in the estimates of associations between the variables in the model and recurrence risk. The mechanism of this bias is known as Index event bias ${ }^{43,44}$, which is in its more general form known as Berkson's bias ${ }^{45}$ or collider (stratification) bias ${ }^{46}$. Although the theoretical principle of this type of bias in recurrence-risk research has been described ${ }^{43}$, its effects on the model coefficients and the risk of recurrence have not been quantified. 


\section{Imputing data when assumptions are unclear}

The third example of a potential pitfall that may have a sizeable impact on all empirical research is the problem of missing values. After years of gathering relevant patient information for a study, a researcher will almost inevitably conclude that some or many important variables are partly missing. Without specifying how to handle these missing values, most statistical packages will omit all subjects from the analysis with one or more values missing on variables that are used for the (multivariable) analysis. This may lead to an enormous loss of information and statistical power, but may also cause biased results if omitted patients are not a random selection of the total group $^{24,47-51}$. As a solution, some studies propose the use of multiple imputation techniques ${ }^{49,52,53}$. These usually regression or predictive mean matching based techniques provide data that can be analysed using standard regression techniques, which then give unbiased estimates of regression coefficients when certain assumptions are met, but the method is far from flawless ${ }^{54-56}$. Usually simulation studies adopt scenarios with these implicit assumptions being met, thus generating only positive results. This raises the question as to how multiple imputation performs when these assumptions are not met.

\section{Objectives}

The work in this thesis has three main objectives. The first objective was to develop and validate a prediction model that could be used for recurrence-risk stratification of pregnant women with a history of early-onset preeclampsia. The second objective was to assess the external validity of the model, and its impact when used it as a decision aid to stratify women into risk-based antenatal care strategies. The third objective was to study the implications of some of the main methodological and statistical issues that arise when performing prediction studies.

This thesis covers the development and impact of a prediction model. Studies of costs associated with risk-based antenatal care, cost-effectiveness of introducing this strategy in Dutch hospitals, and patient satisfaction, are not included in this thesis. These aspects of implementing a prediction model are the main objectives of the thesis by Denise Delahaije, researcher at the department of Clinical Epidemiology and Medical Technology Assessment, Maastricht University, Netherlands.

\section{Outline of this thesis}

\section{Part 1: Prediction of recurrent early-onset preeclampsia}

In Chapter 2, we present a model for the prediction of the individual risk of recurrent early-onset preeclampsia. This model is based on the medical and obstetrical history and is developed to be used during preconceptional counselling or at booking. Before widespread implementation, such a model should be externally validated and undergo an impact analysis. Chapter 3 describes the design of the so called PreCare study. In this study, both prospective and retrospective data were collected of pregnant women who had preeclampsia in their preceding pregnancy. In the first part of the study 
women received standard care according to the local guidelines. In the second part of the study the model was introduced and the participants received risk-based stratified antenatal care.

A subset of women of the data collected for the PreCare study was used for the external validation of the prediction model. They were recruited in a number of other hospitals besides the ones where the model had been developed. All validation efforts are described in Chapter 4 . Chapter 5 describes the impact of using the prediction model for risk-based stratification of antenatal care. This chapter describes the effect of implementing this model on patient care, including the impact on maternal and foetal outcomes.

\section{Part 2: Methodology and statistics regarding prediction studies}

In Chapter 6 we study the effect of this risk-based selection of women on performance of the prediction model in an external validation. We use a hypothetical example to demonstrate its effect on sensitivity and specificity for certain cut-of values of the predicted risk. Risk factors known to be important in the first occurrence of disease may not only lose strength in the risk assessment of recurrent disease, a positive association with recurrence risk may even have switched to an inverse relationship. If no plausible biological pathway exists, this may be related to index-event bias. Chapter 7 provides a numerical example of this type of bias and shows how it may develop and what its potential effect may be on estimates of risk factors.

Bias may also be a consequence of incomplete observation of data in the development of a prediction model. These missing data can be ignored, a scenario in which patients that have one or more values missing will be omitted from the analysis, or imputed, a scenario in which a missing value will be replaced with a plausible one. These methods have been shown to perform satisfactory when strict assumptions about the mechanism responsible for missing data are met. Chapter 8 shows results from a stochastic simulation study with the regression coefficients being estimated after omission or imputation of subjects from who part of important variables are missing, while also adherence to relevant assumptions was violated.

\section{Part 3: General discussion}

Chapter 9 summarizes the background, design and results of the studies contained in part I, discusses their implications and limitations, and gives some recommendations for further research. Furthermore, the findings and implications of the methodological studies of part II or discussed, and recommendations for future research are given. 


\section{References}

1. Brown MA, Lindheimer MD, de Swiet M, Van Assche A, Moutquin JM. The classification and diagnosis of the hypertensive disorders of pregnancy: statement from the International Society for the Study of Hypertension in Pregnancy (ISSHP). Hypertens Pregnancy. 2001;20(1):IX-XIV.

2. Sibai B, Dekker G, Kupferminc M. Pre-eclampsia. Lancet. Feb 26-Mar 4 2005;365(9461):785-799.

3. Thangaratinam S, Gallos ID, Meah N, Usman S, Ismail KM, Khan KS. How accurate are maternal symptoms in predicting impending complications in women with preeclampsia? A systematic review and meta-analysis. Acta Obstet Gynecol Scand. Jun;90(6):564-573.

4. Hernandez-Diaz S, Toh S, Cnattingius S. Risk of pre-eclampsia in first and subsequent pregnancies: prospective cohort study. Bmj. 2009;338:b2255.

5. Hauth JC, Ewell MG, Levine RJ, et al. Pregnancy outcomes in healthy nulliparas who developed hypertension. Calcium for Preeclampsia Prevention Study Group. Obstet Gynecol. Jan 2000;95(1):24-28.

6. Vatten LJ, Skjaerven R. Is pre-eclampsia more than one disease? Bjog. Apr 2004;111(4):298-302.

7. Witlin AG, Sibai BM. Magnesium sulfate therapy in preeclampsia and eclampsia. Obstet Gynecol. Nov 1998;92(5):883-889.

8. Lain $\mathrm{KY}$, Roberts JM. Contemporary concepts of the pathogenesis and management of preeclampsia. Jama. Jun 26 2002;287(24):3183-3186.

9. van Rijn BB, Hoeks LB, Bots ML, Franx A, Bruinse HW. Outcomes of subsequent pregnancy after first pregnancy with early-onset preeclampsia. Am J Obstet Gynecol. Sep 2006;195(3):723-728.

10. Bramham K, Briley AL, Seed P, Poston L, Shennan AH, Chappell LC. Adverse maternal and perinatal outcomes in women with previous preeclampsia: a prospective study. American Journal of Obstetrics and Gynecology. 6// 2011;204(6):512.e511-512.e519.

11. Hnat MD, Sibai BM, Caritis $S$, et al. Perinatal outcome in women with recurrent preeclampsia compared with women who develop preeclampsia as nulliparas. Am J Obstet Gynecol. Mar 2002;186(3):422-426.

12. Barton JR, Sibai BM. Prediction and prevention of recurrent preeclampsia. Obstet Gynecol. Aug 2008;112(2 Pt 1):359-372.

13. Dildy GA, 3rd, Belfort MA, Smulian JC. Preeclampsia recurrence and prevention. Semin Perinatol. Jun 2007;31(3):135-141.

14. Bezerra Maia EHMS, Marques Lopes L, Murthi P, da Silva Costa F. Prevention of preeclampsia. J Pregnancy. 2012;2012:435090.

15. Dekker GA. Risk factors for preeclampsia. Clin Obstet Gynecol. Sep 1999;42(3):422-435.

16. Duckitt K, Harrington D. Risk factors for pre-eclampsia at antenatal booking: systematic review of controlled studies. Bmj. Mar 12 2005;330(7491):565.

17. Knuist M, Bonsel GJ, Zondervan HA, Treffers PE. Risk factors for preeclampsia in nulliparous women in distinct ethnic groups: a prospective cohort study. Obstet Gynecol. Aug 1998;92(2):174-178.

18. Sep S, Smits L, Prins M, Peeters L. Prediction tests for recurrent hypertensive disease in pregnancy, a systematic review. Hypertens Pregnancy. Jan 2010;29(2):206-230.

19. Melamed N, Hadar E, Peled Y, Hod M, Wiznitzer A, Yogev Y. Risk for recurrence of preeclampsia and outcome of subsequent pregnancy in women with preeclampsia in their first pregnancy. 20121015 2012(1476-4954 (Electronic)).

20. McDonald SD, Best C, Lam K. The recurrence risk of severe de novo pre-eclampsia in singleton pregnancies: a population-based cohort. BJOG. Nov 2009;116(12):1578-1584. 
21. Bramham K, Briley AL, Seed P, Poston L, Shennan AH, Chappell LC. Adverse maternal and perinatal outcomes in women with previous preeclampsia: a prospective study. Am J Obstet Gynecol. Jun 2011;204(6):512 e511-519.

22. Sep SJ, Smits LJ, Prins MH, Spaanderman ME, Peeters LL. Simple prepregnant prediction rule for recurrent early-onset hypertensive disease in pregnancy. Reprod Sci. Jan 2009;16(1):80-87.

23. Moons KG, Royston $P$, Vergouwe $Y$, Grobbee DE, Altman DG. Prognosis and prognostic research: what, why, and how? Bmj. 2009;338:b375.

24. Steyerberg EW. Clinical Prediction Models. New York: Springer; 2009.

25. Jeremy CW, Douglas GA. Commentary: Prognostic models: clinically useful or quickly forgotten? BMJ. 1995;311.

26. Bouwmeester W, Zuithoff NP, Mallett S, et al. Reporting and methods in clinical prediction research: a systematic review. PLoS medicine. 2012;9(5):1-12.

27. Karel GMM, Patrick R, Yvonne V, Diederick EG, Douglas GA. Prognosis and prognostic research: what, why, and how? BMJ. 2009;338.

28. Harrell Jr FE. Regression Modeling Strategies. New York: Springer; 2001.

29. Wiegand RE. Performance of using multiple stepwise algorithms for variable selection. Stat Med. Jul 10 2010;29(15):1647-1659.

30. Steyerberg EW, Eijkemans MJ, Habbema JD. Stepwise selection in small data sets: a simulation study of bias in logistic regression analysis. $J$ Clin Epidemiol. Oct 1999;52(10):935-942.

31. Chatfield C. Model Uncertainty, Data Mining and Statistical Inference. Journal of the Royal Statistical Society. Series A (Statistics in Society). // 1995;158(3):419-466.

32. Vach K, Sauerbrei W, Schumacher M. Variable Selection and Shrinkage: Comparison of Some Approaches. Statistica Neerlandica. 2001;55(1):53-75.

33. Steyerberg EW, Eijkemans MJ, Harrell FE, Jr., Habbema JD. Prognostic modelling with logistic regression analysis: a comparison of selection and estimation methods in small data sets. Stat Med. Apr 30 2000;19(8):1059-1079.

34. Moons KG, Kengne AP, Woodward M, et al. Risk prediction models: I. Development, internal validation, and assessing the incremental value of a new (bio)marker. Heart (British Cardiac Society). May 2012;98(9):683-690.

35. Steyerberg EW, Bleeker SE, Moll HA, Grobbee DE, Moons KG. Internal and external validation of predictive models: a simulation study of bias and precision in small samples. $J$ Clin Epidemiol. May 2003;56(5):441-447.

36. Steyerberg EW, Harrell FE, Jr., Borsboom GJ, Eijkemans MJ, Vergouwe Y, Habbema JD. Internal validation of predictive models: efficiency of some procedures for logistic regression analysis. J Clin Epidemiol. Aug 2001;54(8):774-781.

37. Efron B. Bootstrap Methods: Another Look at the Jackknife.

38. McGinn TG, Guyatt GH, Wyer PC, Naylor CD, Stiell IG, Richardson WS. Users' guides to the medical literature: XXII: how to use articles about clinical decision rules. Evidence-Based Medicine Working Group. JAMA. Jul 5 2000;284(1):79-84.

39. Reilly BM, Evans AT. Translating clinical research into clinical practice: impact of using prediction rules to make decisions. Annals of internal medicine. Feb 7 2006;144(3):201209.

40. Toll DB, Janssen KJ, Vergouwe Y, Moons KG. Validation, updating and impact of clinical prediction rules: a review. J Clin Epidemiol. Nov 2008;61(11):1085-1094.

41. Moons KG, Altman DG, Vergouwe $Y$, Royston P. Prognosis and prognostic research: application and impact of prognostic models in clinical practice. BMJ. 2009;338:b606.

42. Skjaerven R, Wilcox AJ, Lie RT, Irgens LM. Selective fertility and the distortion of perinatal mortality. American journal of epidemiology. Dec 1988;128(6):1352-1363. 
43. Dahabreh IJ, Kent DM. Index event bias as an explanation for the paradoxes of recurrence risk research. JAMA. Feb 23 2011;305(8):822-823.

44. Smulders YM. [Index event bias: why causal factors appear not to apply to disease recurrence]. Nederlands tijdschrift voor geneeskunde. 2011;155:A3458.

45. Berkson J. Limitations of the application of fourfold table analysis to hospital data. Biometrics. Jun 1946;2(3):47-53.

46. Greenland S. Quantifying biases in causal models: classical confounding vs colliderstratification bias. Epidemiology. May 2003;14(3):300-306.

47. Rubin DB. Inference and missing data. Biometrika. December 1, 1976 1976;63(3):581-592.

48. Schafer JL. Analysis of incomplete multivatiate data. London: Chapman \&Hall/CRC Press; 1997.

49. van der Heijden GJ, Donders AR, Stijnen T, Moons KG. Imputation of missing values is superior to complete case analysis and the missing-indicator method in multivariable diagnostic research: a clinical example. J Clin Epidemiol. Oct 2006;59(10):1102-1109.

50. White IR, Carlin JB. Bias and efficiency of multiple imputation compared with completecase analysis for missing covariate values. Stat Med. Dec 10;29(28):2920-2931.

51. Knol MJ, Janssen KJ, Donders AR, et al. Unpredictable bias when using the missing indicator method or complete case analysis for missing confounder values: an empirical example. J Clin Epidemiol. Jul 2010;63(7):728-736.

52. Janssen KJ, Donders AR, Harrell FE, Jr., et al. Missing covariate data in medical research: to impute is better than to ignore. J Clin Epidemiol. Jul 2010;63(7):721-727.

53. Donders AR, van der Heijden GJ, Stijnen T, Moons KG. Review: a gentle introduction to imputation of missing values. J Clin Epidemiol. Oct 2006;59(10):1087-1091.

54. Allison PD. Missing Data. lowa: Sage; 2001.

55. Sterne JA, White IR, Carlin JB, et al. Multiple imputation for missing data in epidemiological and clinical research: potential and pitfalls. BMJ. 2009;338:b2393.

56. White IR, Carlin JB. Bias and efficiency of multiple imputation compared with completecase analysis for missing covariate values. Stat Med. Dec 10 2010;29(28):2920-2931. 


\section{Part 1: Development of a prediction model for the recurrence of early-onset preeclampsia}

"An unsophisticated forecaster uses statistics as a drunken man uses lamp-posts - for support rather than for illumination."

Andrew Lang

"It is said that the present is pregnant with the future"

Voltaire 


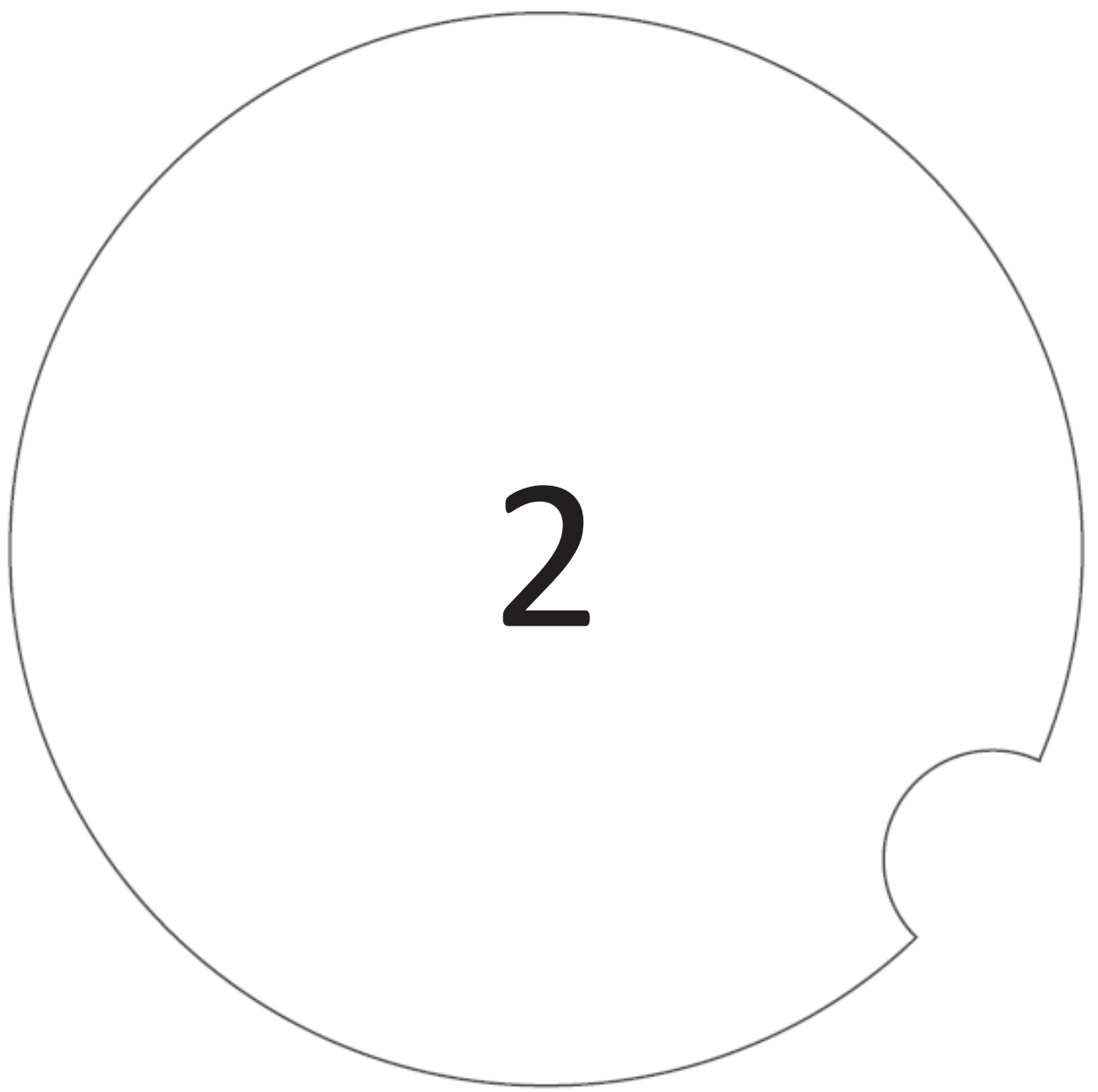

\section{A model for preconceptional prediction of recurrent early-onset preeclampsia: derivation and internal validation}

Van Kuijk SM, Nijdam ME, Janssen KJ, Sep SJ, Peeters LL, Delahaije DH, Spaanderman ME, Bruinse HW, Franx A, Bots ML, Langenveld J, van der Post J, van Rijn BB, Smits LJ Reproductive Sciences 2011 18: 1154 


\section{Abstract \\ Objective}

To develop a model to identify women at very low risk for a recurrence of early-onset preeclampsia.

\section{Methods}

We enrolled 407 women who had experienced early-onset preeclampsia in their first pregnancy, resulting in a delivery before 34 weeks gestation. Preeclampsia was defined as hypertension (systolic blood pressure $\geq 140 \mathrm{mmHg}$ and/ or diastolic blood pressure $\geq 90 \mathrm{mmHg}$ ) after 20 weeks' gestation with de novo proteinuria ( $\geq 300 \mathrm{mg}$ urinary protein excretion/day). Based on previous published evidence and expert opinion, five predictors (gestational age at previous birth, prior small-for-gestational-age newborn, fasting blood glucose, body mass index and hypertension) were entered in a logistic regression model. Discrimination and calibration were evaluated after adjusting for overfitting by bootstrapping techniques.

\section{Results}

Early-onset disease recurred in 28 (6.9\%) of 407 women. The area under the receiver operating characteristic (ROC) curve of the model was $0.65(95 \% \mathrm{Cl}: 0.56-0.74)$. Calibration was good, indicated by a non-significant Hosmer-Lemeshow test $(P=0.11)$. Using a predicted absolute risk threshold of, for example, $4.6 \%$ (i.e., women identified with an estimated risk either above or below $4.6 \%$ ), the sensitivity was $100 \%$, with a specificity of $26 \%$. In such a strategy, no women who developed preeclampsia were missed, while 98 of the 407 women would be regarded as low risk of recurrent earlyonset preeclampsia, not necessarily requiring intensified antenatal care.

\section{Conclusion}

Our model may be helpful in the identification of women at very low risk of recurrent early-onset preeclampsia. Before widespread application, our model should be validated in other populations. 


\section{Introduction}

Preeclampsia, including the syndrome of haemolysis, elevated liver enzymes and low platelets (HELLP), is a pregnancy-related hypertensive disorder which complicates $2-8 \%$ of all pregnancies in nulliparous women ${ }^{1-4}$, and accounts for approximately $10 \%$ of all pregnancy-related maternal mortality in developed countries ${ }^{5}$. Early-onset disease, occurring in about $10 \%$ of all cases ${ }^{6}$, is defined as preeclampsia resulting in delivery before 34 weeks' gestation. It can lead to serious maternal and perinatal complications ${ }^{7}$. The precise cause of preeclampsia is still unsettled, but the current idea favours a two-stage model of placental disease that initiates a maternal inflammatory syndrome causing generalized endothelial dysfunction ${ }^{8}$.

Although preeclampsia is mostly considered a disease of the first pregnancy, pregnant women with a history of preeclampsia are at increased risk ${ }^{9-12}$. Estimates of the rate of early-onset recurrence in women with previous preeclampsia are approximately 4$7 \%{ }^{1,7}$. Furthermore, the course of the disease is reported to be more severe among women with recurrent preeclampsia than among preeclamptic nulliparas ${ }^{13}$.

At present, there are no guidelines for the management of women with a history of early-onset preeclampsia. In clinical practice these women usually receive intensified surveillance, with far more than the routine antenatal visits, and additional testing like uterine artery Doppler studies and serial foetal ultrasound biometry and Doppler studies, and repeated biochemical testing. Yet, we have previously shown that only $25 \%$ of these women develop recurrent preeclampsia and only $5 \%$ develop recurrent early-onset preeclampsia ${ }^{7}$. Therefore, most women with a history of early-onset preeclampsia may be monitored safely by routine antenatal care, rather than intensified surveillance. Identification of women with a very low recurrence risk, even prior to the subsequent pregnancy, would decrease the burden of intensified surveillance for both the women and the antenatal care facilities and resources.

Recently, we published a prediction model to estimate the recurrence risk in women with preeclampsia, including HELLP, diagnosed before or at 34 weeks, resulting in delivery before 37 weeks' gestation ${ }^{14}$, thus including also the later and milder cases. The number of cases $(N=16)$ in this single-centre study was rather small, and thus precision and the general applicability of the model seems limited. In the present study we aimed to develop a preconceptional model for the prediction of recurrence of early-onset preeclampsia, including the HELLP-syndrome, resulting in delivery before 34 weeks' gestation. We used data of 407 women with a previous early-onset preeclampsia who delivered in any of five Dutch perinatal referral centres. 


\section{Patients and methods}

\section{Patients}

We combined five cohorts comprising women with early-onset preeclampsia, including the HELLP syndrome, in their first pregnancy, resulting in delivery before 34 weeks of gestation. These women had undergone subsequent postpartum screening and had a recorded subsequent ongoing pregnancy, following the index pregnancy that was complicated by early-onset preeclampsia. The maternal and neonatal outcomes of that second pregnancy were available. Only women with first and subsequent deliveries of singletons were included. Data were collected from five perinatal tertiary referral centres in the Netherlands (Maastricht University Medical Centre, University Medical Centre Utrecht, Academic Medical Centre Amsterdam, University Medical Centre St. Radboud Nijmegen and Máxima Medical Centre Veldhoven) between 1993 and 2008. The date of delivery of the subsequent pregnancy was used as the inclusion date.

In all five hospitals, preeclampsia was defined as de novo hypertension (systolic blood pressure (BP) $\geq 140 \mathrm{mmHg}$ and/ or diastolic $\mathrm{BP} \geq 90 \mathrm{mmHg}$ ) after 20 weeks' gestation along with de novo proteinuria ( $\geq 300 \mathrm{mg}$ urinary protein excretion/day), in accordance with the criteria of the International Society for the Study of Hypertension in Pregnancy (ISSHP) ${ }^{15}$. Preeclampsia superimposed on chronic hypertension was defined as de novo proteinuria complicating pre-existent hypertension after 20 weeks' gestation or a sudden increase in proteinuria or blood pressure in women whose hypertension had previously been well controlled ${ }^{15}$. The HELLP syndrome was defined as thrombocytopenia, haemolysis, and abnormal liver enzymes ${ }^{16}$. Cases were defined as women who had recurrent disease requiring delivery before 34 weeks' gestation.

\section{Preconceptional predictors}

Selected predictors of the recurrence risk of early-onset preeclampsia were chosen on the basis of previous studies ${ }^{17-19}$, and the availability of the predictors in all five cohorts. One gynaecologist was involved in the preselection of variables (LP), after which consensus was reached about the selection of variables among all expertgynaecologists within the author team. The list of selected predictors included items chosen from laboratory data obtained during postpartum screening, obstetrical history and medical history. Since the absolute number of women with a recurrent preeclampsia was small $(n=28)$, we allowed only 5 predictors to be included in the model in order to prevent severe overfitting with subsequently low generalizability ${ }^{20-}$ ${ }^{22}$. All predictors were measured when women were between their first index preterm pregnancy and their consecutive ongoing pregnancy.

One predictor was selected from laboratory tests, i.e. fasting blood glucose; predictors from obstetrical history and medical history were prior small-for-gestational-age (SGA) newborn, pregnancy duration of the first (index) pregnancy, body mass index (BMI) obtained during postpartum screening, and the presence or absence of hypertension. Maternal BMI and hypertension are widely accepted as predictors of preeclampsia ${ }^{10,23}$. Glucose can be seen as a proxy for insulin resistance, also a well-known predictor of preeclampsia ${ }^{9,24,25}$. Prior SGA newborn and a lower gestational age at the index 
pregnancy are known to be associated with a higher risk of recurrent preeclampsia ${ }^{17,19}$. Fasting blood glucose levels were measured after a 12-hour fasting period. Prior SGA newborn was defined as birth weight below the $10^{\text {th }}$ percentile for gestational age at delivery, based on the most recent Dutch population charts ${ }^{26}$. We defined hypertension as raised blood pressure (systolic blood pressure (BP) $\geq 140 \mathrm{mmHg}$ and/ or diastolic $\mathrm{BP} \geq 90 \mathrm{mmHg}$ ) or the use of antihypertensive medication before the second pregnancy.

\section{Data analysis}

Data were incomplete for some predictor variables. Glucose level was missing in $19.7 \%$ of the cases, maternal BMI in $10.1 \%$ of the cases, and chronic hypertension in $3.9 \%$ of the cases. Pregnancy outcomes were available for all subjects. We made the assumption that these data were missing at random (MAR), meaning that the probability of values being missing is deducible from values of other variables in the model, including the outcome variable ${ }^{27}$. We imputed these values by means of single regression imputation, since non-imputed complete case analyses often yield biased results ${ }^{28}$.

After imputation, the five selected predictors were entered in a multivariate logistic regression model. According to methodological recommendations ${ }^{29,30}$, the predictors remained in the prediction model irrespective of their statistical significance since the primary goal is the estimation of the individual risk of recurrence, not the estimation of regression coefficients or odds ratios.

Next, we assessed the performance of the prediction model by quantifying discrimination and calibration. Discrimination is the ability of the model to distinguish between women that develop early-onset preeclampsia and those who do not, quantified by the area under the receiver operating characteristic (ROC) curve ${ }^{31}$. The area under the ROC curve can range from 0.5 (no discrimination; in effect a flip of a coin) to 1.0 (perfect discrimination). Calibration refers to the agreement between the predicted probabilities and observed frequencies of recurrent preeclampsia. We evaluated calibration of the model by using the Hosmer-Lemeshow $(\mathrm{H}-\mathrm{L})$ goodness-offit test ${ }^{32}$. A high $\mathrm{H}-\mathrm{L}$ statistic is related to a low $\mathrm{p}$-value and indicates lack of fit. To reduce the risk of overfitting of the regression coefficients, a well-known phenomenon in predictive models ${ }^{22}$, we used bootstrapping techniques (i.e. repeating the entire modelling process) to internally validate the prediction model and adjust the regression coefficients by the resulting shrinkage factor ${ }^{33}$. The number of bootstrap samples we used was 200 . This internal validation step is undertaken to estimate the likely performance of the final model in new subjects, since external validation by splitsample would greatly reduce the sample size for the derivation of our model ${ }^{34}$. This does not make an external validation step obsolete, but corrects for a model's overfitting by making future predictions slightly less extreme. In addition, the discrimination and calibration of the adjusted prediction model were estimated. Finally, we constructed a table comparing sensitivity, specificity and positive and negative predictive values for different risk thresholds. 


\section{Results}

\section{Patient population}

The final study population consisted of 407 women with a history of early-onset preeclampsia and/or HELLP in their first pregnancy. Characteristics are shown in table 1. The overall recurrence of early-onset preeclampsia, including the HELLP-syndrome, was $6.9 \%$ (95\% confidence interval: $4.4-9.3$, in total 28 recurrences).

\section{Model development and performance}

Table 2 shows the parameters of the model. The initial regression coefficients were adjusted for overfitting using a shrinkage factor of 0.74 . The outcome of the model was the individual recurrence risk estimate, which ranged from 0 to $100 \%$. Discrimination of the model was $0.65(95 \% \mathrm{Cl}$ : $0.56-0.74)$, after correction for optimism by bootstrapping. The calibration of the model was good, confirmed by a non-significant Hosmer-Lemeshow test $(P=0.11)$.

Table 1 Characteristics of overall study population

\begin{tabular}{ll}
\hline Characteristic & Total group $\mathbf{( n = 4 0 7 )}$ \\
Recurrence early-onset preeclampsia and/or HELLP & $28(6.9)$ \\
Body mass index $\left(\mathrm{kg} / \mathrm{m}^{2}\right)$ & $25.7(4.7)$ \\
Hypertension, $n(\%)$ & $159(39.1)$ \\
Circulating level of glucose $(\mathrm{mmol} / \mathrm{L})$ & $5.0(0.8)$ \\
Characteristics of first pregnancy & \\
Gestational age at delivery (days) & $208.6(17.3)$ \\
Infant's birth weight $(\mathrm{g})$ & $1078.6(447.3)$ \\
SGA newborn $\left(<10^{\text {th }}\right.$ centile), $n(\%)$ & $146(35.9)$ \\
Age at first delivery $(\mathrm{yr})$ & $28.8(3.9)$ \\
\hline
\end{tabular}

Data are given as mean \pm SD or as percentages.

Table 2 Prediction model for the recurrence of early-onset preeclampsia ${ }^{a}$

\begin{tabular}{|c|c|c|c|}
\hline Variable & $\begin{array}{l}\text { Regression } \\
\text { Coefficient }^{b}\end{array}$ & Odds ratio & $95 \% \mathrm{Cl}$ \\
\hline Intercept & 0.29 & - & - \\
\hline Circulating level of glucose (mmol/L) & -0.42 & 0.66 & $(0.41-1.04)$ \\
\hline Hypertension (yes) & 0.59 & 1.80 & $(0.81-4.02)$ \\
\hline Gestational age at delivery (index pregnancy, days) & -0.01 & 0.99 & $(0.97-1.02)$ \\
\hline Prior small-for-gestational-age newborn (yes) & -0.41 & 0.66 & $(0.27-1.65)$ \\
\hline Maternal BMI $\left(\mathrm{kg} / \mathrm{m}^{2}\right)$ & 0.01 & 1.01 & $(0.93-1.10)$ \\
\hline
\end{tabular}

${ }^{a}$ To calculate the absolute risk of recurrence of early-onset preeclampsia:

(1 / (1+exp)-(0.29-0.42* circulating level of glucose $+0.59 *$ hypertension $-0.01 *$ gestational age at delivery $-0.41 *$ SGA $+0.01 *$ BMI) )) )*100\%

${ }^{\mathrm{b}}$ After adjustment for overfitting by shrinkage (shrinkage factor $=0.74$ )

Table 3 shows the clinical impact of using the model, with different thresholds for the predicted risk. Using a predicted risk threshold of, for instance, $4.6 \%$, sensitivity was 
highest (100\%), with a specificity of $26 \%$. From table 3 it can be seen that, when using a $4.6 \%$ risk threshold, no cases (i.e. women who develop preeclampsia before 34 weeks of gestation) would have been missed, while 98 of the 407 women would have been regarded as very low risk and might be referred back to usual antenatal care. When a risk threshold of $5.2 \%$ would be used, 144 of the 407 women would have been regarded as low risk, in which 3 cases of recurrent preeclampsia would have occurred. As exemplified in table 3, choosing a higher threshold leads to a higher specificity (less false positives), but at the cost of missing more women who will develop preeclampsia.

Table 3 Different thresholds of the predicted risk and the corresponding sensitivities and specificities and predictive values

\begin{tabular}{lcccc}
\hline Risk threshold $^{\mathrm{a}}$ & Sensitivity $(\%)^{\mathrm{b}}$ & ${\text { Specificity }(\%)^{\mathrm{b}}}$ & PPV $(\%)^{\mathrm{b}}$ & ${\mathrm{NPV}(\%)^{\mathrm{b}}}^{\mathrm{b}}$ \\
\hline 4.6 & 100 & 26 & 9 & 100 \\
& $(28 / 28)$ & $(98 / 379)$ & $(28 / 309)$ & $(98 / 98)$ \\
5.2 & 89 & 37 & 10 & 98 \\
& $(25 / 28)$ & $(141 / 379)$ & $(25 / 263)$ & $(141 / 144)$ \\
5.4 & 82 & 41 & 9 & 97 \\
& $(23 / 28)$ & $(157 / 379)$ & $(23 / 245)$ & $(157 / 162)$ \\
6.2 & 75 & 54 & 11 & 97 \\
& $(21 / 28)$ & $(206 / 379)$ & $(21 / 194)$ & $(206 / 213)$ \\
\hline
\end{tabular}

${ }^{a}$ Women were considered to have screened positive if the predicted risk (expressed as percentage) was at or above this level.

${ }^{b}$ Data in parentheses represent proportions.

Since both the positive and negative predictive values are dependent on the incidence in the population the prediction model is applied to, we performed a sensitivity analysis to assess the change in predictive values using the lower and the upper bound of the $95 \%$ confidence interval of the incidence of overall recurrence. Table 4 shows that, for a risk cut-off value of $5.2 \%$ and an incidence of recurrent preeclampsia of $9.3 \%$, the negative predictive value still remains very high $(97.0 \%)$, indicating that of the 100 patients with a negative test result, on average only 3 patients will show recurrence.

Table 4 The effect of preeclampsia recurrence on the estimates of predictive values, for a risk threshold of $5.2 \%$

\begin{tabular}{lcccc}
\hline Incidence & Sensitivity (\%) & Specificity (\%) & PPV (\%) & NPV (\%) \\
\hline Sample (6.9\%) & 82 & 41 & 10 & 98 \\
LB $^{* * *}(4.4 \%)$ & 82 & 41 & 6.1 & 98.7 \\
UB $^{* * 1}(9.3 \%)$ & 82 & 41 & 12.7 & 97.0 \\
\hline
\end{tabular}

"Women were considered to have screened positive if the predicted risk (expressed as percentage) was at or above this level.

${ }^{* *}$ LB and UB denote the lower bound and upper bound of the $95 \%$ confidence interval of the estimate of overall recurrence, respectively. 


\section{Discussion}

Although the risk of recurrence is generally low, women with a history of early-onset preeclampsia often undergo intensified surveillance in a next pregnancy, including far more than the usual antenatal visits and additional testing, like uterine artery Doppler studies and serial foetal ultrasound biometry and Doppler studies, and repeated biochemical testing. The prediction model presented in this report enables the identification of women who are at such a low risk of recurrent preeclampsia, that they do not need such intensified surveillance and can be reassured and safely offered routine antenatal care. This would not only decrease the burden of intensified surveillance for the women, but also for the antenatal care facilities and resources.

Our prediction model included predictors based on the literature as known factors related to increased risk and that can be obtained routinely during postpartum counselling. However, in our data we found inverse associations of fasting blood glucose level and prior SGA newborn with risk of recurrent disease. Also, the association between maternal $\mathrm{BMI}$ and the risk of recurrent preeclampsia was much weaker than reported for first occurrences of preeclampsia. These unexpected associations might be the effect of our study's confinement to women with previous preeclampsia, resulting in a phenomenon described earlier as collider bias ${ }^{35,36}$.

We developed the prediction model according to the latest methodological guidelines, which do not prescribe that predictors included in the model should show a statistical significant relation with the outcome $\mathrm{s}^{34,37}$. Actually, when only significant parameters are used, models become overfitted and usually perform poorly in other cohorts ${ }^{37}$.

Some drawbacks of this study also merit discussion. First, despite the combination of datasets, the study population and, more in particular, the number of early-onset preeclamptic events in the subsequent pregnancy were limited, thereby restricting the number of predictors that could be validly included in the model. Second, a potential drawback of combining datasets is that we could only include predictors that were measured in all five hospitals. Some known risk factors for the recurrence of preeclampsia which may have added predictive value could not be evaluated because they were not measured. They might have increased the discriminative ability of the model. Examples of these not universally measured but potential risk factors are maternal thrombophilia ${ }^{38}, \mathrm{HDL}$ cholesterol $^{14}$, plasma volume $^{39}$, and homeostasis model assessment of insulin resistance (HOMA-IR). However, the individual contribution of these factors in risk prediction needs to be evaluated further.

The model was validated internally. Yet, we highly encourage colleague researchers to validate the model and its thresholds for discriminating between very low- and highrisk women using their own data (external validation). Indeed, before widespread application, our model should be validated in other populations. We have recently started a validation study among patients of six university hospitals and seven large non-university tertiary referral hospitals in the Netherlands ${ }^{40}$. A next step would be to 
evaluate the effects of applying the prediction model on maternal and neonatal outcome, patient satisfaction, and health care expenses.

In summary, we developed and internally validated a prediction model for prepregnancy identification of women who are at very low risk for a recurrence of early onset preeclampsia in a subsequent pregnancy. 


\section{References}

1. Hernandez-Diaz S, Toh S, Cnattingius S. Risk of pre-eclampsia in first and subsequent pregnancies: prospective cohort study. Bmj. 2009;338:b2255.

2. Hauth JC, Ewell MG, Levine RJ, et al. Pregnancy outcomes in healthy nulliparas who developed hypertension. Calcium for Preeclampsia Prevention Study Group. Obstet Gynecol. Jan 2000;95(1):24-28.

3. Vatten LJ, Skjaerven R. Is pre-eclampsia more than one disease? Bjog. Apr 2004;111(4):298-302.

4. Witlin AG, Sibai BM. Magnesium sulfate therapy in preeclampsia and eclampsia. Obstet Gynecol. Nov 1998;92(5):883-889.

5. Khan KS, Wojdyla D, Say L, Gulmezoglu AM, Van Look PF. WHO analysis of causes of maternal death: a systematic review. Lancet. Apr 1 2006;367(9516):1066-1074.

6. Lain KY, Roberts JM. Contemporary concepts of the pathogenesis and management of preeclampsia. Jama. Jun 26 2002;287(24):3183-3186.

7. van Rijn BB, Hoeks LB, Bots ML, Franx A, Bruinse HW. Outcomes of subsequent pregnancy after first pregnancy with early-onset preeclampsia. Am J Obstet Gynecol. Sep 2006;195(3):723-728.

8. Redman CW, Sargent IL. Latest advances in understanding preeclampsia. Science. Jun 10 2005;308(5728):1592-1594.

9. Dekker GA. Risk factors for preeclampsia. Clin Obstet Gynecol. Sep 1999;42(3):422-435.

10. Sibai B, Dekker G, Kupferminc M. Pre-eclampsia. Lancet. Feb 26-Mar 4 2005;365(9461):785-799.

11. Duckitt K, Harrington D. Risk factors for pre-eclampsia at antenatal booking: systematic review of controlled studies. Bmj. Mar 12 2005;330(7491):565.

12. Wagner LK. Diagnosis and management of preeclampsia. Am Fam Physician. Dec 15 2004;70(12):2317-2324.

13. Hnat MD, Sibai BM, Caritis $S$, et al. Perinatal outcome in women with recurrent preeclampsia compared with women who develop preeclampsia as nulliparas. Am J Obstet Gynecol. Mar 2002;186(3):422-426.

14. Sep SJ, Smits LJ, Prins MH, Spaanderman ME, Peeters LL. Simple prepregnant prediction rule for recurrent early-onset hypertensive disease in pregnancy. Reprod Sci. Jan 2009;16(1):80-87.

15. Brown MA, Lindheimer MD, de Swiet M, Van Assche A, Moutquin JM. The classification and diagnosis of the hypertensive disorders of pregnancy: statement from the International Society for the Study of Hypertension in Pregnancy (ISSHP). Hypertens Pregnancy. 2001;20(1):IX-XIV.

16. Sibai BM. Diagnosis, controversies, and management of the syndrome of hemolysis, elevated liver enzymes, and low platelet count. Obstet Gynecol. May 2004;103(5 Pt 1):981-991.

17. Barton JR, Sibai BM. Prediction and prevention of recurrent preeclampsia. Obstet Gynecol. Aug 2008;112(2 Pt 1):359-372.

18. Dildy GA, 3rd, Belfort MA, Smulian JC. Preeclampsia recurrence and prevention. Semin Perinatol. Jun 2007;31(3):135-141.

19. Rasmussen S, Irgens LM, Albrechtsen S, Dalaker K. Predicting preeclampsia in the second pregnancy from low birth weight in the first pregnancy. Obstet Gynecol. Nov 2000;96(5 Pt 1):696-700.

20. Peduzzi P, Concato J, Kemper E, Holford TR, Feinstein AR. A simulation study of the number of events per variable in logistic regression analysis. J Clin Epidemiol. Dec 1996;49(12):1373-1379. 
21. Moons KG, Royston P, Vergouwe $\mathrm{Y}$, Grobbee DE, Altman DG. Prognosis and prognostic research: what, why, and how? Bmj. 2009;338:b375.

22. Royston $P$, Moons KG, Altman DG, Vergouwe $Y$. Prognosis and prognostic research: Developing a prognostic model. Bmj. 2009;338:b604.

23. O'Brien TE, Ray JG, Chan WS. Maternal body mass index and the risk of preeclampsia: a systematic overview. Epidemiology. May 2003;14(3):368-374.

24. Montoro MN, Kjos SL, Chandler M, Peters RK, Xiang AH, Buchanan TA. Insulin resistance and preeclampsia in gestational diabetes mellitus. Diabetes Care. Aug 2005;28(8):19952000.

25. Scioscia M, Gumaa K, Rademacher TW. The link between insulin resistance and preeclampsia: new perspectives. J Reprod Immunol. Jul 212009.

26. The Netherlands Perinatal Registery [homepage on the internet]. Bilthoven: NPRfoundation; [accessed 2008 November 21]. Available from: http://www.perinatreg.nl/.

27. Rubin DB. Inference and missing data. Biometrika. December 1, 1976 1976;63(3):581-592.

28. Donders AR, van der Heijden GJ, Stijnen T, Moons KG. Review: a gentle introduction to imputation of missing values. J Clin Epidemiol. Oct 2006;59(10):1087-1091.

29. Frank E. Harrell J. Regression Modeling Strategies. New York: Springer; 2001.

30. Steyerberg EW. Clinical Prediction Models. New York: Springer; 2009.

31. Hanley JA, McNeil BJ. A method of comparing the areas under receiver operating characteristic curves derived from the same cases. Radiology. Sep 1983;148(3):839-843.

32. Hosmer DW, Lemeshow S. Applied Logistic Regression. New York: John Wiley \& Sons, Inc.; 2000.

33. Steyerberg EW, Harrell FE, Jr., Borsboom GJ, Eijkemans MJ, Vergouwe Y, Habbema JD. Internal validation of predictive models: efficiency of some procedures for logistic regression analysis. J Clin Epidemiol. Aug 2001;54(8):774-781.

34. Harrell Jr FE. Regression Modeling Strategies. New York: Springer; 2001.

35. Greenland S. Quantifying biases in causal models: classical confounding vs colliderstratification bias. Epidemiology. May 2003;14(3):300-306.

36. Smits LJ, Van Kuijk SMJ, Peeters LL, Prins MH, Sep SJ. Index-event bias - a numerical example. J Clin Epidemiol. 66 (2013) 192-196.

37. Steyerberg EW. Clinical Prediction Models. New York: Springer; 2009.

38. Facchinetti F, Marozio L, Frusca T, et al. Maternal thrombophilia and the risk of recurrence of preeclampsia. Am J Obstet Gynecol. Jan 2009;200(1):46 e41-45.

39. Aardenburg R, Spaanderman ME, van Eijndhoven HW, de Leeuw PW, Peeters LL. A low plasma volume in formerly preeclamptic women predisposes to the recurrence of hypertensive complications in the next pregnancy. I Soc Gynecol Investig. Dec 2006;13(8):598-603.

40. Delahaije DH, van Kuijk SM, Dirksen CD, et al. Cost-effectiveness of recurrence risk guided care versus care as usual in women who suffered from early-onset preeclampsia including HELLP syndrome in their previous pregnancy (the PreCare study). BMC Pregnancy Childbirth.10:60 
Chapter 2 


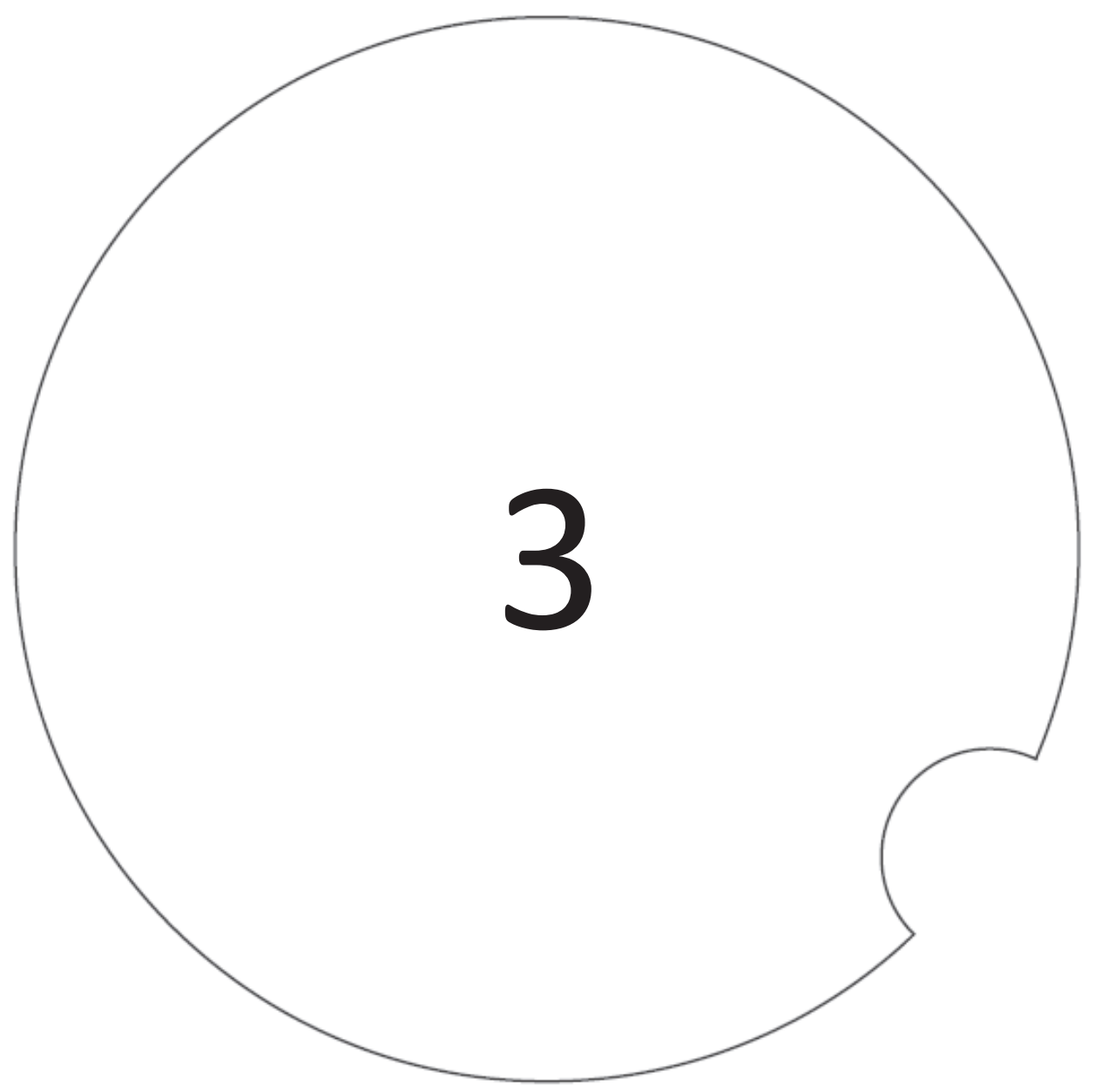

\section{Cost-effectiveness of recurrence risk guided care versus care-as-usual in women who suffered from early-onset preeclampsia in their previous pregnancy}

Delahaije DH, van Kuijk SM, Dirksen CD, Sep SJ, Peeters LL, Spaanderman ME, Bruinse HW, de Wit-Zuurendonk LD, van der Post JA, Duvekot JJ, van Eyck J, van Pampus MG, van der Hoeven MA, Smits LJ BMC Pregnancy and Childbirth 2010, 10:60 


\begin{abstract}
Background

Preeclampsia and HELLP syndrome may have serious consequences for both mother and foetus. Women who have suffered from preeclampsia or the HELLP syndrome have an increased risk of developing preeclampsia in a subsequent pregnancy. However, most women will develop no or only minor complications. In this study, we intend to determine cost-effectiveness of recurrence risk guided care versus care as usual in pregnant women with a history of early-onset preeclampsia.
\end{abstract}

\title{
Methods/design
}

We developed a prediction model to estimate the individual risk of recurrence of earlyonset preeclampsia and the HELLP syndrome. In a before-after study, pregnant women with preeclampsia or HELLP syndrome in their previous pregnancy receiving care as usual (before introduction of the prediction model) will be compared with women receiving recurrence risk guided care (after introduction of the prediction model).

Eligible and pregnant women will be recruited at six university hospitals and seven large non-university tertiary referral hospitals in the Netherlands.

The primary outcome measure is the recurrence of early-onset preeclampsia or HELLP syndrome in women allocated to the regular monitoring group.

For the economic evaluation, a modelling approach will be used. Costs and effects of recurrence risk guided care with those of care as usual will be compared by means of a decision model. Two incremental cost-effectiveness ratios will be calculated: 1) cost per Quality Adjusted Life Year (mother unit of analysis) and 2) cost per live born child (child unit of analysis).

\section{Discussion}

This is, to our knowledge, the first study that evaluates prospectively the efficacy of a multivariable prediction rule for recurrent hypertensive disease in pregnancy. Results of this study could either be integrated into the current guideline on Hypertensive Disorders in Pregnancy, or be used to develop a new guideline. 


\section{Background}

\section{The disease}

Preeclampsia is defined as de novo development of hypertension (>140/90 $\mathrm{mmHg}$ ) in combination with proteinuria after 20 weeks of gestation in pregnant women $\left({ }^{1}\right)$. One of the most severe forms of this disease is called the HELLP syndrome, which is derived from the acronyms of the symptoms (Haemolysis, Elevated Liver enzymes and Low Platelets).

Preeclampsia (also termed early-onset preeclampsia) and HELLP syndrome may be lifethreatening for both mother and child ${ }^{2}$. In the mother, these disorders predispose to premature cardiovascular disorders such as chronic hypertension, ischemic heart disease and stroke later in life $^{3}$. Studies in children, born after preeclamptic pregnancies and who were relatively small at birth, have shown an increased risk of stroke in adult life, an increased risk of coronary heart disease and metabolic syndrome ${ }^{4,5,6}$. Patients and their partners perceive preeclampsia and especially HELLP syndrome as a highly traumatic life event, both during and after pregnancy ${ }^{7}$.

In the United States and Europe, the prevalence of clinically relevant preeclampsia is approximately $2 \%$ of all pregnancies, with HELLP syndrome complicating preeclampsia approximately in 10 to $20 \%$ of the cases ${ }^{3,8}$. Maternal mortality rate in the Netherlands due to hypertensive disease during pregnancy amounts to 4.0 per 100,000 live births in 1993-2002 ${ }^{9}$.

Although several risk factors have been identified, it is difficult to develop effective strategies for the prevention and treatment of these disorders ${ }^{3}$. Strategies applied nowadays are diverse and include antenatal surveillance, modification of lifestyle, dietary interventions and pharmacological therapy. In the last two decades, clinical guidelines for the management of preeclampsia and HELLP syndrome have been adopted in most developed countries ${ }^{10,11,12}$.

Clinical management of preeclampsia or HELLP syndrome depends on the gestational age at onset, severity of symptoms, laboratory abnormalities, size and condition of the unborn infant, disease progression and response to symptomatic treatment.

Mild preeclampsia is usually managed expectantly, as opposed to the management of severe preeclampsia, which is more pro-active consisting of the administration or magnesium sulphate, often combined with antihypertensives ${ }^{13}$. When gestational age is less than 34 weeks and both maternal and foetal condition are satisfactory, it is usually recommended to prolong pregnancy for at least 48 hours to benefit optimally from the enhancing effect of corticosteroid administration on foetal lung maturation. Foetal surveillance is an important component of the management. Delivery is the only causal treatment and therefore, the management of choice from 37 weeks' gestation onward $^{14,15}$.

The health care problem

Although preeclampsia and HELLP syndrome are considered diseases of the first pregnancy, the risk of developing recurrent preeclampsia or HELLP syndrome is 
increased among parous women with preeclampsia and/or HELLP syndrome in their previous pregnancy. Sep et al. ${ }^{16}$ conducted a literature search in order to identify prediction tests for recurrent disease. The recurrence rates reported vary from none to 31 percent for preeclampsia and from 3 percent to 7 percent for HELLP syndrome. Fortunately, the majority of women with a history of preeclampsia or HELLP syndrome have uncomplicated subsequent pregnancies.

To the best of our knowledge there is at this moment no consensus about the management of pregnant women with a history of early-onset preeclampsia or HELLP syndrome.

As a result, follow-up and counselling of these patients varies per centre, gynaecologist and patient, and is largely based on the perceived risk by the responsible care provider of recurrence of the disease in the next pregnancy and the level of anxiety of the patient and its resulting demand for care. Formerly preeclamptic patients are often subjected to various medical screening programmes to detect associated disorders, followed by additional exams by other specialists or the initiation of some management (postpartum evaluation). During a next pregnancy, care varies from regular surveillance by the gynaecologist to intensive surveillance and counselling in order to identify the onset of adverse pregnancy course as early as possible.

\section{Motivation and relevance for the study}

This lack of uniformity in the treatment of these patients asks for more standardisation, e.g. by providing care tailored to the individualised risk assessment.

Current policy may not be efficient. Since only a small percentage of these women develop early-onset recurrent disease in their next pregnancy, clinical management in the next pregnancy may benefit from subdividing these women into subgroups with or without increased risk. The care provided to these women can then be adjusted to their actual risk profile. Particularly former patients at low risk are expected to benefit from this type of risk stratification. Recurrence risk guided care could lead to a substantial reduction of (health care) costs and an increased quality of care.

We have recently developed a multiple-factor model for the prediction of recurrent early-onset preeclampsia and/or HELLP syndrome during the current pregnancy in women with a prior pregnancy complicated by preeclampsia and/or HELLP syndrome $^{17}$.

The PreCare study (pregnant women with previous preeclampsia: efficiency of care based on recurrence risk estimation), was designed to (1) estimate cost-effectiveness of recurrence risk guided care versus care as usual for pregnant women with a previous pregnancy being complicated by preeclampsia or HELLP syndrome and (2) to validate the prediction model externally on the basis of the results of a prospective cohort and to update the prediction model if necessary. To this end, we will use the prediction model to differentiate the intensity of monitoring of pregnant women with a history of early-onset preeclampsia and/or HELLP syndrome. Patients are assigned to either the 'regular monitoring' or 'intensive monitoring' protocol. We will compare this 
strategy, referred to as recurrence risk guided care, with usual care for these patients in The Netherlands.

For this purpose, the following research questions were specified:

1. What are the effects of recurrence risk guided care versus care as usual for pregnant women with a previous preeclamptic pregnancy, on maternal and foetal morbidity and mortality?

2. What are the effects of recurrence risk guided care versus care as usual on specific and generic quality of life, anxiety, depression and development of posttraumatic stress?

3. What are the societal costs associated with the effects of recurrence risk guided care versus care as usual?

4. What is the cost-effectiveness of recurrence risk guided care versus care as usual?

\section{Development of prediction model}

Although earlier studies have identified individual predictive factors for recurrent preeclampsia, a combination of variables for the prediction of recurrent preeclampsia have not been explored until recently. A simple pre-pregnant prediction rule which includes several predictive factors was developed by Sep et al. ${ }^{18}$. Unfortunately, patient data were collected from a single hospital and the number of included patients was limited $(n=186)$.

We developed a prediction model to estimate the individual recurrence risk of recurrence of early-onset preeclampsia and/or the HELLP syndrome. We used data of 407 women with early-onset preeclampsia and/or the HELLP syndrome in their first pregnancy, who had undergone subsequent postpartum screening and who had a recorded consecutive ongoing pregnancy for which maternal and neonatal outcomes were available. Data were collected from four university hospitals (Maastricht University Medical Centre, University Medical Centre Utrecht, Academic Medical Centre Amsterdam and University Medical Centre St. Radboud Nijmegen), and one tertiary referral hospital (Máxima Medical Centre Veldhoven).

Predictors of recurrent disease were preselected based on availability in the five different hospitals, previous literature and gynaecologists' expert opinion. Fasting circulating level of glucose measured at postpartum screening, gestational age at delivery of the first pregnancy, prior small-for-gestational-age newborn, chronic hypertension and maternal BMI before the second pregnancy proved to be the predictors within a logistic regression mode ${ }^{17}$.

Since a model tends to perform best in the derivation sample, called 'overfitting', we used bootstrapping techniques to internally validate the model. A shrinkage factor was computed to shrink the regression coefficients in order to get a more conservative risk estimate. 


\section{Methods/design}

\section{Design}

The PreCare study uses a before-after design, in which outcomes and costs before the introduction of the prediction model (i.e. care as usual) are compared with outcomes and costs after introduction of the prediction model (i.e. recurrence risk guided care).

This study consists of two consecutive phases. In the first phase of the study, 50 women receiving care as usual will be followed prospectively from their first pregnancy-related outpatient visit until 3 months postpartum. These 50 women will receive questionnaires in order to measure quality of life, anxiety, depression, posttraumatic stress and costs outside the hospital. Retrospective data of an additional group of 200 women who have been treated with care as usual in the past will be collected later on. Then the prediction rule will be introduced. In the second phase, 250 women will receive either protocolised regular monitoring or protocolised intensive monitoring depending on their risk of developing early-onset preeclampsia or HELLP syndrome during their current pregnancy, as estimated on the basis of the prediction model. In regular monitoring, pregnant women are monitored less intensively than in intensive monitoring. Regular monitoring and intensive monitoring are described in detail in the 'Monitoring protocols' section. Figure 1 summarises the design.

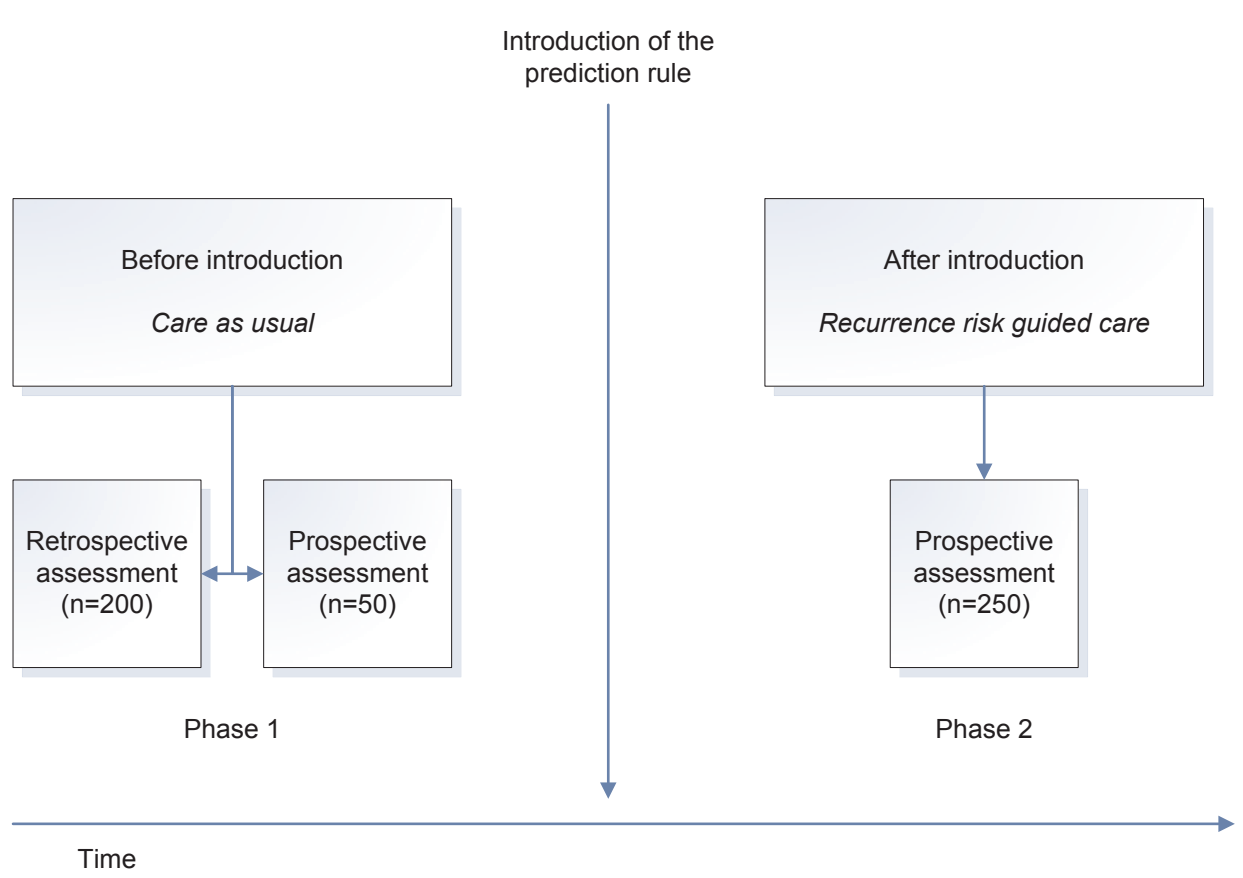

Figure 1: Design of the study 


\section{Participants/eligibility criteria}

In phase 1 and 2, pregnant women (aged 18 years and older) with preeclampsia and/or HELLP syndrome in their previous pregnancy who have visited the outpatient clinic until $16+6$ weeks of pregnancy are eligible for participation in the PreCare study.

Given these general eligibility criteria, two subgroups are distinguished in recurrence risk guided care (phase 2 ).

In women who gave birth after early-onset preeclampsia or HELLP syndrome until 33+6 weeks gestational age in the previous pregnancy, the prediction model is applied in order to allocate the participants to one of the two monitoring protocols. The data, which are required as input parameters for the prediction model, should be available. These variables mostly come from the postpartum evaluation.

Women who gave birth between 34 and 36+6 weeks gestational age in the previous pregnancy face a relatively small risk of developing recurrent preeclampsia or HELLP syndrome, compared to women who gave birth before 34 weeks. Therefore, they all are allocated to the regular monitoring subgroup ${ }^{19}$.

Women suffering from severe co-morbidity, such as diabetes mellitus, SLE, renal disease, cardiac disease or anti-phospholipids syndrome are excluded from the study as they will all receive (or have received where it concerns the retrospective patients) intensive surveillance. The current study will not include women who gave birth after 37 weeks gestational age.

The study population is recruited from six university hospitals and seven large nonuniversity tertiary referral hospitals in the Netherlands: the Maastricht University Medical Centre, University Medical Centre St. Radboud Nijmegen, the Isala Clinics Zwolle, Erasmus Medical Centre Rotterdam, Academic Medical Centre Amsterdam, University Medical Centre Utrecht, Máxima Medical Centre Veldhoven, the University Medical Centre Groningen, Martini Hospital Groningen, Amphia Hospital Breda, Atrium Medical Centre Heerlen, Jeroen Bosch Hospital Den Bosch and Kennemer Hospital Haarlem.

\section{Recruitment procedures}

For the prospective assessment, 50 care as usual patients and 250 recurrence-risk guided patients will be included in the study.

The research nurse or the gynaecologist of participating hospitals identifies eligible women by screening the appointment system of the outpatient clinic. After the pregnant woman has given informed consent for participation in the study, patients in phase 1 will be enrolled in care as usual whereas patients in phase 2 will be enrolled in recurrence risk guided care.

In recurrence risk guided care (phase 2), a simple web based risk calculator for the determination of recurrence risk and required intensity of surveillance has been developed and has been made accessible to gynaecologists and research nurses through the Internet. 
Information about the recommended components of intensive monitoring and regular monitoring has been provided together with the risk calculator.

For the retrospective assessment, 200 patients who were treated according to care as usual will be used as a comparator group based on matching. Matching criteria will at least include maternal age (difference $<5$ years) and gestational age at delivery. Consequently, at study completion, there will be 250 care-as-usual patients who will be compared with 250 recurrence risk guided patients.

\section{Data collection}

For the prospective assessment ( $n=50$ care as usual; $n=250$ recurrence risk guided care), baseline demographics, past obstetric and medical history will be recorded in case report forms. Information is obtained on the condition of mother and child, infant weight, infant length, morbidity and mortality from the infant and maternal records. If applicable, details of the admittance of the child to the neonatal intensive care, high care or medium care unit will also be collected. All activities in the hospital will be documented until three months postpartum.

We will assess not only clinical outcomes, but also health-related quality of life by using the EQ- $5 \mathrm{D}^{20}$, anxiety by using the STAI ${ }^{21}$, depression by using the Beck Depression Inventory ${ }^{22}$ and posttraumatic stress by using the PTSD Symptom Scale ${ }^{23}$. The QoL questionnaires will be administered at 3-month intervals between baseline (before 20 weeks of pregnancy), at 29 weeks of pregnancy, ten days and three months postpartum. Costs outside the hospital, such as GP care, midwife and maternity care, productivity losses and out-of-pocket expenses will be obtained by means of a retrospective cost questionnaire with a recall period of 3 months. Long-term follow up of the women and children may be possible, but is not included in the current study.

For women enrolled in the retrospective assessment $(n=200)$, all data described above will be collected, except data on health-related quality of life, anxiety, depression, posttraumatic stress and costs outside the hospital.

\section{Monitoring protocols}

\section{Development of the protocols}

All gynaecologists of the participating centres were approached and they participated in a consensus process, during which the final protocols were designed. Nearly all were obstetricians specialised in hypertensive disorders of pregnancy. After reaching consensus, the protocols were distributed among all providers of care in the study.

\section{Regular monitoring}

Women will visit the outpatient clinic 9 - 11 times: (8, 12), 16, 20, 24, 28, 32, 36, 38, 39 and 40 weeks gestational age. The visits at 16 and 20 weeks will be scheduled after the first trimester screening procedure and the routine ultrasound screening for structural malformations, respectively. If feasible, six of these eleven visits $(12,20,28,36,39,40)$ ought to be performed by the same obstetrician, whereas the remaining five 
(reassuring) visits $(8,16,24,32,38)$ may be performed by a nurse-practitioner or may be performed by the gynaecologist of the referring clinic (as of 32 weeks, in regular and intensive monitoring).

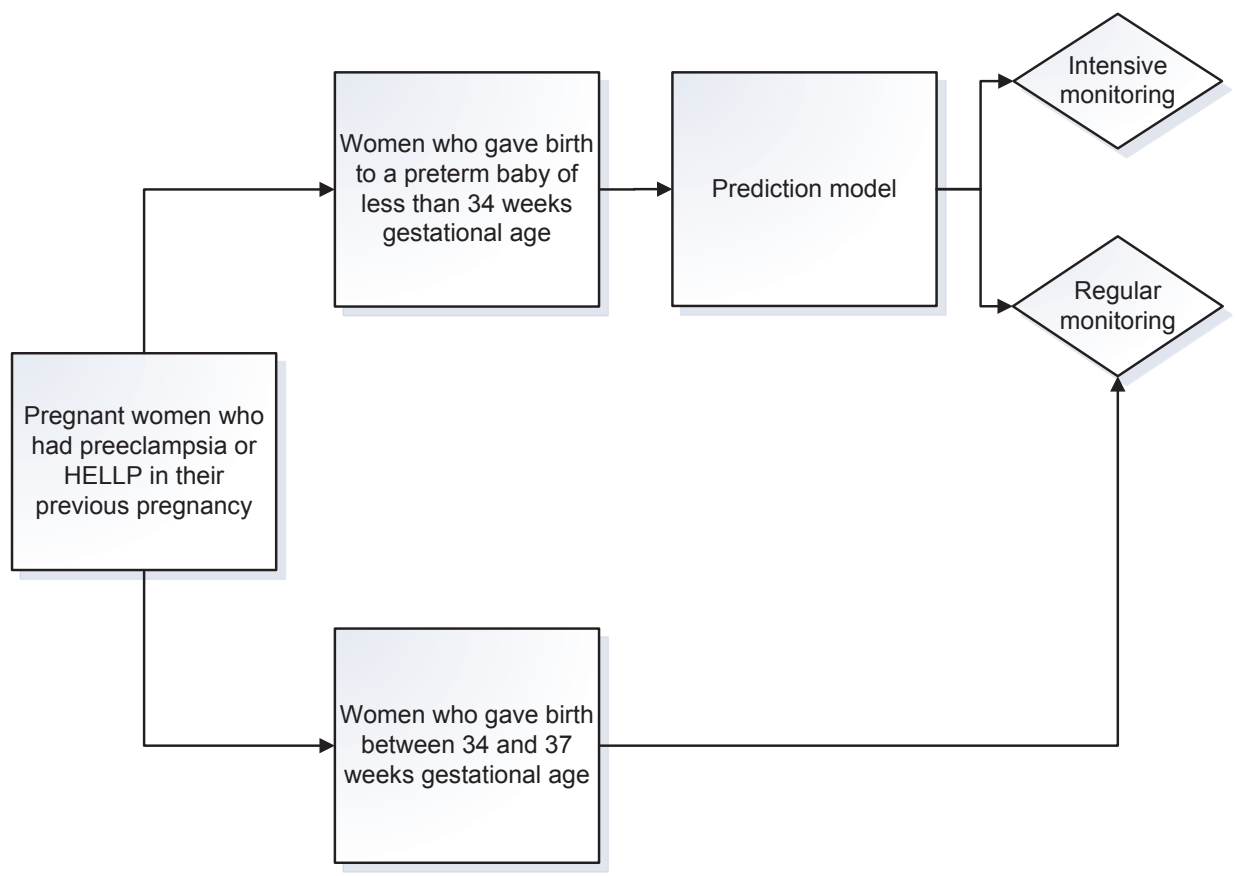

Figure 2: Recurrence risk guided care (phase 2)

\section{Intensive monitoring}

Women will visit the outpatient clinic $14-16$ times: $(8,12), 16,18,20,22,24,26,28$, $30,32,34,36,38,39$ and 40 weeks gestational age.

At two occasions during an intensive monitoring pregnancy (16 and 20 weeks), the patient will undergo a series of diagnostic tests, which consist of

1. Measurements in urine: (micro)albuminuria, creatinine, protein (protein serves to calculate the protein-to-creatinine ratio)

2. Measurements in blood: Haemoglobin, platelet count, mean platelet volume, creatinine, urate, CRP, glucose, fibronectin, Flt-1, endoglin

3. $24 \mathrm{~h}$ blood pressure monitoring. This assessment is optional (not obligatory)

4. Assessment of the Doppler flow velocity profile in both uterine arteries (at 20 weeks)

Activities other than, or additional to those described in the regular monitoring and intensive monitoring protocols (such as intercurrent admissions, additional outpatient visits or diagnostic testing, or telephone/e-mail contacts) will be registered. 


\section{Study outcomes}

Recurrence risk guided care will be compared with care as usual with respect to several outcomes.

Primary outcome is the occurrence of either early-onset preeclampsia or HELLP syndrome. Early-onset preeclampsia and early-onset HELLP syndrome are defined according to the criteria of the International Society for the Study of Hypertension in Pregnancy with the criterion of delivery before 34 weeks after the last menstrual period $^{24}$. Secondary clinical outcome measures are: gestational age of onset of preeclampsia, eclampsia, HELLP syndrome, intrauterine growth restriction, caesarean section, admission to the neonatal intensive care unit (NICU), gestational age at delivery and maternal/infant mortality. Other secondary outcome measures are societal costs, quality of life, anxiety, depression, and development of posttraumatic stress, satisfaction with treatment, protocol adherence and cost-effectiveness.

\section{Statistical analysis}

Sample size

Sample size calculation was based on the expected fraction of women assigned to regular monitoring either on the basis of the prediction rule or gestational age at the time of previous delivery (67\%), the expected failure rate within this category (2\%), a type I error (one-sided) of 0.05 and a type II error of 0.2. In order to be able to exclude failure rates of $5 \%$ or more, 150 patients assigned to regular monitoring are needed. This means that results of about 225 patients in the recurrence risk based care are needed. The estimated number of 250 allows for drop out and incomplete data of 25 patients.

\section{Data analysis}

The primary analysis concerns the incidence of recurrent early-onset preeclampsia or HELLP syndrome in both groups (recurrence risk guided care versus care as usual). Analyses will be adjusted for potential confounders (including demographic factors). Confidence intervals will be adjusted by means of multilevel analysis (hospital).

We will also analyse whether the new patient data indicate the need for an update of the predictive model. For this purpose, we will first compare the characteristics of the original population used for model development with the new population, the socalled "validation population". For example, the incidence of early-onset preeclampsia may differ between the original and validation population. It is also possible that the latter population has a different case-mix (i.e. differences in distributions of the predictors in the population), because, e.g., more hospitals are included. In addition, different predictor-outcome associations and additional predictors, that are not included in the model but are either more or less frequent in the new population, could play a role. We will also assess model performance in the validation population by comparing sensitivity, specificity, positive and negative predictive values and discrimination and calibration compared to the performance in the derivation population. 
If the analysis should indicate lower accuracy than expected, we will analyse to what extent all these factors indeed affect the model's accuracy and update the model to obtain adequate accuracy. Updating of the model will be done by means of recalibration (step 1) and model revision (step 2$)^{25,26,27}$.

\section{Economic evaluation}

A cost-effectiveness analysis will be performed from a societal perspective, comparing the costs and effects of recurrence risk guided care versus care as usual. The time horizon of the study is 9 months ( 3 months amenorrhea - 3 months postpartum). Discounting is not relevant given the short time horizon.

The cost analysis will be performed according to the Dutch guidelines for cost calculations ${ }^{28}$. All hospital resource use and costs associated with care for pregnant women and their newborns will be calculated from study entry until 3 months postpartum and include costs such as outpatient visits and hospital admissions. Cost prices will be obtained from participating hospitals. If prices are not readily available, directive prices will be used ${ }^{28}$ or additional calculations will be made. Costs in the analysis also include direct non-health care costs (travel costs) and indirect costs (productivity loss).

Effect parameters are clinical outcomes, health related quality of life, anxiety, depression and posttraumatic stress.

Currently, no economic evaluation methods are available that integrate health outcomes of both mother and child into a single outcome measure. However, preeclampsia may have health effects for both the pregnant women and her (unborn) child. Therefore, the main cost-effectiveness analysis will involve calculating two incremental cost-effectiveness ratios (ICERs) expressing 1) the cost per Quality Adjusted Life Year (QALY, mother unit of analysis) and 2) the cost per live born infant (child unit of analysis). To this end, a decision model will be developed. Modelling in economic evaluation is considered useful for example when experimental observations from a trial are missing, which in this study applies to some parameters in the care as usual group ${ }^{29,30}$.

\section{Ethical considerations}

This study has been approved by the ethical committee of the University Hospital Maastricht (Ref.no. MEC 07-2-078). A total of six academic and seven non-academic hospitals participate in the study; all of them have completed their obligatory feasibility assessment procedure successfully. Informed consent is being obtained from all patients prior to enrolment into the study.

\section{Discussion}

This study is expected to yield information on health outcomes and costs of adjusting the level of care to the estimated probability of recurrent preeclampsia. The results can provide a basis for more uniform (and evidence-based) guidelines for care for 
formerly preeclamptic women and possibly lead to more cost-effective provision of health care. With respect to health care costs, it is expected that mean costs per patient will decrease as a result of a reduction in intensive maternal and foetal surveillance. Potential savings can be even higher, since the majority of women assigned to regular monitoring may be adequately served by care provided by inhospital midwifes ${ }^{31}$.

The close cooperation with many centres enables us to reach a representative study population of pregnant women who have experienced preeclampsia in their previous pregnancy, which enhances the applicability of the results to all former preeclamptic women. Results of this study will be disseminated by means of presentations at scientific meetings and peer-reviewed publications. Study outcomes will also be communicated directly to the NVOG (Dutch Association of Obstetrics and Gynaecology), KNOV (Royal Dutch Association of Midwives), and the Dutch HELLP syndrome foundation. In addition, we will cooperate with the NVOG in order to produce recommendations for the formulation of guidelines. The recommendations could either be integrated into the current guideline on Hypertensive Disorders in Pregnancy, or be used to develop a new guideline. The results of this study will be used to standardise the postpartum evaluation of women with a recent history of preeclampsia or HELLP syndrome. It is expected that the number of tests in the postpartum evaluation will not only be reduced, but also synchronised.

A randomised controlled trial design is usually preferred over any other design. However, for this study such a design was not considered applicable. A before-after study was chosen (instead of a fully prospective, randomised study) because of the risk of care as usual being contaminated with the regular monitoring and intensive monitoring protocols. Because current care as usual is not standardised, and blinding of the participating gynaecologists with respect to predictive factors for recurrent disease is practically unfeasible, a randomised design could reduce the contrast between study arms and thereby threaten the validity of the results.

As the care provided to women assigned to regular monitoring is less intense, a possible consequence may be that the detection of clinical signs of preeclampsia or HELLP syndrome is somewhat later than in the current care as usual approach. However, we do not expect this to lead to adverse maternal or foetal outcomes, because the prediction model suggests that the recurrence rate in this group is low $<$ $1 \%)$ whereas there is also increased alertness for early signs of pregnancy complications in women with a history of preeclampsia or HELLP syndrome.

In summary, the PreCare study is designed to provide information on whether recurrence risk guided care is a worthwhile strategy compared to current care for pregnant women who suffered from preeclampsia or HELLP syndrome during their previous pregnancy. 


\section{References}

1. Davison JM, Homuth V, Jeyabalan A, Conrad KP, Karumanchi A, Quaggin S, Dechend R, Luft FC: New aspects in the pathophysiology of preeclampsia. J Am Soc Nephrol 2004, 15:24402448.

2. Barden A: Pre-eclampsia: contribution of maternal constitutional factors and the consequences for cardiovascular health. Clin Exp Pharmacol Physiol 2006, 33:826-830.

3. Meads CA, Cnossen JS, Meher S, Juarez-Garcia A, ter Riet G, Duley L, Roberts TE, Mol BW, van der Post JA, Leeflang MM, Barton PM, Hyde CJ, Gupta JK, Khan KS: Methods of prediction and prevention of pre-eclampsia: systematic reviews of accuracy and effectiveness literature with economic modelling. Health Technol Assess 2008, 12:1-270.

4. Osmond C, Kajantie E, Forsén TJ, Eriksson JG, Barker DJ: Infant growth and stroke in adult life: the Helsinke Birth Cohort Study. Stroke 2007, 38:264-270.

5. Eriksson JG, Forsén T, Tuomilheto J, Osmond C, Barker DJ: Early growth and coronary heart disease in later life: longitudinal study. BMJ 2001, 322:949-953.

6. Barker DJ, Martyn CN, Osmond C, Hales CN, Fall CH: Growth in utero and serum cholesterol concentrations in adult life. BMJ 1993, 307:1524-1527.

7. Sibai B, Dekker G, Kupferminc M: Pre-eclampsia. Lancet 2005, 365:785-799.

8. Haram K, Svendsen E, Abildgaard U: The HELLP syndrome: Clinical issues and management. A Review. BMC Pregnancy Childbirth 2009, 9:8.

9. Schutte JM, Schuitemaker NWE, van Roosmalen J, Steegers EAP: Substandard care in maternal mortality due to hypertensive disease in pregnancy in the Netherlands. BJOG 2008, 115:732-736.

10. NVOG (2005). Richtlijn 6 - Hypertensieve aandoeningen in de zwangerschap; mei 2005 [http://www.nvog.nl/files/rl_06_hypertensieve_aandoeningen_in_de_zwangerschap_herzien.pdf]

11. Society of Obstetricians and Gynaecologists of Canada: Diagnosis, evaluation and management of the hypertensive disorders of pregnancy. J Obstet Gynaecol Can 2008, 30 (Suppl 1).

12. Société française d'anesthésie et de réanimation (Sfar), Collège national des gynécologues et obstétriciens français (CNGOF), Société française de médecine périnatale (SFMP) and Société française de néonatalogie (SFNN): Multidisciplinary management of severe preeclampsia. Experts' guidelines 2008. Ann Fr Anesth Reanim 2009, 28:275-281.

13. Berkow, R., Beers, M.H., Fletscher, A.J. (2000). Merck Manual Medisch handboek. Bohn Stafleu Van Loghum, Houten/Diegem.

14. Williams J, Mozurkewich E, Chilimgras J, van de Ven C: Critical care in obstetrics: pregnancy-specific conditions. Best Pract Res Clin Obstet Gynaecol 2008, 22:825-846.

15. Koopmans CM, Bijlenga D, Groen H, Vijgen SM, Aarnoudse JG, Bekedam DJ, van den Berg PP, de Boer K, Burggraaf JM, Bloemenkamp KW, Drogtrop AP, Franx A, de Groot CJ, Huisjes AJ, Kwee A, van Loon AJ, Lub A, Papatsonis DN, van der Post JA, Roumen FJ, Scheepers HC, Willekes C, Mol BW, van Pampus MG: Induction of labour versus expectant monitoring for gestational hypertension or mild pre-eclampsia after 36 weeks' gestation (HYPITAT): a multicentre, open-label randomised controlled trial. Lancet 2009, 374:979-988.

16. Sep S, Smits L, Prins M, Peeters L: Prediction tests for recurrent hypertensive disease in pregnancy, a systematic review. Hypertens Pregnancy 2010, 29:206-230.

17. van Kuijk SMJ, Nijdam, ME, Janssen KJ, Sep SJ, Peeters LL, Delahaije DHJ, Spaanderman ME, Bruinse HW, Franx A, van Rijn BB, Bots ML, Langenveld J, van der Post AM, Smits LJ: A preconceptional prediction model for recurrent early-onset preeclampsia and the HELLP syndrome. Reprod. Sci. 2011 Nov;18(11):1154-9. 
18. Sep SJS, Smits LJM, Prins MH, Spaanderman MEA, Peeters LLH: Simple prepregnant prediction rule for recurrent early-onset hypertensive disease in pregnancy. Reprod Sci 2009, 16:80-87

19. Zhang J, Troendle JF, Levine RJ: Risks of hypertensive disorders in the second pregnancy. Paediatr Perinat Epidemiol 2001, 15:226-231

20. Anonymus: EuroQoL-a new facility for the measurement of health-related quality of life. The EuroQol Group. Health Policy 1990, 16:199-208.

21. Spielberger CD, Gorsuch RL, Lushene RE (1970). STAI Manual for the State-trait Anxiety Inventory. Consulting Psychologists Press: Palo Alto, CA.

22. Beck, AT, Erbaugy JK, Mock JE, Mondelson M, Ward CH: An inventory for measuring depression. Arch Gen Psychiatry 1961, 4:561-671.

23. Foa EB, Riggs DS, Rothbaum B: Reliability and validity of a brief instrument for assessing post traumatic stress disorder. JTS 1993, 6:459-473.

24. Brown MA, Lindheimer MD, de Swiet M, van Assche A, Moutquin JM: The classification and diagnosis of the hypertensive disorders of pregnancy: statement from the International Society for the Study of Hypertension in Pregnancy (ISSHP). Hypertens Pregnancy 2001, 20:IX-XIV

25. van Houwelingen JC, Thorogood J: Construction, validation and updating of a prognostic model for kidney graft survival. Stat Med 1995, 14:1999-2008.

26. van Houwelingen JC: Validation, calibration, revision and combination of prognostic survival models. Stat Med 2000, 19:3401-15.

27. D'Agostino RB, Grundy S, Sullivan LM, Wilson P: Validation of the Framingham coronary heart disease prediction scores: results of a multiple ethnic groups investigation. JAMA 2001. 286:180-7.

28. Oostenbrink JB, Bouwmans CAM, Koopmanschap MA, Rutten FFH: Handleiding voor kostenonderzoek, methoden en standaard kostprijzen voor economische evaluaties in de gezondheidszorg. Amstelveen: College voor Zorgverzekeringen (CVZ); 2004.

29. Buxton MJ, Drummond MF, van Hout BA, Prince RL, Sheldon TA, Szucs T, Vray M: Modelling in economic evaluation: an unavoidable fact of life. Health Econ 1997, 6:217227.

30. Brennan A, Akehurst R: Modelling in Health Economic Evaluation: What is its Place? What is its Value? Pharmacoeconomics 2000, 17:445-459.

31. PRN/TNO (2002). De thuisbevalling in Nederland, 1995-2002. Rapportage over de jaren 2001-2002 van PRN/TNO. [http://www.perinatreg.nl/uploads/153/99/Rapportage_Thuisbevalling_2001-2002.pdf]

32. ZonMW projectenpoort - Cost-effectiveness of recurrence risk-guided care of pregnant women with preeclampsia in the previous pregnancy. [http://www.zonmw.nl/nl/system/ zoekresultaten/delfi/projecten-database/projectdetail/?tx_videlfiprojecten_pi1\%5Bproject_id\%5D=4600267139 


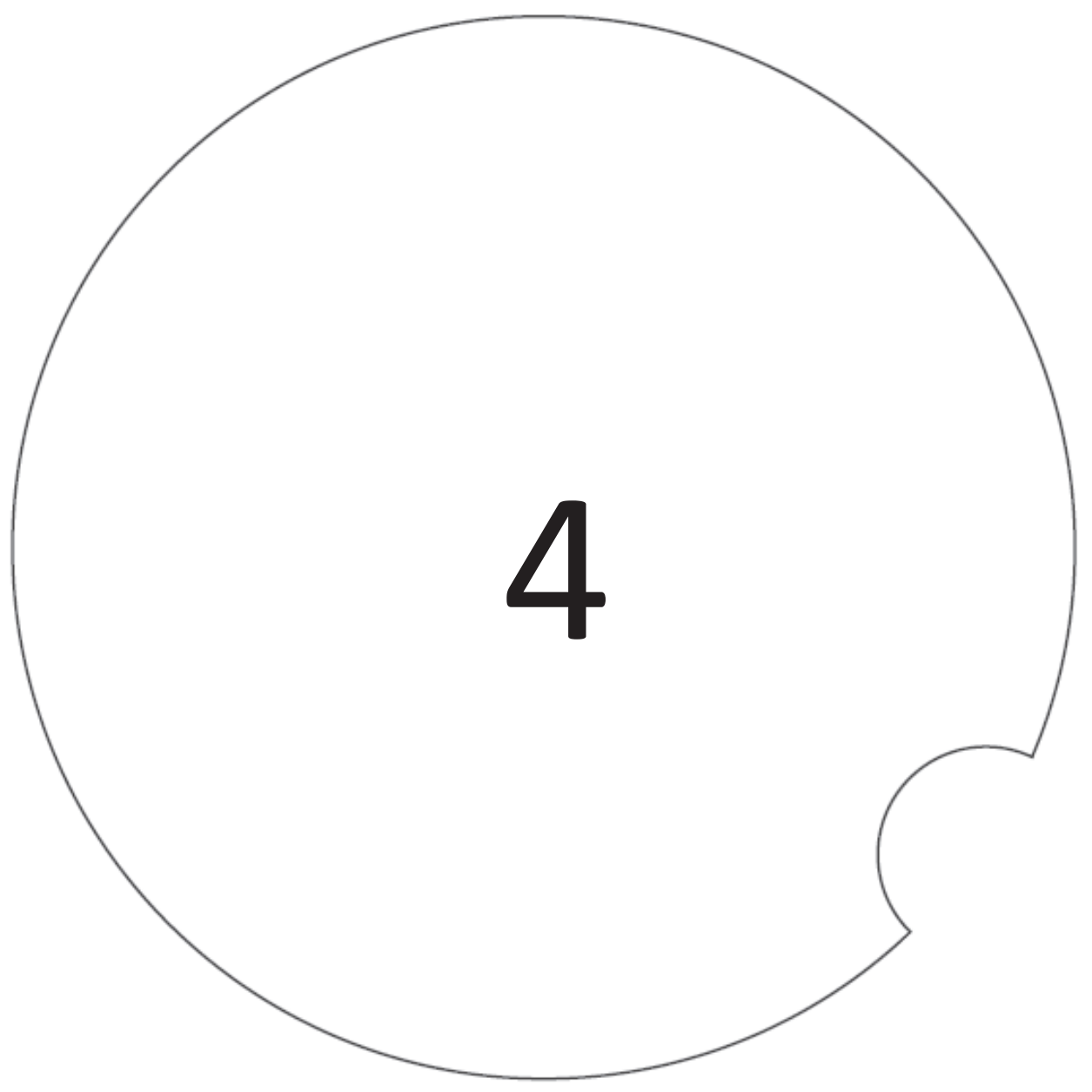

\section{External validation of a model for preconceptional prediction of recurrent early-onset preeclampsia}

van Kuijk SM, Delahaije DH, Dirksen CD, Scheepers HC, Spaanderman ME, Ganzevoort W, Duvekot JJ, Oudijk MA, van Pampus MG, von Dadelszen P, Peeters LL, and Smits LJ Submitted 


\begin{abstract}
Introduction

In an earlier paper we reported on the development of a prediction model aimed at the identification of women at low risk of recurrence based on data of 407 women from 5 Dutch hospitals.
\end{abstract}

\title{
Objective
}

To validate and recalibrate a prediction model for the risk of recurrent early-onset preeclampsia in former patients.

\section{Methods}

We collected both prospective and retrospective data about course and outcome of the next ongoing pregnancy in 229 women with a history of early-onset preeclampsia. Recurrence was defined as preeclampsia requiring delivery for medical reasons before 34 weeks of gestation. We computed their individual risk using a model published previously, and assessed performance of this model by computing measures of discrimination and calibration.

\section{Results}

Early-onset preeclampsia recurred in $6.6 \%$ of former patients. The model systematically underestimated recurrence risk, indicating poor calibration. The model's discriminative ability was modest as indicated by the area under the receiver operating characteristic curve (AUC) of 59\% (95\% Cl: $45-73)$. For a number of relevant riskthresholds, the model created groups that were only moderately different in terms of their average risk of recurrent early-onset preeclampsia.

\section{Conclusion}

The model's discriminate ability, as indicated by the AUC, was poor. Its predictive performance was insufficient to classify former patients into clinically relevant risk groups. 


\section{Introduction}

It is common practice in the Netherlands that apparently healthy women with a history of early-onset preeclampsia, defined as preeclampsia (PE) requiring delivery before 34 weeks gestation, are counselled before they will conceive a next pregnancy. During this counselling visit, and in line with international recommendations ${ }^{1,2}$, they are often screened for subclinical disorders known to predispose to recurrence of hypertensive pregnancy disorders. In their next pregnancy, usually irrespective of screening outcome, they will receive intensive antenatal care consisting of frequent antenatal check-ups, extra ultrasound exams and biochemical testing. Although evidence for a lower recurrence rate of hypertensive complications after intensified antenatal care is lacking, this approach is generally believed to enhance early detection. The overall recurrence rate of early-onset PE in apparently healthy former patients is only about $7 \% \%^{3,4}$. Identification of women at low risk may help decreasing the burden of intensified surveillance for some patients, and reducing healthcare costs in general.

Previously, we presented a newly developed prediction model aimed at the identification of women at low risk of early-onset recurrence, based on data of 407 women from 5 Dutch hospitals ${ }^{5}$. The prediction model estimates the individual risk of recurrence, based on 5 easily available variables obtained in the interval between the complicated and the next ongoing pregnancy (fasting glucose level, body mass index (BMI), duration of the previous complicated pregnancy, the birth of a small-forgestational-age (SGA) infant, and presence or absence of chronic hypertension). After adjusting regression coefficients and intercept in an internal validation step, the model's discriminative ability, quantified as the area under the receiver operating characteristic (ROC) curve, was $65 \%(95 \% \mathrm{Cl}$ : $56-74)$ with good calibration. Analysis of different risk cut-off values showed this model could be used to identify women at low-risk of early-onset recurrence.

In prediction modelling research, a model usually performs better in the cohort used for its development, than in a newly assembled, but comparable, validation cohort ${ }^{6}$. Therefore, introduction of a model with adequate internal validity in the clinic should be preceded by experimental confirmation of its external validity ${ }^{7,8}$. The model's performance in such a new cohort is likely to be comparable to that in future patients from comparable populations as the one used for the external validation.

This study aims to determine the external validity of our previously reported prediction model $^{5}$. To this end, we quantified its discriminative ability and calibration measures, and determined its clinical applicability for a number of different risk thresholds. We also assessed the model's discriminative ability in predicting late-onset PE, all forms of $\mathrm{PE}$, and maternal placental syndrome. 


\section{Methods}

\section{Patients}

We conducted a combined retrospective and prospective cohort study in 12 hospitals throughout the Netherlands between August 2008 and May 2012. For the prospective part of the study, women were identified by screening the appointment system of the outpatient clinic, and checked for eligibility by the research nurses and gynaecologists of the participating hospitals. Eligible pregnant women had a gestational age of less than 17 weeks, and had their preceding pregnancy complicated by early-onset PE and/or the syndrome of haemolysis, elevated liver enzymes and low platelet count (HELLP). Informed consent was obtained for all participants. We defined early-onset as requiring preterm delivery for medical reasons before 34 weeks. We excluded women from enrolment who were younger than 18 years, or had one of the following comorbidities: diabetes mellitus, systemic lupus erythematosus, antiphospholipid syndrome, heart disease or kidney disease. Additionally, we retrospectively collected data from women who had an ongoing pregnancy after a prior pregnancy complicated by $\mathrm{PE}$, applying the same exclusion criteria. Both the prospective group and the retrospective group are part of a project in which we explored the cost-effectiveness of recurrence risk-guided care versus care as usual in women with a history of $P E$, the "PreCare study" ${ }^{9}$. Ethical approval for this study was obtained from the Medical Ethical Committee in Maastricht, the Netherlands (Ref.no. MEC 07-2-078).

In all hospitals we used the same case definition as in the study in which performed to develop the model. $\mathrm{PE}$ was defined as de novo hypertension (systolic blood pressure $(B P) \geq 140 \mathrm{mmHg}$ and/ or diastolic $\mathrm{BP} \geq 90 \mathrm{mmHg}$ ) after 20 weeks' gestation along with de novo proteinuria ( $\geq 300 \mathrm{mg}$ urinary protein excretion/day) ${ }^{10}$. PE superimposed on chronic hypertension was defined as de novo proteinuria complicating pre-existent hypertension after 20 weeks of gestation or a sudden increase in proteinuria or BP in women whose hypertension had previously been well-controlled ${ }^{10}$. We defined HELLP as a pregnancy disorder characterized by thrombocytopenia, haemolysis and abnormal liver enzymes, as specified previously ${ }^{11}$. Recurrence was defined as PE, PE superimposed on chronic hypertension, and/or HELLP requiring delivery for medical reasons before 34 weeks of gestation in the next ongoing pregnancy. We defined maternal placental syndrome as placental dysfunction resulting in any hypertensive disorder of pregnancy, placental abruption or foetal growth restriction ${ }^{12,13}$.

\section{Predictors of the original model}

The original prediction model is shown in box 1 . Circulating levels of glucose were measured after a fasting period of at least 12 hours. If a recent glucose measurement was unavailable, we used the routinely measured early-pregnancy ( $<17$ weeks) screening value instead. We defined hypertension on the basis of a raised BP (systolic BP $\geq 140 \mathrm{mmHg}$ and/ or diastolic BP $\geq 90 \mathrm{mmHg}$ ) at booking, the use of antihypertensive medication, or because hypertension had been diagnosed previously and formally registered in the patient's hospital file. A newborn was considered smallfor-gestational-age when his/her birth-weight was below the $10^{\text {th }} \%$ for gestational age at delivery, based on the most recent Dutch population charts ${ }^{14}$. 


\section{Data analysis}

For analysis of baseline measurements, we used the original values only, without imputation of missing values. Continuous variables are presented as means \pm standard deviation (SD), whereas categorical variables are given as absolute value and percentage.

Some patients had missing predictor variables. We used regression imputation to calculate substitutes for these missing values ${ }^{15}$. We calculated the individual recurrence risk for all subjects using the prediction model formula shown in Box 1. To quantify model performance in the validation cohort, we computed measures of calibration and discrimination. We assessed the model's calibration (i.e. the agreement between observed recurrence rate and predicted risk) by computing calibration-in-thelarge and by visually inspecting the calibration plot. Calibration-in-the-large is a comparison of the mean predicted risk and actual proportion of recurrences, and can provide insight in whether predictions are systematically deviating ${ }^{16}$. In such a scenario, the model's intercept can be adjusted to recalibrate the model. This is performed by computing the intercept necessary for the model's average predicted risk to be equal to the observed recurrence rate, and compute a weighted average between that intercept and the intercept of the initial prediction model. The calibration plot compares predicted risks and observed frequencies in patient groups with similar risk. Ideally, it should follow a 45-degree line. Furthermore, we tested for overall fit using the $\mathrm{H}-\mathrm{L}$ goodness-of-fit test. A high $\mathrm{H}$-L statistic is related to a low $P$ value and indicates lack of $\mathrm{fit}^{17}$.

Discrimination is the model's ability to distinguish women who will - from those who will not develop early-onset recurrence ${ }^{18}$. We quantified discrimination by calculating the area under the receiver operating characteristic (ROC) curve ${ }^{19}$. This can range from $50 \%$ (the flip of a coin) to $100 \%$ (perfect discrimination). The difference in the areas under the ROC curves of the cohort that was used for developing the model and this external validation cohort was tested using the method of DeLong et $a l^{20}$, extended for both paired and unpaired ROC curves. This is a non-parametric method to compare two empirical ROC curves.

\section{Performance of the model for the prediction of other outcomes}

We performed two disease spectrum validations to test how transportable the model is to different domains of patients. First, we redefined recurrence as having PE requiring delivery before 37 weeks of pregnancy in one analysis, and we performed another analysis on PE irrespective of gestational duration. We computed the area under the ROC curve when using the predicted probabilities of the model to predict these two endpoints. The second disease-spectrum validation served to assess the model's ability to discriminate between women who will - and those who will not develop a maternal placental syndrome. Again, we computed the area under the ROC curve to determine the model's discriminative ability. 
Finally, to determine the clinical applicability of the prediction model to create subgroups of women based on similar risk (e.g. low-risk and high-risk groups), we constructed a table comparing sensitivity, specificity, and positive- (PPV) and negative predictive values (NPV) for different risk-thresholds.

We used SPSS version 20 (IBM Corp. Released 2011) for imputation of the data. All statistical analyses were performed using $\mathrm{R}$, a language and environment for statistical computing, version 2.15.1.

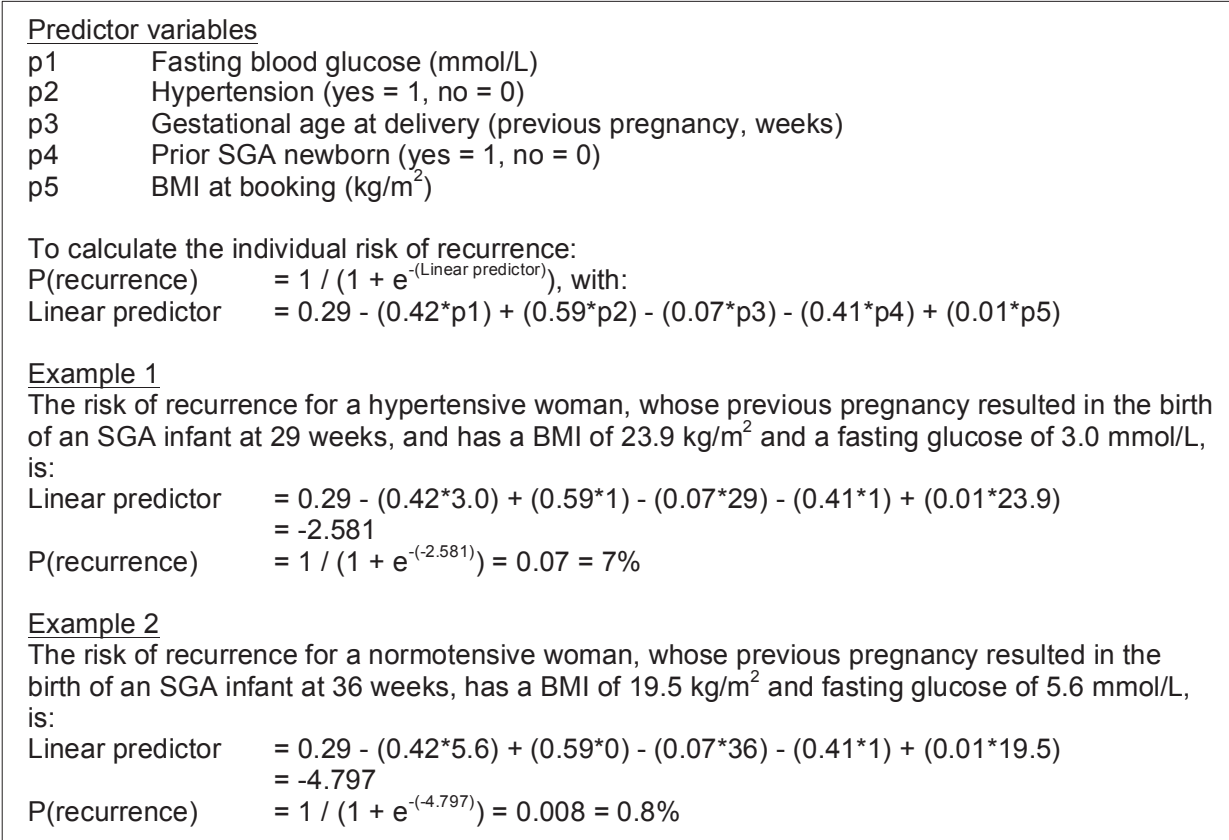

Box 1 Preconception prediction model for the risk of recurrence of early-onset preeclampsia

\section{Results}

\section{Patient population}

We included a total of 229 women, of whom 152 were collected prospectively, and 77 were retrieved from hospital patient registries. Table 1 shows patients' characteristics at booking, details about their previous pregnancy, and variables measured at interpregnancy screening. 
Table 1 Characteristics of study population ${ }^{\mathrm{a}}$

\begin{tabular}{lcc}
\hline & $\begin{array}{c}\text { Observations } \\
\text { missing }(\%)\end{array}$ & $\begin{array}{c}\text { Total cohort } \\
(\mathrm{N}=229)\end{array}$ \\
\hline Patient characteristics & & \\
Age at conception $(\mathrm{y})$ & 0.0 & $30.8(4.8)$ \\
Body mass index $\left(\mathrm{kg} / \mathrm{m}^{2}\right)$ & 19.1 & $25.5(5.4)$ \\
Hypertension, $\mathrm{n}(\%)$ & 22.6 & $58(32.6)$ \\
Parity at start pregnancy, median $=1$ & 0.0 & \\
1, $\mathrm{n}(\%)$ & & $212(92.2)$ \\
$2, \mathrm{n}(\%)$ & & $13(5.7)$ \\
$>2, \mathrm{n}(\%)$ & 19.1 & $5(2.1)$ \\
Systolic blood pressure at booking $(\mathrm{mmHg})$ & $122.4(13.9)$ \\
Diastolic blood pressure at booking $(\mathrm{mmHg})$ & $75.9(11.3)$ \\
Characteristics of previous pregnancy & & \\
Gestational age at delivery (days) & 0.0 & $207(20)$ \\
Infant's birthweight (gr) & 30.4 & $1072(453)$ \\
SGA newborn (<10th centile), $\mathrm{n}(\%)$ & 10.9 & $84(41.0)$ \\
Eclampsia, $\mathrm{n}(\%)$ & 7.0 & $11(5.1)$ \\
IUFD, $\mathrm{n}(\%)$ & 5.7 & $35(16.1)$ \\
Postpartum screening & & \\
Fasting glucose (mmol/L) & 24.8 & $5.0(0.7)$ \\
\hline
\end{tabular}

${ }^{\mathrm{a}}$ Data are given as mean \pm SD or as absolute value (\%)

Table 2 lists the outcome of the target pregnancy for both the entire cohort, and the subgroups that did or did not develop recurrent early-onset PE. The rate of early-onset recurrence of $\mathrm{PE}$ was $6.6 \%$, which is almost identical to the recurrence rate in the cohort used to develop the model (6.9\%).

\section{Model performance}

The average predicted risk was $3.0 \%$ (standard deviation $(S D)=1.5 \%$ ), which is lower than the observed recurrence rate (6.6\%). This indicates an overall underestimation of recurrence risk in this cohort, as computed by our prediction model. Furthermore, the small SD of the predicted risk indicates that most predictions were relatively close to the average. The calibration plot is shown in figure 1 . The triangles represent mean recurrence risk (with error bars representing 95\% confidence intervals), for patients with similar risk. This figure illustrates that predictions on average underestimate the actual risk over the entire range of predictions. The $\mathrm{H}-\mathrm{L}$ Chi-square statistic yielded a $P$ value of 0.05 , which indicates a statistically significant deviation from good model fit. This is consistent with the observed measures of calibration. The area under the ROC curve (figure 2) was 59\% (95\% Cl: $45-73$ ), compared with 65\% (after correction for overfitting) in the development sample. The difference was not statistically significant $(P=0.19)$. An area under the ROC curve of about $59 \%$ is consistent with poor discriminative ability. 


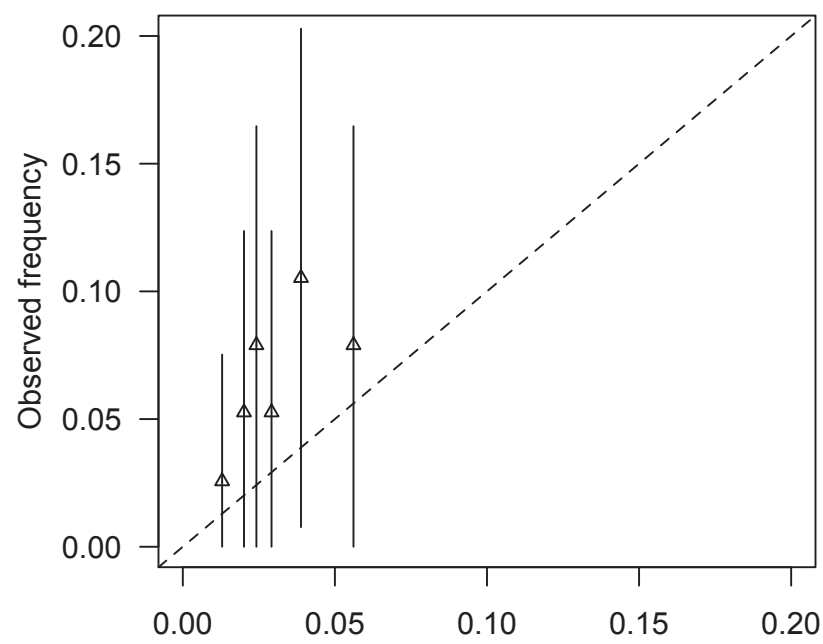

Predicted probability

Figure 1 Calibration plot of the prediction model in the external validation cohort

Table 2 Outcome of subsequent pregnancy ${ }^{a}$

\begin{tabular}{lccc}
\hline Characteristic & $\begin{array}{c}\text { Total cohort } \\
(\mathrm{N}=229)\end{array}$ & $\begin{array}{c}\text { Early-onset recurrence } \\
(\mathrm{N}=15)\end{array}$ & $\begin{array}{c}\text { No recurrence } \\
(\mathrm{N}=214)\end{array}$ \\
\hline Recurrence of preeclampsia & & & - \\
$\quad$ Recurrence $<34$ weeks, $\mathrm{n}(\%)$ & $15(6.6)$ & - & - \\
Recurrence $<37$ weeks, $\mathrm{n}(\%)$ & $25(11.0)$ & - & - \\
All-onset recurrence, $\mathrm{n}(\%)$ & $43(19.8)$ & - & $38.0(3.0)$ \\
Characteristics of subsequent pregnancy & & & $2962(813)$ \\
Gestational age at delivery (weeks) & $37.4(3.6)$ & $30.0(2.6)$ & $49(22.9)$ \\
Birthweight (gr) & $2839(920)$ & $1291(535)$ & $0(0)$ \\
SGA newborn (<10th centile), $\mathrm{n}(\%)$ & $53(23.0)$ & $4(26.7)$ & $5(2.3)$ \\
Eclampsia, $\mathrm{n}(\%)$ & $1(0.5)$ & $1(6.7)$ & $1(6.7)$ \\
IUFD, $\mathrm{n}(\%)$ & $6(2.6)$ & & \\
\hline
\end{tabular}

${ }^{\mathrm{a}}$ Data are given as mean \pm SD or as percentages

\section{Recalibration}

To correct for systematic differences between observed recurrence rate and average predicted risk, the model's intercept can be readjusted. This modifies the average predicted risk, but the spread in predictions and the relative ranking remain unaltered and thus this modification does not affect the discriminative ability of the model. We computed that a perfect calibration-in-the-large for this cohort was obtained when the intercept was set at 1.11 instead of 0.29 in the derivation cohort. 
For future use, a weighted mean intercept of 0.59 based on the number of patients in both the derivation and external validation cohorts is recommended.

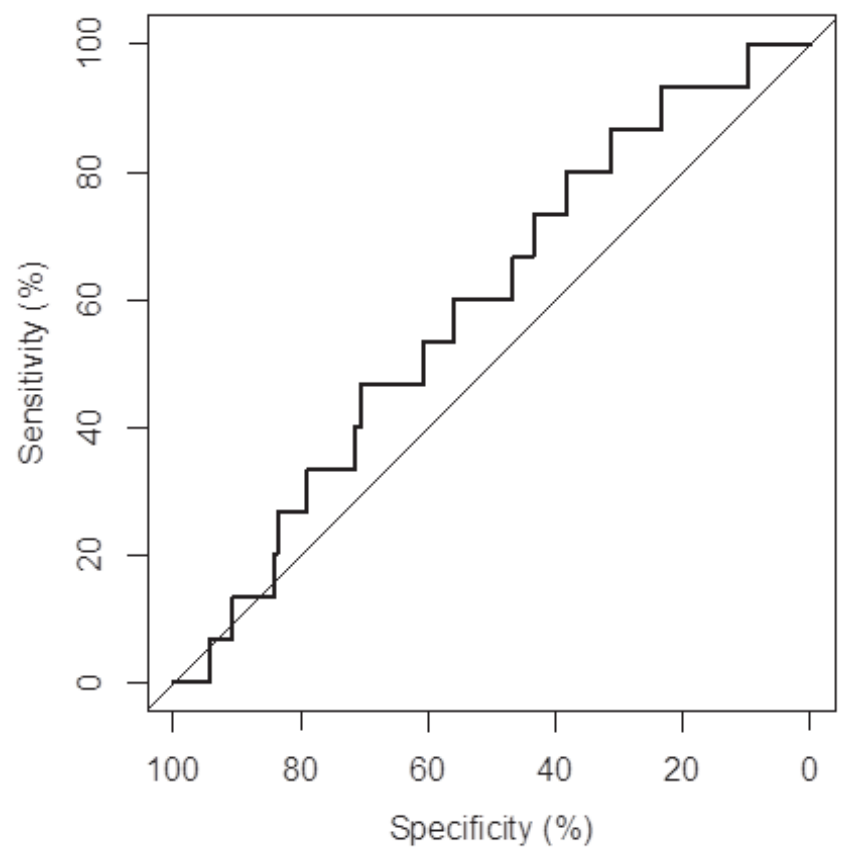

Figure $\mathbf{2}$ ROC curve of the prediction model in the external validation cohort

Table 3 Different predicted risk thresholds and the corresponding sensitivities, specificities and predictive values

\begin{tabular}{|c|c|c|c|c|c|}
\hline & $\begin{array}{l}\text { Risk threshold }^{a} \\
(\%)\end{array}$ & $\begin{array}{c}\text { Sensitivity }^{\mathrm{b}} \\
(\%)\end{array}$ & $\begin{array}{c}\text { Specificity }^{\mathrm{b}} \\
(\%)\end{array}$ & $\begin{array}{c}\mathrm{PPV}^{\mathrm{b}} \\
(\%)\end{array}$ & $\begin{array}{l}\mathrm{NPV}^{\mathrm{b}} \\
(\%)\end{array}$ \\
\hline \multirow[t]{4}{*}{$\overline{\mathrm{PE}}$} & 2.0 & $93.3(14 / 15)$ & $10.3(22 / 214)$ & $6.8(14 / 206)$ & $95.7(22 / 23)$ \\
\hline & 3.0 & $80.0(12 / 15)$ & $32.7(70 / 214)$ & $7.7(12 / 156)$ & $95.9(70 / 73)$ \\
\hline & 3.5 & $60.0(9 / 15)$ & $52.3(112 / 214)$ & $8.1(9 / 111)$ & $94.9(112 / 118)$ \\
\hline & 4.0 & $46.7(7 / 15)$ & $62.6(134 / 214)$ & $8.0(7 / 87)$ & $94.4(134 / 142)$ \\
\hline
\end{tabular}

Abbreviations: PPV, positive predictive value; NPV, negative predictive value.

${ }^{a}$ Women were considered test-positive if their predicted risk was at or above this level.

${ }^{\mathrm{b}}$ Data in parenthesis represent proportions.

\section{Clinical applicability}

Table 4 lists prognostic accuracy measures for 4 different risk thresholds to classify women into a low-risk and a high-risk subgroup. We computed recurrence risks after calculating the new intercept. The table shows that, when using a risk threshold of e.g. $3.5 \%$ (i.e. women with a predicted risk at or above this level are considered high risk), 
more than half of all women (118/229) were considered at low risk for recurrence of early-onset PE. However, PE still recurs in $4 \%$ of this low-risk group ( $100 \%-N P V)$, which is only slightly less frequent than the $7.7 \%$ observed recurrence rate (=PPV) in the high-risk group as well as the à priori probability of $6.6 \%$ in the entire cohort. These posterior probabilities (i.e. probabilities of recurrence within risk categories) are consistent with poor discriminating power of the model to differentiate between former patients who will and will not develop recurrent PE. This conclusion holds for all risk thresholds listed in table 4.

Performance of the model for the prediction of other outcomes

Adopting later-onset PE, PE irrespective of onset, or placental syndrome as outcome variables increased the number of events and precision of the performance estimates (table 3). The model discriminates slightly better when used to predict later-onset $(<$ 37 weeks) as well as recurrence of all PE. Interestingly, the model performed considerably better (AUC: 66\%, 95\% Cl: 55 - 77) when used to discriminate between women who would, and would not develop maternal placental syndrome.

Table 4 Results of the disease spectrum validation

\begin{tabular}{lccc}
\hline & $\begin{array}{c}\text { Preeclampsia } \\
<37 \text { weeks }\end{array}$ & All preeclampsia & $\begin{array}{c}\text { Placental syndrome } \\
<34 \text { weeks }\end{array}$ \\
\hline Recurrence rate $(\%)$ & 11.0 & 19.8 & 9.1 \\
Mean predicted risk $(\%)$ & $\mathrm{NA}^{\mathrm{a}}$ & $\mathrm{NA}^{\mathrm{a}}$ & $\mathrm{NA}^{\mathrm{a}}$ \\
AUC $(95 \% \mathrm{Cl})$ & $62.4 \%(51.0-73.7)$ & $61.4 \%(51.9-70.9)$ & $65.8 \%(54.7-76.9)$ \\
\hline
\end{tabular}

${ }^{\mathrm{a}}$ The initial model was developed to estimate the risk of recurrence before 34 weeks and therefore, unsuitable to estimate the risk $<37$ weeks, preeclampsia irrespective of gestational age or placental syndrome. Therefore, no mean predicted risk can be calculated. Nevertheless, determining discriminative ability, which is based on relative ranks of predicted probabilities, is possible.

\section{Discussion}

This study aimed to externally validate a previously developed prediction model intended to be used at intake for antenatal care in apparently healthy women, who experienced early-onset PE in their previous pregnancy. Among these former patients, a suitable test would enable identifying the ones at low risk of developing recurrent disease. Ideally - in case of a sensitive test - low-risk women could be offered similar prenatal care as low-risk primigravidas, thus not only avoiding superfluous medicalization and diagnostic testing, but also reducing healthcare costs.

In this study we externally validated a pre-pregnancy prediction model for the risk of recurrence of early-onset PE in a cohort of 229 former patients from secondary and tertiary care centres. To our knowledge, our study is the first to externally validate a recurrence risk prediction model for PE. Other studies have assessed associations of individual or combined predictor variables with recurrence but no external validation was carried out ${ }^{21-26}$. 
Compared to the derivation and internal validation of the model, the performance in the external validation cohort was lower. The area under the ROC curve was only 59\%, with poor calibration. On average, the model underestimates predicted probabilities over the entire range. In addition, the range and spread of probabilities were small. Predictions by a perfectly calibrated model would range from 0 to $100 \%$, with predictions preferably covering this entire interval. In this validation cohort the predicted probabilities only ranged roughly from 0 to $10 \%$, with a SD of only $1.5 \%$. Thus, in this external validation sample, we found our model to perform poorly and thus to be unsuitable for identifying women that could be offered antenatal care similar to low-risk primigravidas.

To improve model performance, an individual patient data (IPD) meta-analysis could be performed to combine data from different cohorts together with both derivation and external validation data to study the added performance when including promising new predictor variables. Promising new predictors may be e.g. placental abruption in the previous pregnancy ${ }^{27}$, maternal thrombophilia ${ }^{28}$, high-density lipoprotein (HDL) cholesterol $^{26}$, plasma volume ${ }^{29,30}$, and homeostasis model assessment of insulin resistance (HOMA-IR) ${ }^{31}$. We did not update the model using these predictors yet, because of the relatively small number of recurrences in our cohort. Keeping in mind the rule of thumb of 10 events per variable ${ }^{32}$, over 750 women with a history of early-onset PE and an ongoing next pregnancy are needed to evaluate the added value of these 5 predictors.

Our model predicted maternal placental syndrome reasonably well, even though the model was not developed for this purpose. This may be explained by the current view that the pathophysiology of PE and other maternal placental syndromes are at least in part overlapping, in particular with respect to placental insufficiency and/or dysfunction ${ }^{12}$. Limiting outcome to recurrent PE only implies that a large proportion of patients with placental syndrome with a different phenotype than PE will be excluded. In order to study the ability to predict maternal placental syndrome and classify women into risk categories using easily obtainable pre-pregnancy predictors, a prediction model should be developed specifically for this set of adverse events. To date, no such model has been reported.

A limitation of this prediction model by design is that it only uses plausible parameters known before pregnancy. Such a model does not include parameters becoming only abnormal in the course of a pregnancy, which eventually becomes complicated by PE or other forms of the placental syndrome. The absence of these pregnancy-dependent parameters in the model implies that the model can be expected to perform worse than a model that also includes specific pregnancy-dependent parameters. Although our model based on pre-pregnancy variables performs poorly in the prediction of a recurrent $\mathrm{PE}$, its performance is likely to improve considerably when in early midpregnancy, pregnancy-specific variables, such as Flt-1 and placental growth factor, are incorporated in the model ${ }^{33}$. 
Another limitation of our study was the number of missing values for some predictor variables. Omitting patients with one or more missing predictor variables from the analysis would reduce statistical power markedly, and may introduce bias ${ }^{15,19}$. Although regression-based imputation is often preferred over most commonly used methods as, for instance, imputation using the (subgroup) mean, its use does not guarantee unbiased estimates. If data are missing according to a not at random mechanism, regression-based imputation may introduce bias ${ }^{15}$. However, in the original PreCare dataset we measured a wide range of variables that were included in the imputation model, increasing the likelihood of valid predictions of replacements for missing values.

Our study provides an empirical example of the importance of an external validation. The initial model performed well, even after correcting in an internal validation step. Unfortunately, external validation studies are relatively rare compared to model development studies, yet they yield so much information on the expected performance in populations that are similar the cohort used for external validation.

Conclusion: We externally validated a model to estimate the individual recurrence risk of early-onset PE and placental syndrome. Discriminative ability and calibration of the model were poor. The model was unable to distinguish clinically relevant subgroups of women according to their recurrence risk. 


\section{References}

1. Sibai B, Dekker G, Kupferminc M. Pre-eclampsia. Lancet. Feb 26-Mar 4 2005;365(9461):785-799.

2. Barton JR, Sibai BM. Prediction and prevention of recurrent preeclampsia. Obstet Gynecol. Aug 2008;112(2 Pt 1):359-372.

3. Hernandez-Diaz S, Toh S, Cnattingius S. Risk of pre-eclampsia in first and subsequent pregnancies: prospective cohort study. Bmj. 2009;338:b2255.

4. Langenveld J, Jansen S, van der Post J, Wolf H, Mol BW, Ganzevoort W. Recurrence risk of a delivery before 34 weeks of pregnancy due to an early onset hypertensive disorder: a systematic review. American journal of perinatology. Aug 2010;27(7):565-571.

5. van Kuijk SM, Nijdam ME, Janssen KJ, et al. A model for preconceptional prediction of recurrent early-onset preeclampsia: derivation and internal validation. Reprod Sci. Nov 2011;18(11):1154-1159.

6. Steyerberg EW. Clinical Prediction Models. New York: Springer; 2009.

7. Royston P, Moons KG, Altman DG, Vergouwe Y. Prognosis and prognostic research: Developing a prognostic model. Bmj. 2009;338:b604.

8. Toll DB, Janssen KJ, Vergouwe Y, Moons KG. Validation, updating and impact of clinical prediction rules: a review. J Clin Epidemiol. Nov 2008;61(11):1085-1094.

9. Delahaije DH, van Kuijk SM, Dirksen CD, et al. Cost-effectiveness of recurrence risk guided care versus care as usual in women who suffered from early-onset preeclampsia including HELLP syndrome in their previous pregnancy (the PreCare study). BMC Pregnancy Childbirth. 2010;10:60.

10. Brown MA, Lindheimer MD, de Swiet M, Van Assche A, Moutquin JM. The classification and diagnosis of the hypertensive disorders of pregnancy: statement from the International Society for the Study of Hypertension in Pregnancy (ISSHP). Hypertens Pregnancy. 2001;20(1):IX-XIV.

11. Sibai BM. Diagnosis, controversies, and management of the syndrome of hemolysis, elevated liver enzymes, and low platelet count. Obstet Gynecol. May 2004;103(5 Pt 1):981-991.

12. Granger JP, Alexander BT, Llinas MT, Bennett WA, Khalil RA. Pathophysiology of hypertension during preeclampsia linking placental ischemia with endothelial dysfunction. Hypertension. Sep 2001;38(3 Pt 2):718-722.

13. Ray JG, Vermeulen MJ, Schull MJ, Redelmeier DA. Cardiovascular health after maternal placental syndromes (CHAMPS): population-based retrospective cohort study. Lancet. Nov 19 2005;366(9499):1797-1803.

14. The Netherlands Perinatal Registery [homepage on the internet]. Bilthoven: NPRfoundation; [cited 2008 November 21]. Available from: http://www.perinatreg.nl/.

15. Allison PD. Missing Data. lowa: Sage; 2001.

16. Steyerberg EW, Vickers AJ, Cook NR, et al. Assessing the performance of prediction models: a framework for traditional and novel measures. Epidemiology. Jan 2010;21(1):128-138.

17. Hosmer DW, Lemeshow S. Applied Logistic Regression. New York: John Wiley \& Sons, Inc.; 2000.

18. Hanley JA, McNeil BJ. The meaning and use of the area under a receiver operating characteristic (ROC) curve. Radiology. Apr 1982;143(1):29-36.

19. Frank E. Harrell J. Regression Modeling Strategies. New York: Springer; 2001.

20. Delong E, DeLong D, Clarke-Pearson D. Comparing the Areas under Two or More Correlated Receiver Operating Characteristic Curves: A Nonparametric Approach. Biometrics. 09// 1988;44(3):837-845. 
21. Langenveld J, Buttinger A, van der Post J, Wolf H, Mol BW, Ganzevoort W. Recurrence risk and prediction of a delivery under 34 weeks of gestation after a history of a severe hypertensive disorder. BJOG. Apr 2011;118(5):589-595.

22. Rasmussen S, Irgens LM, Albrechtsen S, Dalaker K. Predicting preeclampsia in the second pregnancy from low birth weight in the first pregnancy. Obstet Gynecol. Nov 2000;96(5 Pt 1):696-700.

23. Bramham K, Briley AL, Seed P, Poston L, Shennan AH, Chappell LC. Adverse maternal and perinatal outcomes in women with previous preeclampsia: a prospective study. $A m \mathrm{~J}$ Obstet Gynecol. Jun 2011;204(6):512 e511-519.

24. McDonald SD, Best C, Lam K. The recurrence risk of severe de novo pre-eclampsia in singleton pregnancies: a population-based cohort. BJOG. Nov 2009;116(12):1578-1584.

25. Melamed N, Hadar E, Peled Y, Hod M, Wiznitzer A, Yogev Y. Risk for recurrence of preeclampsia and outcome of subsequent pregnancy in women with preeclampsia in their first pregnancy. J Matern Fetal Neonatal Med. Nov 2012;25(11):2248-2251.

26. Sep SJ, Smits LJ, Prins MH, Spaanderman ME, Peeters LL. Simple prepregnant prediction rule for recurrent early-onset hypertensive disease in pregnancy. Reprod Sci. Jan 2009;16(1):80-87.

27. Melamed N, Hadar E, Peled Y, Hod M, Wiznitzer A, Yogev Y. Risk for recurrence of preeclampsia and outcome of subsequent pregnancy in women with preeclampsia in their first pregnancy. 20121015 2012(1476-4954 (Electronic)).

28. Facchinetti F, Marozio L, Frusca T, et al. Maternal thrombophilia and the risk of recurrence of preeclampsia. Am J Obstet Gynecol. Jan 2009;200(1):46 e41-45.

29. Aardenburg R, Spaanderman ME, van Eijndhoven HW, de Leeuw PW, Peeters LL. A low plasma volume in formerly preeclamptic women predisposes to the recurrence of hypertensive complications in the next pregnancy. I Soc Gynecol Investig. Dec 2006;13(8):598-603.

30. Scholten RR, Sep S, Peeters L, Hopman MT, Lotgering FK, Spaanderman ME. Prepregnancy low-plasma volume and predisposition to preeclampsia and fetal growth restriction. Obstet Gynecol. May 2011;117(5):1085-1093.

31. Hauth JC, Clifton RG, Roberts JM, et al. Maternal insulin resistance and preeclampsia. Am J Obstet Gynecol. Apr 2011;204(4):327 e321-326.

32. Peduzzi P, Concato J, Kemper E, Holford TR, Feinstein AR. A simulation study of the number of events per variable in logistic regression analysis. J Clin Epidemiol. Dec 1996;49(12):1373-1379.

33. Kuc S, Wortelboer EJ, van Rijn BB, Franx A, Visser GH, Schielen PC. Evaluation of 7 serum biomarkers and uterine artery Doppler ultrasound for first-trimester prediction of preeclampsia: a systematic review. Obstet Gynecol Surv. Apr 2011;66(4):225-239. 


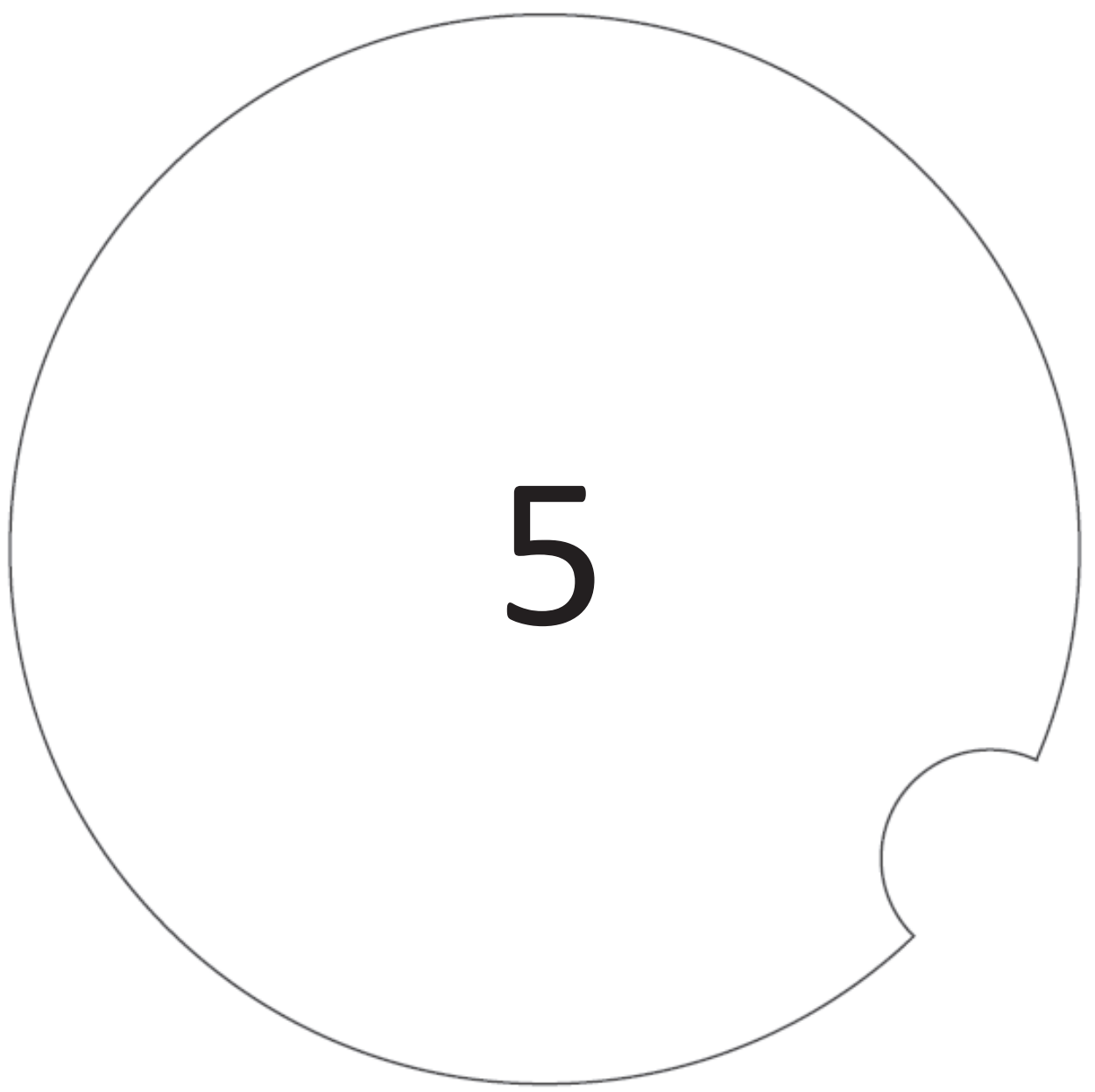

\section{Multicentre impact analysis of a model for predicting recurrent early-onset preeclampsia}

van Kuijk SM, Delahaije DH, Dirksen CD, Scheepers HC, Spaanderman ME, Ganzevoort W, Duvekot JJ, Oudijk MA, van Pampus MG, Peeters LL, and Smits LJ Submitted 


\begin{abstract}

\section{Objective}

To determine the impact of customized antenatal care in the next pregnancy in women with a history of early-onset preeclampsia (PE) after early-pregnancy allocation to medium care $(\mathrm{MC})$ and high care $(\mathrm{HC})$ subgroups on the basis of a model predicting their recurrence risk.
\end{abstract}

\title{
Methods
}

We compared hospital care consumption of risk-based subgroups and compared pregnancy outcome and patient's self-reported health state between this recurrencerisk-guided care (RGC) type with a reference group receiving "standard" care as dictated by local protocol (care-as-usual, CAU).

\section{Results}

We included a total of 311 women from 12 hospitals. In the RGC group ( $N=159$ ), women in the $\mathrm{MC}$ group received less outpatient visits and diagnostic testing compared to women in the $\mathrm{HC}$ group. Compared to CAU $(\mathrm{N}=152)$, implementing the model for evidence-based care stratification did not lead to different pregnancy outcomes or self-perceived health scores. On average, care consumption of the MC and $\mathrm{HC}$ groups combined did not differ from CAU.

\section{Conclusion}

Our study exemplifies that recurrence risk-based stratification of antenatal care in former preeclampsia patients is feasible, can affect patient management, does not lead to worse maternal and foetal outcomes. Stratifying clinical management according to expected risk may not only help to reassure former patients during their next pregnancy, it may also lower the individual and societal burden of potentially excessive surveillance. 


\section{Introduction}

In the Netherlands, most women with a history of early-onset preeclampsia (PE), with or without the syndrome of haemolysis, elevated liver enzymes and low platelets (HELLP) are counselled before their next pregnancy. During this counselling visit the majority is screened for subclinical disorders known to predispose to recurrence of hypertensive pregnancy disorders. Even though evidence for a lower PE recurrence rate after intensified antenatal care is lacking, the clinical management of a next pregnancy often consists of far more antenatal visits and serial antenatal (biophysical and biochemical) surveillance than clinically indicated. Several large studies report that early-onset PE only recurs in approximately 4 to $6 \%^{1-3}$ of pregnant women. Thus, identifying women at low recurrence risk among former patients may not only help to reassure these women, but also motivate the care provider to be more restrictive in offering extensive antenatal care to these women, when their pregnancy is evolving normally.

Previously, we developed a model for pre-pregnancy prediction of recurrent earlyonset $\mathrm{PE}^{4}$. Analysis of risk cut-off values showed that the model was promising for the identification of a large proportion of women who are at low risk. However, before widespread implementation of a prediction model as a decision aid, it is recommended to perform an impact analysis ${ }^{5-8}$. In this particular case, it serves to verify whether implementing a prediction model in routine clinical practice has an impact on patient management, without negative effects on clinical outcomes. We hypothesize that implementation of our model does neither result in a higher rate of adverse pregnancy outcome compared to standard care, nor does it lead to more depression, more anxiety or a lower quality of life of these former patients receiving less extensive, customized care. In fact, less emphasis on possible negative outcome may even result in better psychological outcome. Results of an impact study for our prediction model could help to decide whether more individualized risk-based care is justified. This would contribute not only to a more efficient and responsible use of the available resources, but also "protect" low-risk patients from the burden of excessive surveillance.

The objective of this study was to evaluate the impact of recurrence-risk guided care (RGC) in the next pregnancy in women with a history of early-onset preeclampsia (PE) after early-pregnancy allocation to low-risk and high-risk subgroups on the basis of a model predicting their individual recurrence risk. Such an impact analysis consists of 1 ) determining whether caregivers apply the model and use its result, and 2) determining whether implementation of the model has an impact on patients, compared to a scenario in which no such model is used. To do so, we first compared care consumption between the Medium Care and High Care subgroups, which were differentially managed according to risk-based antenatal care protocols. We then compared pregnancy outcomes and psychological well-being in the RGC group to women who received care-as-usual (CAU), i.e. women who received antenatal care according to local protocol, without stratification based on predicted recurrence risk. 


\section{Methods}

This study is part of the PreCare project (pregnant women with previous preeclampsia: efficiency of care guided by recurrence-risk). In this project, the cost-effectiveness of recurrence-risk guided care (RGC) compared to care as usual (CAU) is evaluated. The design of the PreCare project has been reported previously in greater detail ${ }^{9}$.

\section{Design of the impact study}

In a before-after design, we made a comparison between women enrolled after implementation of the prediction model (i.e. RGC) to those enrolled before (i.e. CAU). In the CAU phase of the study, women were treated according to local protocol. After the CAU part, we implemented the prediction model as a decision aid for gynaecologists in an easy-to-use web-based application, and made it available to all participating centres. The predictor variables were required to be checked in tick boxes (dichotomous variables) or filled in a text box (continuous variables). The fields used for continuous variables were secured in a way that only values from a range of likely values could be filled in. For women who delivered before 34 weeks in their previous pregnancy, the prediction model was used to determine whether they required extensive antenatal surveillance (i.e. High Care, or $\mathrm{HC}$ ) or less extensive antenatal care (i.e. Medium Care, or $\mathrm{MC}$ ) would suffice. After filling in all predictor variables, the resulting output of the model was either $\mathrm{MC}$ for women below or at a predefined cutoff point or HC for women with a model result above that particular cut-off level. Women who gave birth between 34 and 37 weeks pregnancy were all considered at low risk for recurrence before 34 weeks, and therefore offered MC. A schematic representation of the design can be found in figure 1. Assessment of recurrence risk and communication of the related antenatal care protocol was performed - if feasible at the first visit, but always before 17 weeks pregnancy. Since pre-conception communication of the recurrence risk may influence the choice whether or not to proceed with a next pregnancy, and the model was not yet externally validated, the model was not allowed to be used for pre-pregnancy counselling. In addition, such selection based on recurrence risk may cause selection bias ${ }^{10}$. 


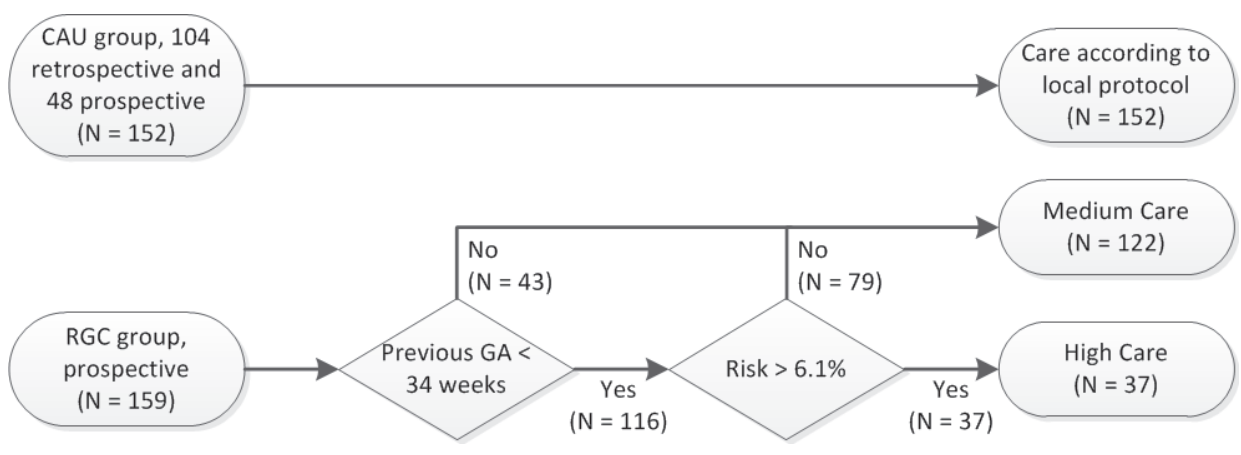

Figure 1 Flowchart of antenatal care strategy of both the care as usual (CAU) group and the recurrence-risk guided care $(\mathrm{RGC})$ group

\section{Patients}

We prospectively enrolled pregnant women from 6 academic hospitals and 6 tertiary referral centres in the Netherlands between August 2008 and May 2012. Local research nurses, midwives, and gynaecologists, who screened the appointment system on a daily basis, assessed the eligibility for inclusion of these women at the outpatient clinic. Women eligible for inclusion were pregnant with an amenorrhea of less than 17 weeks, and had experienced preeclampsia and/or the HELLP syndrome in their previous pregnancy resulting in delivery for medical reasons before 37 weeks pregnancy. We excluded women from participation who were younger than 18 or who had one of the following comorbidities: diabetes mellitus, systemic lupus erythematosus, antiphospholipid syndrome, heart disease or kidney disease.

Using patient registries from these hospitals we retrospectively included women who already had a recorded subsequent ongoing pregnancy. Women from this retrospective cohort were matched to women from the prospective cohort for age (2025 years, 25-30 years, 30-35 years, 35-40 years, 40 years and above), duration of previous pregnancy (<34 weeks or $34-37$ weeks), and hospital region. These women were added to the prospective CAU cohort to yield a balanced design.

\section{Prediction model and risk stratification}

We developed the prediction model using 5 preselected predictor variables (fasting glucose level, body mass index, gestational age at birth of the previous complicated pregnancy, the birth of a small-for-gestational-age (SGA) infant, and presence or absence of chronic hypertension). A newborn was considered SGA when its birth weight was below the $10^{\text {th }}$ centile for gestational age at delivery, based on the most recent Dutch population charts $^{11}$. The formula used to calculate the individual recurrence risk of early-onset PE is shown in box 1 . We defined a cut-off value of $6.1 \%$ (i.e. women below or at this level are considered test-negative, women above that level are considered positive) to stratify women into MC (all test-negative women) and $\mathrm{HC}$ (all test-positive women) groups using the prediction model. This was determined using the empirical data of the derivation cohort. We stipulated that the risk threshold 
needed to secure an expected rate of recurrence of early-onset PE in the MC group to be below $5 \%$, while also enabling an as large as possible proportion of women to be allocated to the MC group. The latter precondition for the model was expected to result in the best possible individualized prenatal care in these women. Utilizing a risk cut-off of $6.1 \%$ resulted in an average predicted risk in the test-negative group of $<5 \%$, yet keeping the proportion selected as large as possible.

\section{Monitoring protocols}

A number of gynaecologists of the participating centres were approached to participate in a consensus process, during which the final antenatal care protocols were designed. They were all obstetricians specialized in hypertensive disorders of pregnancy. After reaching consensus, the $\mathrm{MC}$ and $\mathrm{HC}$ protocols and information on how to use the online prediction model were distributed among all obstetric care providers participating in the study, by means of on-site presentations by one of the researchers (SVK) or a local research nurse. Furthermore, we distributed pocket-cards containing all relevant study information, including the protocols. Both antenatal care protocols are described in Chapter 2.

\section{Primary and secondary outcomes}

The primary endpoint of this study was the impact of using our prediction model in the RGC group for stratification of patient management. This was reflected in the difference between the $\mathrm{MC}$ and $\mathrm{HC}$ groups, in number of outpatient antenatal visits, ultrasound exams, automated blood pressure recordings, and blood and urine samplings for laboratory testing. The secondary endpoint was the difference between the CAU setting and the RGC setting with respect to pregnancy outcomes and maternal and foetal morbidity and mortality. To this end, we collected data on the occurrence of early-onset PE and/or HELLP, and the occurrence of any PE and/or HELLP irrespective of gestational age at delivery. In addition, we evaluated differences between RGC and $\mathrm{CAU}$ with respect to the incidence of eclampsia, pregnancy duration, infant's birth weight, incidence of SGA births, and psychological well-being. All endpoints were recorded during the first ongoing pregnancy after a previous pregnancy complicated with PE or HELLP. 


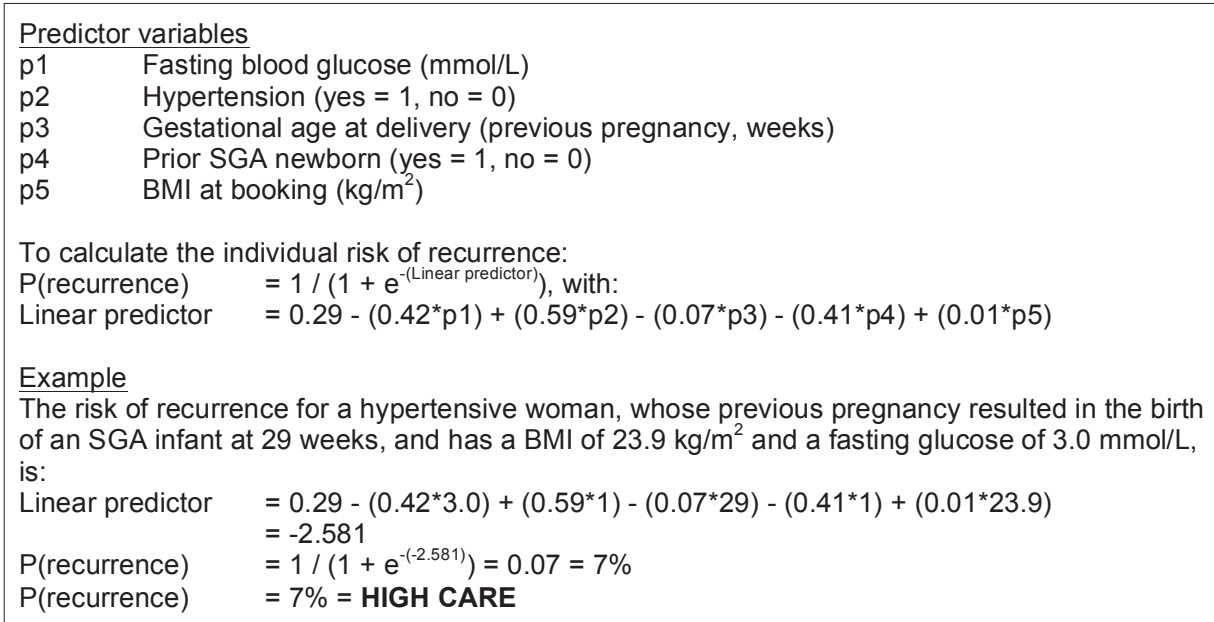

Box 1 Preconception prediction model for the risk of recurrence of early-onset preeclampsia

\section{Psychological measurements}

To compare CAU to RGC with respect to psychological well-being, we used 4 different questionnaires at 3 time points. We measured health-related quality of life using the EuroQol 5D-3L (EQ-5D-3L) self-report questionnaire ${ }^{12}$. It is a 16-item questionnaire divided into 5 clusters: mobility, self-care, usual activities, pain/ discomfort and anxiety/ depression. These clusters can be scored on 3 levels: no problems, small problems, or serious problems. In addition, the EQ-5D-3L contains a visual analogue scale (VAS) to record ones self-perceived health, in which the end-points are labelled 'Best imaginable health state' and 'Worst imaginable health state'. We converted the EQ-5D-3L scores to a single summary index using the Dutch tariff ${ }^{13}$. The tariff is a valuation set for the scores of the EQ-5D-3L, specific for the population it was applied to $^{14}$. The resulting summary index is a utility score that ranges from -0.33 to 1 . The maximum score of 1 corresponds to no problems and highest perceived health. Posttraumatic stress disorder was estimated by the post-traumatic stress disorder (PTSD) symptom scale $(\mathrm{PSS})^{15}$. It is a 17 -items questionnaire that assesses the presence and severity of DSM-IV symptoms related to a traumatic event, in this case experiencing early-onset preeclampsia in the previous pregnancy. The total score ranges from 0 to 51. A high score corresponds to more or more severe PTSD symptoms. We measured anxiety using the State-Trait Anxiety Inventory (STAI) ${ }^{16}$. The STAI consists of 20 questions on anxiety at the present time and 20 questions about anxiety in general. We confined ourselves to current anxiety. All questions can be answered using a 4point Likert scale. The total score ranges from 20 to 80 points, higher scores being consistent with greater anxiety. We measured depression using the Beck Depression Inventory $(\mathrm{BDI})^{17}$. This 21 -questions multiple-choice self-report questionnaire measures depression. The total score ranges from $0-63$, higher scores corresponding with more depressive symptoms. 


\section{Statistical analysis}

The characteristics of the cohort at baseline were summarized using the original data before imputation of missing values. We tested for differences between the CAU and RGC groups at baseline using Student's t-test for continuous variables, and the Chisquared test for categorical variables. Fisher's exact test was used for any categorical variables that had expected cell counts of less than 5 . We considered a p-value of 0.05 or less to indicate statistical significance.

There were some variables of interest that were incomplete, i.e. some values were missing. We used regression imputation to substitute missing values. We estimated the impact of the prediction model as a decision aid using the post-imputation dataset. For the analysis of psychological outcomes, we only used data from women who completed at least one questionnaire. After this selection, the imputed data for these women was used for the analysis.

To evaluate the impact of the prediction model, we analysed differences in pregnancy outcome, number of outpatient visits, and ultrasound and laboratory testing between the $\mathrm{MC}$ and the $\mathrm{HC}$ group, both of the RGC part of the study. Depending on the scale of the variable of interest, we used either analysis of covariance (ANCOVA) or logistic regression analysis to estimate the influence of care protocol on care consumption, corrected for complications during the pregnancy (i.e. recurrence of PE or HELLP, pregnancy duration and intra-uterine foetal demise (IUFD). Next, we assessed and compared pregnancy outcomes and differences in patient management for both the CAU and RGC parts of the study.

We also determined the impact on psychological outcome of prediction-model-guided care relative to standard care by evaluating the differences between the CAU group and the 2 RGC subgroups for the baseline questionnaires (i.e. EQ-5D-3L, BDI, PSS and STAI), the questionnaires at 29 weeks and the postpartum questionnaires, filled in 10 days after delivery. For the baseline and 29 weeks-pregnant measurements, the difference was corrected for potential confounders (BMI, gravidity, chronic hypertension, age at conception, previous GA, and previous birth of an SGA baby) measured at baseline using ANCOVA. All these variables combined with pregnancy outcomes were used to adjust differences in psychological outcomes 10 days after delivery.

We used SPSS version 20 (IBM Corp. Released 2011) for imputation of the data and for all statistical analyses.

\section{Results}

We included a total number of 311 eligible women, of whom 207 were enrolled in the prospective part of the study, and 104 were collected retrospectively (figure 1). Of all women from the prospective cohort, 48 received standard care, and, together with the retrospective cohort, constitute the CAU group $(n=152)$. Baseline characteristics of 
the CAU group and RGC group ( $n=159$ ) did not differ appreciably, except for a lower percentage caesarean deliveries and SGA newborns in the previous pregnancy in the latter group (table 1).

Table 1 Baseline patient characteristics before imputation of the data ${ }^{a}$

\begin{tabular}{lccc}
\hline \multicolumn{1}{c}{ Characteristic } & $\begin{array}{c}\text { Care as usual } \\
(\mathrm{n}=152)\end{array}$ & $\begin{array}{c}\text { Recurrence-risk } \\
\text { guided care } \\
(\mathrm{n}=159)\end{array}$ & $\begin{array}{c}\text { P-value } \\
\text { for } \\
\text { difference }\end{array}$ \\
\hline Patient characteristics & & & \\
Age at conception (y) & $31.2(5.0)$ & $30.9(4.5)$ & 0.631 \\
BMI (kg/m2) & $26.3(5.6)$ & $25.6(6.0)$ & 0.324 \\
Hypertension, $\mathrm{n}(\%)$ & $27(34.2 \%)$ & $49(32.5 \%)$ & 0.791 \\
Parity, median =1 & & 0.439 \\
1, $\mathrm{n}(\%)$ & $137(90.1 \%)$ & $146(91.8 \%)$ & \\
$2, \mathrm{n}(\%)$ & $11(7.2 \%)$ & $9(5.7 \%)$ & \\
$>2, \mathrm{n}(\%)$ & $4(2.7 \%)$ & $4(2.5 \%)$ & \\
Systolic blood pressure at booking (mmHg) & $123.0(15.1)$ & $123.5(14.2)$ & 0.811 \\
Diastolic blood pressure at booking (mmHg) & $77.1(13.1)$ & $75.2(10.1)$ & 0.224 \\
Characteristics of previous pregnancy & & & \\
Gestational age at delivery (weeks) & $31.1(3.6)$ & $31.1(3.8)$ & 0.987 \\
Infant's birth weight (gr) & $1432(734)$ & $1409(730)$ & 0.822 \\
SGA newborn (<10th centile), $\mathrm{n}(\%)$ & $56(47.9 \%)$ & $48(30.4 \%)$ & 0.003 \\
HELLP-syndrome, $\mathrm{n}$ (\%) & $84(61.3 \%)$ & $82(53.9 \%)$ & 0.206 \\
Eclampsia, $\mathrm{n}$ (\%) & $9(6.8 \%)$ & $7(4.6 \%)$ & 0.237 \\
IUFD, $\mathrm{n}(\%)$ & $18(13.6 \%)$ & $19(12.3 \%)$ & 0.728 \\
Caesarean section, $\mathrm{n}(\%)$ & $121(83.4 \%)$ & $108(69.2 \%)$ & 0.004 \\
\hline
\end{tabular}

${ }^{a}$ Data are presented as mean (SD) or percentage

${ }^{\mathrm{b}} \mathrm{A}$ P-value of 0.05 or lower was considered statistically significant

Table 2 shows that pregnancy outcomes in the HC group compared to the MC group were slightly worse, although none of the differences was statistically significant. On average, pregnancy duration was shorter, newborns had lower birth weight and there were more early-onset recurrences. These differences are expected since the groups are stratified according to predicted recurrence risk. Table 3 lists the actual extensiveness of prenatal care in both subgroups, indirectly reflecting the model's effect on patient management. Women in the HC group had 2-3 extra outpatient visits $(+16 \%)$, and underwent 1-2 times more often blood and urine sampling for diagnostic testing $(+40 \%)$. These differences were statistically significant, even after adjusting for recurrence, pregnancy duration and IUFD. Although the mean duration of antepartum hospital admission appeared longer in $\mathrm{MC}$ than in $\mathrm{HC}$ (8.0 days versus 3.7 days), the difference did not reach statistical significance, most likely in conjunction with the wide variation in hospital stay after admission. 
Table 2 Subsequent pregnancy outcomes in the recurrence-risk-guided care group, stratified by care-protocol ${ }^{\mathrm{a}}$

\begin{tabular}{lccc}
\hline & $\begin{array}{c}\text { Medium Care } \\
(\mathrm{n}=122)\end{array}$ & $\begin{array}{c}\text { High Care } \\
(\mathrm{n}=37)\end{array}$ & $\begin{array}{c}\text { P-value } \\
\text { for difference }^{\mathrm{b}}\end{array}$ \\
\hline Gestational age at delivery (weeks) & $37.8(2.7)$ & $37.0(3.7)$ & 0.122 \\
Infants birth weight (gr) & $2938(690)$ & $2774(1026)$ & 0.264 \\
SGA newborn (<10th centile), $\mathrm{n}(\%)$ & $25(20.5 \%)$ & $8(21.6 \%)$ & 1.000 \\
Early-onset recurrence, $\mathrm{n}(\%)$ & $6(4.9 \%)$ & $3(8.1 \%)$ & 0.686 \\
All recurrence, $\mathrm{n}(\%)$ & $27(22.3 \%)$ & $8(21.6 \%)$ & 1.000 \\
IUFD, $\mathrm{n}(\%)$ & $0(0 \%)$ & $1(2.7 \%)$ & 0.233 \\
\hline
\end{tabular}

${ }^{\mathrm{a}}$ Data are presented as mean (SD) or frequency (percentage)

${ }^{\mathrm{b}} \mathrm{A}$ P-value of 0.05 or lower was considered statistically significant

Table 3 Care consumption in the recurrence-risk-guided care group, stratified by care protocol ${ }^{\mathrm{a}}$

\begin{tabular}{lcccc}
\hline & $\begin{array}{c}\text { Medium Care } \\
(\mathrm{n}=122)\end{array}$ & $\begin{array}{c}\text { High Care } \\
(\mathrm{n}=37)\end{array}$ & $\begin{array}{c}\text { P-value for } \\
\text { difference }^{\mathrm{b}}\end{array}$ & $\begin{array}{c}\text { P-value for } \\
\text { difference, after } \\
\text { adjustement }^{\mathrm{c}}\end{array}$ \\
\hline Outpatient visits & $14.9(5.6)$ & $17.3(5.3)$ & 0.021 & 0.006 \\
Admitted to hospital antepartum & $31(25.4 \%)$ & $11(29.7 \%)$ & 0.602 & 0.616 \\
Admission antepartum (days) & $8.0(8.1)$ & $3.7(2.2)$ & 0.096 & 0.162 \\
Admission for delivery (days) & $5.7(5.1)$ & $5.1(3.8)$ & 0.506 & 0.256 \\
Blood pressure measurements & $15.0(10.2)$ & $16.4(9.4)$ & 0.476 & 0.318 \\
Ultrasound measurement & $8.7(4.4)$ & $9.3(3.7)$ & 0.503 & 0.436 \\
Blood collected & $4.1(3.2)$ & $5.4(2.6)$ & 0.034 & 0.027 \\
Urine collected & $2.6(3.0)$ & $3.8(3.9)$ & 0.048 & 0.039
\end{tabular}

${ }^{\mathrm{a}}$ Data are presented as mean (SD) or frequency (percentage)

${ }^{b}$ A P-value of 0.05 or lower was considered statistically significant

${ }^{\mathrm{C}}$ Adjusted for recurrence of PE or HELLP, pregnancy duration and IUFD, a P-value of 0.05 or lower was considered statistically significant

Table 4 shows subsequent pregnancy outcomes in the CAU and RGC groups. We did not observe any clinically relevant differences in pregnancy outcome, except for a higher total number of intra-uterine foetal deaths (IUFD) in CAU (5 versus 1 in the RGC group). Because of the low numbers foetal deaths, we did not perform any statistical testing. In the whole cohort, the recurrence rate of early-onset PE was 6.1\% (19/311), and recurrence irrespective of gestational age at delivery was $19.6 \%(61 / 311)$. The only statistically significant difference we observed in the indicators of care consumption during pregnancy was a higher number of ultrasound exams in the RGC group compared to the CAU group (8.9 versus 7.3 , P-value $=0.001$ ). 
Table 4 Outcome of subsequent pregnancy and indicators of care consumption for the care-asusual group and the recurrence-risk-guided care group ${ }^{a}$

\begin{tabular}{lccc}
\hline & $\begin{array}{c}\text { Recurrence- } \\
\text { risk guided } \\
\text { care } \\
(\mathrm{n}=159)\end{array}$ & $\begin{array}{c}\text { P-value for } \\
\text { difference }\end{array}$ \\
\hline Characteristics of subsequent pregnancy & & & \\
$\quad$ Gestational age at delivery (weeks) & $37.4(3.9)$ & $37.6(2.9)$ & 0.291 \\
Infants birth weight (gr) & $2873(986)$ & $2900(781)$ & 0.780 \\
SGA newborn (<10th centile), $\mathrm{n}(\%)$ & $37(24.3 \%)$ & $33(20.8)$ & 0.917 \\
Early-onset recurrence, $\mathrm{n}(\%)$ & $10(6.6 \%)$ & $9(5.7 \%)$ & 0.681 \\
All recurrence, $\mathrm{n}(\%)$ & $26(18.7 \%)$ & $35(22.2 \%)$ & 0.554 \\
Eclampsia, $\mathrm{n}(\%)$ & $2(1.4 \%)$ & $0(0.0 \%)$ & $\mathrm{c}$ \\
IUFD, $\mathrm{n}$ (\%) & $5(3.3 \%)$ & $1(0.6 \%)$ & $\mathrm{c}$ \\
Caesarean section, $\mathrm{n}(\%)$ & $69(45.4 \%)$ & $65(40.9 \%)$ & 0.491 \\
Highest systolic blood pressure (mmHg) & $143.2(19,3)$ & $146.4(22.0)$ & 0.147 \\
Highest diastolic blood pressure (mmHg) & $93.3(14.3)$ & $91.8(13.5)$ & 0.485 \\
Indicators of care consumption, during pregnancy & & & \\
Outpatient visits & $16.8(7.1)$ & $15.5(5.6)$ & 0.146 \\
Admitted to hospital antepartum, $\mathrm{n}(\%)$ & $37(24.3 \%)$ & $42(26.4 \%)$ & 0.698 \\
Duration of admission antepartum (days) & $4.9(5.7)$ & $6.9(7.2)$ & 0.181 \\
Ultrasound measurements & $7.3(5.0)$ & $8.9(4.3)$ & 0.001 \\
Blood collected & $4.6(3.9)$ & $4.4(3.2)$ & 0.650 \\
Urine collected & $2.9(4.0)$ & $2.9(3.3)$ & 0.933 \\
\hline
\end{tabular}

${ }^{\mathrm{a}}$ Data are presented as mean (SD) or frequency (percentage)

${ }^{\mathrm{b}} \mathrm{A}$ P-value of 0.05 or lower was considered statistically significant

${ }^{\mathrm{C}}$ No statistical testing was performed because of the low incidence of eclampsia and IUFD

Differences between RGC and CAU with respect to depression, anxiety, post-traumatic stress, and quality of life are summarized in table 5 . The test results for these psychological features did not differ appreciably between the two groups. On average, women from the CAU group and the RGC group reported similar BDI scores indicative of a mild depression, had mildly elevated signs of anxiety, scored low on the PTSD symptom scale, and a good health state as measured by the EUROQOL-5D-3L. 
Table 5 Self-report questionnaires for both the CAU and RGC group ${ }^{a}$

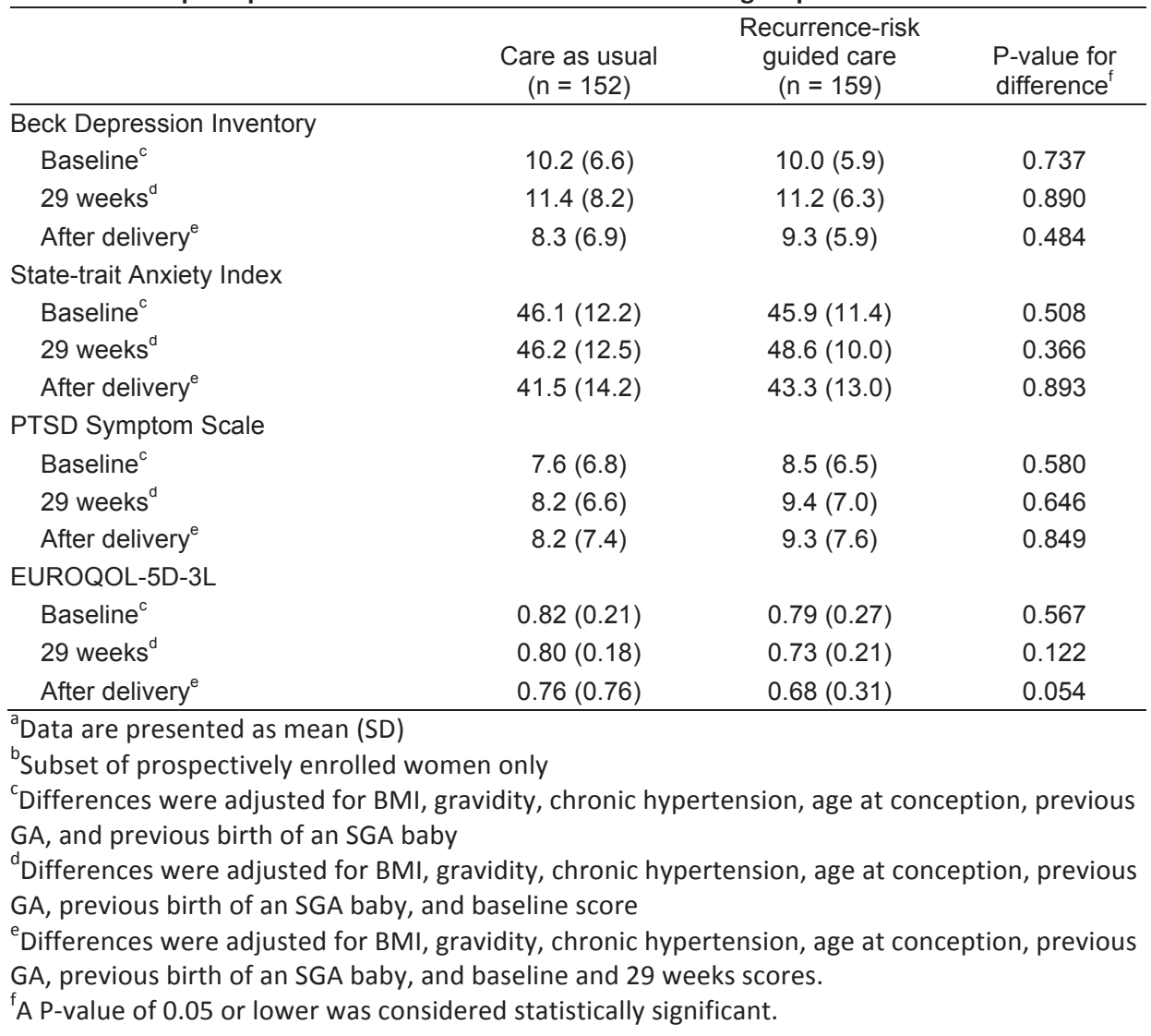

\section{Discussion}

Many prediction models have been developed for application in clinical practice, often as a decision aid. As such, a model is implemented in routine clinical practice and one or more risk cut-off points may help in clinical decision-making. Although many have emphasized the need for both an external validation and an impact analysis ${ }^{6-8,18-20}$ only few models have adhered to this prerequisite for a clinically applicable prediction tool. Only after an impact analysis may a model become suitable for use in clinical practice. We have developed a prediction model for the recurrence of early-onset preeclampsia, prospectively validated the model in patients from a broader range of hospitals, and subjected the model to a formal impact analysis.

A number of studies report on subsequent pregnancy outcomes after a first (earlyonset) preeclampsia ${ }^{1,2,21-23}$. Our study is the first to evaluate if alleviating the normally very intense antenatal care has any effect on pregnancy outcomes. In addition, we collected much information on psychological well-being both during the first ongoing pregnancy after one complicated by early-onset PE or HELLP, and after delivery. 
Our results show that implementing the prediction model has impact on patient management. On average, women who received a $\mathrm{HC}$ management had more outpatient visits and were more often subjected to blood and urine testing, and had shorter average pregnancy duration. Because of the limited number of antenatal hospital admissions with highly variable length, we were unable to test the intergroup difference in the average duration of hospital stay. Particularly the women in the HC group, tended to have much shorter hospital stays (yet more frequent). Introducing the prediction model as a decision aid to differentiate prenatal care did not have a negative impact on pregnancy outcome compared to standard care. This observation is in line with the general view that recurrent PE or HELLP cannot be prevented, nor can its clinical onset be delayed or its severity after onset be attenuated by increasing the extensiveness of antenatal care. In addition, women in the CAU and RGC groups did not differ with respect to symptoms of depression, post-traumatic stress, anxiety and quality of life. Our results indicate that implementing such risk stratification results in less extensive antenatal care, and is not harmful for the group receiving less intensified antenatal care (i.e. the MC Group).

Only months after the start of the RGC part of our study, results of the HYPITAT study were published ${ }^{24}$. In this study, which was performed in many of the same hospitals as our study, women allocated to induction of labour because of gestational hypertension or mild preeclampsia between 36 and 41 weeks, were reported to have a decreased risk of poor maternal outcome compared to similar women in the expectant management group. A subsequent study showed a considerable increase in induction of labour in women with hypertensive disease in the Netherlands, and a decrease in eclampsia prevalence compared to before the trial ${ }^{25}$. In our data, we observed fewer events of eclampsia and IUFD, although we did not statistically test this difference because of the low numbers. This may be due to the national change that may have had an influence on the management (i.e. more hospital admittances for induction of labour and subsequently shorter gestational age at delivery) of a subgroup of our patients. Because of differences in case definition of mild PE we could not determine the influence of the change in management of this subgroup on the overall results, but it is highly unlikely to alter our conclusions.

With the data from part of the participants in this impact analysis, we validated the prediction model externally (Chapter 4). We concluded that the model did not identify women at low risk for recurrence of early-onset preeclampsia with sufficient certainty. In addition, we found that the model discriminated poorly between low- and high-risk women. Even though the impact study shows an effect on patient management and no increase in hypertensive complications and foetal morbidity when using risk-based antenatal care, results of the validation study imply that the specific prediction model we used does not perform well enough to be used for risk classification of former patients.

A prospective randomized trial is the preferred design for an impact analysis. In our case, this would mean that CAU or RGC would be randomized either per patient or per hospital. Despite this preference, we chose to perform a before - after study. 
Randomizing at patient level could have been influenced markedly by cross-over: gynaecologists would be able to apply the same knowledge about the risk of recurrence of patients in the CAU group as in the RGC group, and possibly unwittingly use this knowledge as a guide for extensiveness of antenatal care, potentially diminishing possible differences. We did not choose to randomize on hospital level. The influence of local protocol on care in the hospitals assigned to the CAU group, or the adherence to the protocols offered to the hospitals assigned to the RGC group could have distorted our results. Therefore, we chose to perform a before-after study in which at least all hospitals that participated in the before part, would also participate in the part after implementation. In this study design, hospitals are both in the implementation group, as well as the control group. This is a statistically efficient method, and circumvents the problem of bias introduced by large differences between hospitals.

A limitation of our study was that some values on important variables were missing. Therefore, we used regression imputation to substitute missing values with plausible values. Even though this method is not flawless, simulation studies have shown that in most scenarios it gives unbiased results, and outperforms other methods of handling missing data. Considering only patients for the analyses that have no missing values on any of these variables could result in a dramatic decrease of sample size, and consequently in loss of statistical power ${ }^{26-29}$. The probability of a type-ll error could be inflated. In addition to this, results can be severely biased when the subset of patients who have no values missing is not a random part of the whole cohort. Finally, excluding prospectively included patients from the analysis may even be unethical.

\section{Conclusion}

The present impact study showed that patient management changed after implementing a prediction model for the recurrence risk of preeclampsia or the HELLP syndrome. In addition, it showed that offering less antenatal care visits to a majority of women with low recurrence risk did not lead to different pregnancy outcomes, or selfreported psychological well-being. 


\section{References}

1. Hernandez-Diaz S, Toh S, Cnattingius S. Risk of pre-eclampsia in first and subsequent pregnancies: prospective cohort study. BMJ. 2009;338:b2255.

2. van Rijn BB, Hoeks LB, Bots ML, Franx A, Bruinse HW. Outcomes of subsequent pregnancy after first pregnancy with early-onset preeclampsia. Am J Obstet Gynecol. Sep 2006;195(3):723-728.

3. Langenveld J, Jansen S, van der Post J, Wolf H, Mol BW, Ganzevoort W. Recurrence risk of a delivery before 34 weeks of pregnancy due to an early onset hypertensive disorder: a systematic review. American journal of perinatology. Aug 2010;27(7):565-571.

4. van Kuijk SM, Nijdam ME, Janssen KJ, et al. A model for preconceptional prediction of recurrent early-onset preeclampsia: derivation and internal validation. Reprod Sci. Nov 2011;18(11):1154-1159.

5. McGinn TG, Guyatt GH, Wyer PC, Naylor CD, Stiell IG, Richardson WS. Users' guides to the medical literature: XXII: how to use articles about clinical decision rules. Evidence-Based Medicine Working Group. JAMA. Jul 5 2000;284(1):79-84.

6. Moons KG, Altman DG, Vergouwe $Y$, Royston P. Prognosis and prognostic research: application and impact of prognostic models in clinical practice. BMJ. 2009;338:b606.

7. Toll DB, Janssen KJ, Vergouwe $Y$, Moons KG. Validation, updating and impact of clinical prediction rules: a review. J Clin Epidemiol. Nov 2008;61(11):1085-1094.

8. Reilly BM, Evans AT. Translating clinical research into clinical practice: impact of using prediction rules to make decisions. Annals of internal medicine. Feb 7 2006;144(3):201209.

9. Delahaije DH, van Kuijk SM, Dirksen CD, et al. Cost-effectiveness of recurrence risk guided care versus care as usual in women who suffered from early-onset preeclampsia including HELLP syndrome in their previous pregnancy (the PreCare study). BMC Pregnancy Childbirth. 2010;10:60.

10. Van Kuijk SM, Sep SJ, Nelemans PJ, Smits LJ. How long do preconception risk prediction models hold? Influence of selective fertility on model performance. Paediatric and perinatal epidemiology. Nov 2010;24(6):602-607.

11. The Netherlands Perinatal Registery [homepage on the internet]. Bilthoven: NPRfoundation; [cited 2008 November 21]. Available from: http://www.perinatreg.nl/.

12. EuroQol--a new facility for the measurement of health-related quality of life. The EuroQol Group. Health Policy. Dec 1990;16(3):199-208.

13. Lamers LM, Stalmeier PF, McDonnell J, Krabbe PF, van Busschbach JJ. [Measuring the quality of life in economic evaluations: the Dutch EQ-5D tariff]. Nederlands tijdschrift voor geneeskunde. Jul 9 2005;149(28):1574-1578.

14. Dolan P, Roberts J. Modelling valuations for Eq-5d health states: an alternative model using differences in valuations. Med Care. May 2002;40(5):442-446.

15. Foa EB, Riggs DS, Dancu CV, Rothbaum BO. Reliability and validity of a brief instrument for assessing post-traumatic stress disorder.

16. Spielberger C, Gorsuch R, Lushene R, Vagg P, Jacobs G. Manual for the State-Trait Anxiety Inventory. Palo Alto, CA: Consulting Psychologists Press; 1983.

17. Beck AT, Ward $\mathrm{CH}$, Mendelson M, Mock J, Erbaugh J. An inventory for measuring depression. Arch Gen Psychiatry. Jun 1961;4:561-571.

18. Laupacis A, Sekar N, Stiell IG. Clinical prediction rules. A review and suggested modifications of methodological standards. JAMA. Feb 12 1997;277(6):488-494.

19. Royston P, Moons KG, Altman DG, Vergouwe Y. Prognosis and prognostic research: Developing a prognostic model. BMJ. 2009;338:b604.

20. Steyerberg EW. Clinical Prediction Models. New York: Springer; 2009. 
21. Bramham K, Briley AL, Seed P, Poston L, Shennan AH, Chappell LC. Adverse maternal and perinatal outcomes in women with previous preeclampsia: a prospective study. Am J Obstet Gynecol. Jun 2011;204(6):512 e511-519.

22. McDonald SD, Best C, Lam K. The recurrence risk of severe de novo pre-eclampsia in singleton pregnancies: a population-based cohort. BJOG. Nov 2009;116(12):1578-1584.

23. Melamed N, Hadar E, Peled Y, Hod M, Wiznitzer A, Yogev Y. Risk for recurrence of preeclampsia and outcome of subsequent pregnancy in women with preeclampsia in their first pregnancy. J Matern Fetal Neonatal Med. Nov 2012;25(11):2248-2251.

24. Koopmans $\mathrm{CM}$, Bijlenga $\mathrm{D}$, Groen $\mathrm{H}$, et al. Induction of labour versus expectant monitoring for gestational hypertension or mild pre-eclampsia after 36 weeks' gestation (HYPITAT): a multicentre, open-label randomised controlled trial. Lancet. Sep 19 2009;374(9694):979988.

25. van der Tuuk K, Koopmans CM, Groen H, Mol BW, van Pampus MG. Impact of the HYPITAT trial on doctors' behaviour and prevalence of eclampsia in the Netherlands. Bjog. Dec 2011;118(13):1658-1660.

26. Allison PD. Missing Data. lowa: Sage; 2001.

27. Donders AR, van der Heijden GJ, Stijnen T, Moons KG. Review: a gentle introduction to imputation of missing values. J Clin Epidemiol. Oct 2006;59(10):1087-1091.

28. Janssen KJ, Donders AR, Harrell FE, Jr., et al. Missing covariate data in medical research: to impute is better than to ignore. J Clin Epidemiol. Jul 2010;63(7):721-727.

29. van der Heijden GJ, Donders AR, Stijnen T, Moons KG. Imputation of missing values is superior to complete case analysis and the missing-indicator method in multivariable diagnostic research: a clinical example. J Clin Epidemiol. Oct 2006;59(10):1102-1109. 


\section{Part 2: Methodology and statistics regarding prediction studies}

"What if you haven't the data?"

"Then we shall proceed directly to the brandy and cigars."

Lindsay Faye 


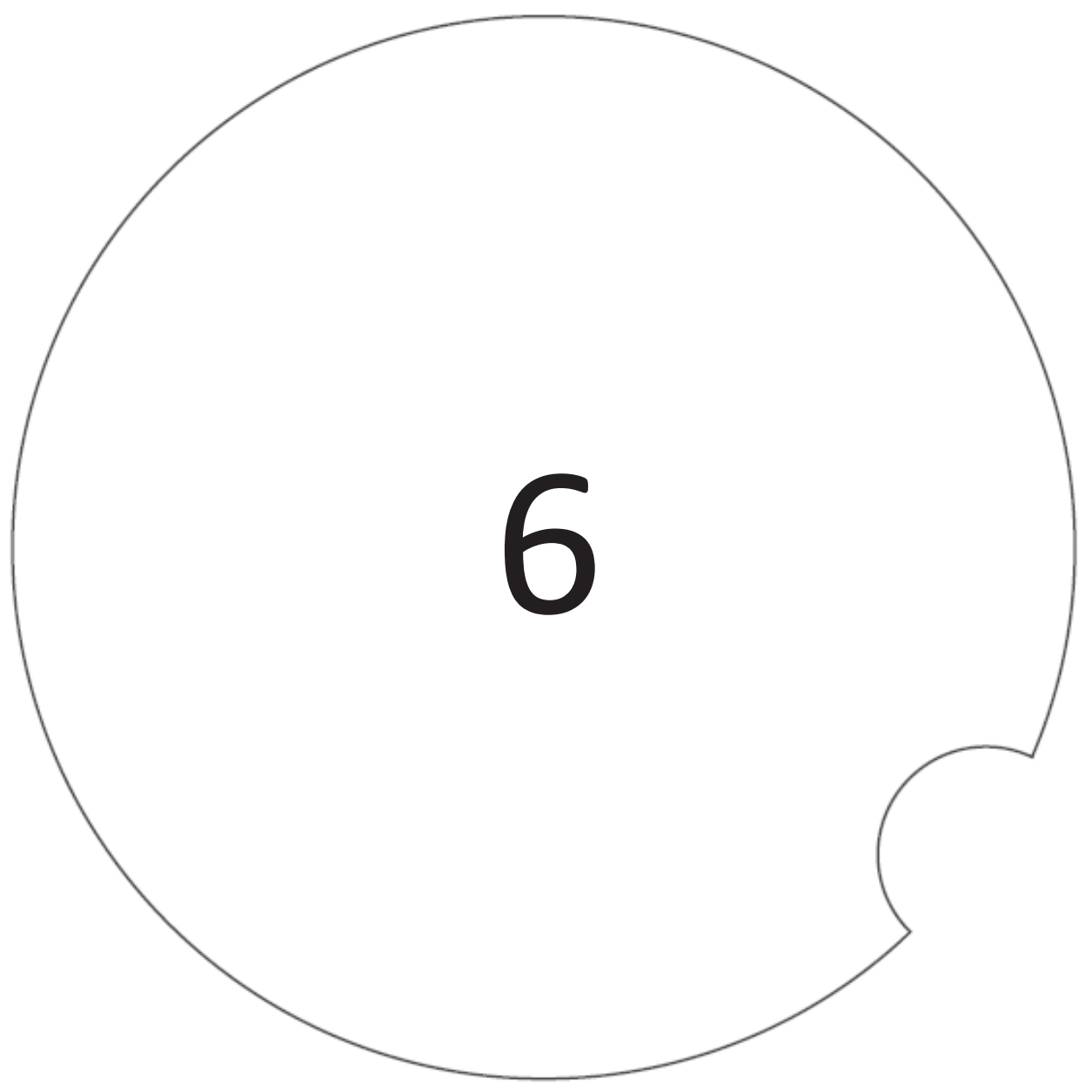

How long do preconception risk prediction models hold? Influence of selective fertility on model performance

van Kuijk SM, Sep SJ, Nelemans PJ, Smits LJ Paediatric and Perinatal Epidemiology 2010; 24: 602-607 


\begin{abstract}
Risk estimates derived from preconception prediction models can be used to counsel women with regard to any future pregnancies. Women with a high predicted risk of an adverse pregnancy outcome may decide more often not to try for another pregnancy again than women with a low predicted risk. This prediction guided selective fertility can cause a change in the composition of the pregnant population with respect to those parameters that are comprised in the prediction model. The question can be raised whether such a change in composition could influence performance parameters of the prediction model, such as sensitivity, specificity, positive- and negative predictive values and discriminative ability, when evaluating risks in the new population and whether it could compromise the longevity of the model.
\end{abstract}

Using a hypothetical example, we show that the original sensitivity and specificity estimates of a preconception prediction model for an adverse pregnancy outcome do not hold anymore when the model is applied in a population affected by model-based selective fertility: sensitivity decreases, while specificity increases. However, individual patient risk estimates remain unbiased and discriminative ability, expressed as the area under the receiver operating characteristic (ROC) curve, remains unaffected. In diagnostic research, several types of bias with a common underlying mechanism have been described. 


\section{Introduction}

Multivariable models for the prediction of individual risks of adverse health outcomes are increasingly being developed and used within a wide range of clinical disciplines. In gynaecology, models have been developed for the prediction of, among others, fertility after in vitro insemination ${ }^{1}$, small-for-gestational-age fetuses ${ }^{2}$, preeclampsia $^{3}$, and recurrence of preeclampsia ${ }^{4}$, Some models estimate risks of adverse reproductive outcome on the basis of preconception factors only, while others (additionally) take parameters into account that are measured during the target pregnancy.

Individual risk estimates derived from preconception prediction models may be used for counselling purposes and the optimization of preconception and antepartum care. In this way, the result of preconception prediction models may influence women's choices whether or not to try for a subsequent pregnancy. Such influence could be regarded as a variant of selective fertility, a phenomenon earlier defined as the tendency to adjust reproduction after the outcome of previous pregnancies ${ }^{5}$. Women with a high predicted risk of an adverse pregnancy outcome may decide more often not to get pregnant again than women with a low predicted risk, and vice versa. This prediction-guided type of selective fertility may thus cause changes in the composition of the population of pregnant women with respect to those parameters that are comprised in the prediction model. The question can be raised whether such a change in composition can have implications for the future predictive ability of the model.

In this paper, using a hypothetical example of a prediction model for the recurrence of preeclampsia with simulated data, we will show that predictive sensitivity and specificity at different cut-off points of predicted probabilities can change under the influence of selective fertility, while the discriminative ability of the model as well as individual risk predictions remain unaffected.

\section{Methods}

\section{Measures of model performance}

Predicted probabilities, derived from the prediction model, that exceed a pre-specified cut-off point are usually defined as a positive test result, while values below the prespecified cut-off point are considered a negative test result. Frequently used measures for prediction model performance are predictive sensitivity and specificity. Sensitivity is the number of subjects with a positive test result who develop the outcome, divided by the total number of subjects who develop the outcome. Specificity, on the other hand, is the number of subjects with a negative test who do not develop the outcome, divided by the total number of subjects who do not develop the outcome. Sensitivity and specificity are inversely related, and if both parameters are computed for several cut-off values, a receiver operating characteristic (ROC) curve can be plotted. The area under the ROC curve (AUC), or the concordance statistic (c-statistic), is a measure of the discriminative ability of the model. The AUC represents the chance that a randomly selected subject who develops the outcome has a higher test score than a randomly selected subject who doesn't develop the outcome. 
Two performance measures that are often more relevant to clinical practice are the positive and negative predictive value of a test. The positive predictive value is the number of subjects with a positive test result who develop the outcome, divided by the total number of subjects with a positive test result. The negative predictive value is the proportion of subjects with a negative test who do not develop the outcome, divided by the total number of subjects with a negative test result. The positive and negative predictive values thus denote the chance of developing the disease (or its complement), given the outcome of the test.

\section{Illustration}

Consider the hypothetical data of 5000 women who experienced severe preeclampsia in their first pregnancy. We emphasize that all following assumptions are made for simulation purposes. We assumed that, although all women in the population wished to get pregnant again, 20 percent of them refrained from trying because of fear of recurrent disease. Not having been exposed to the future prediction rule, these women represented a random selection of the base population.

In order to keep the illustration straightforward, we further assumed that all women who tried to get pregnant, succeeded in achieving an ongoing pregnancy, so that consecutive pregnancy outcomes are known for all of the remaining 4000 women. This population will henceforth be referred to as the derivation population (table 1).

Table 1 Base population of women with preeclampsia in their first pregnancy ( $N=5000)$, the subpopulation of women who try for another pregnancy (derivation population, $\mathrm{N}=4000$ ), and the numbers of recurrent preeclampsia in the derivation population

\begin{tabular}{cccc}
\hline $\begin{array}{c}\text { No. of risk } \\
\text { factors } \\
\text { present }\end{array}$ & Base population & Derivation population & Recurrent preeclampsia \\
\hline 4 & $\mathrm{~N}$ & $\mathrm{~N}(\%$ of base population) & $\mathrm{N}(\%$ of derivation population) \\
3 & 500 & $400(80 \%)$ & $100(25 \%)$ \\
2 & 750 & $600(80 \%)$ & $120(20 \%)$ \\
1 & 1000 & $800(80 \%)$ & $120(15 \%)$ \\
0 & 1250 & $1000(80 \%)$ & $100(10 \%)$ \\
Total & 1500 & $1200(80 \%)$ & $60(5 \%)$ \\
\hline
\end{tabular}

Suppose a simple prediction model was developed using recorded data of the derivation population, in which individual risk estimates of recurrent preeclampsia were determined by the presence or absence of one or more of a total of 4 risk factors. Extremely simplified, the mean risk of recurrence among women with no risk factors present was set at 5\%, and 10,15, 20 and $25 \%$ among women with 1, 2, 3 and 4 risk factors present, respectively. In order to achieve a high sensitivity, women with a predicted risk $>5 \%$ were set to test positive. For this specific cut-off point, sensitivity, specificity, positive predictive value and negative predictive value were computed. Using estimates of sensitivity and specificity for all predefined cut-off points, the ROC curve was plotted and the area under the curve was estimated. After development, 
the validity of this prediction model was proven in an independent population, and it was decided to apply the model in routine clinical practice. To this end, the prediction model outcome was dichotomized; women with a positive test result were categorized as "high risk", and those with a negative test result as "low risk".

Next, consider the population in which the prediction model is applied (henceforth referred to as the application population). In this population of 5000 women, which is in essence the same as the base population, from which we obtained the derivation population, the choice to try to get pregnant again is not random, but it is now influenced by the predicted risk according to the prediction model (i.e. high risk or low risk). In this scenario, we stipulated that $40 \%$ of all high risk women decide to try for subsequent pregnancy (and succeed), while all low risk women are reassured and try (and succeed in) getting pregnant. Thus, only a subset of the application population chooses to get pregnant again, conditional on predicted risk of recurrent preeclampsia. Using data of this selection $(\mathrm{N}=2900)$ of the application population, performance parameters of the prediction model were again estimated, and compared to values obtained in the derivation population.

\section{Results}

Since the aim is to illustrate bias as a result of selective fertility, only point estimates are reported, without any confidence intervals. Table 1 shows the data of the 4000 women from which the prediction model was derived. Lower risks of recurrent preeclampsia are more prevalent than higher risks. Of these women, 1200 (30\%) had no risk factors, corresponding to a low risk of recurrent preeclampsia, and thus a negative test result; 2800 (70\%) women had one or more risk factors, corresponding to a high risk of recurrence and a positive test result. In total, 500 women (12.5\%) developed recurrent preeclampsia.

Table 2 shows the cross tabulation of predicted results (positive and negative test result) and the observed pregnancy outcome (i.e. absence or presence of recurrent preeclampsia), based on the risk cutoff point of $>5 \%$ for a positive test result. Of 500 women who developed recurrent preeclampsia, 440 had a positive test result, corresponding to a sensitivity of $88 \%$. Specificity was 33\%, because 1140 of 3500 women who did not develop recurrent preeclampsia, had a negative test result. Of 2800 women with a positive test result, 440 developed recurrent preeclampsia (positive predictive value was $16 \%$ ), and 1140 of 1200 women with a negative test result did not develop recurrent preeclampsia (negative predictive value was $95 \%$ ). The discriminative ability of the model in the derivation population, quantified by the area under the ROC curve, was approximately 0.67 (figure 1 , black graph). 
Table 2. Cross tabulation of predicted outcomes (positive or negative test result) and observed recurrence of preeclampsia in the derivation population $(\mathrm{N}=4000)$ and corresponding test characteristics. A positive test result refers to the presence of at least one risk factor.

\begin{tabular}{lccc}
\hline & Recurrent preeclampsia & No recurrent preeclampsia & Total \\
\hline Positive test & 440 & 2360 & 2800 \\
Negative test & 60 & 1140 & 1200 \\
Total & 500 & 3500 & 4000 \\
\hline
\end{tabular}

Sensitivity $=(440 / 500) * 100 \%=88 \%$

Specificity $=(1140 / 3500) * 100 \%=33 \%$

Positive predictive value $=(440 / 2800) * 100 \%=16 \%$

Negative predictive value $=(1140 / 1200) * 100 \%=95 \%$

Data of the women of the application population who chose to try for a subsequent pregnancy $(\mathrm{N}=2900)$ are shown in table 3 . The composition of this population, with respect to the presence of the risk factors, was considerably different than that of the derivation population, because of selective fertility. In this case 1400 (48\%) of women had 1 or more risk factors present, and 1500 (52\%) had no risk factors present. Of 2900 women, 295 (10\%) developed recurrent preeclampsia.

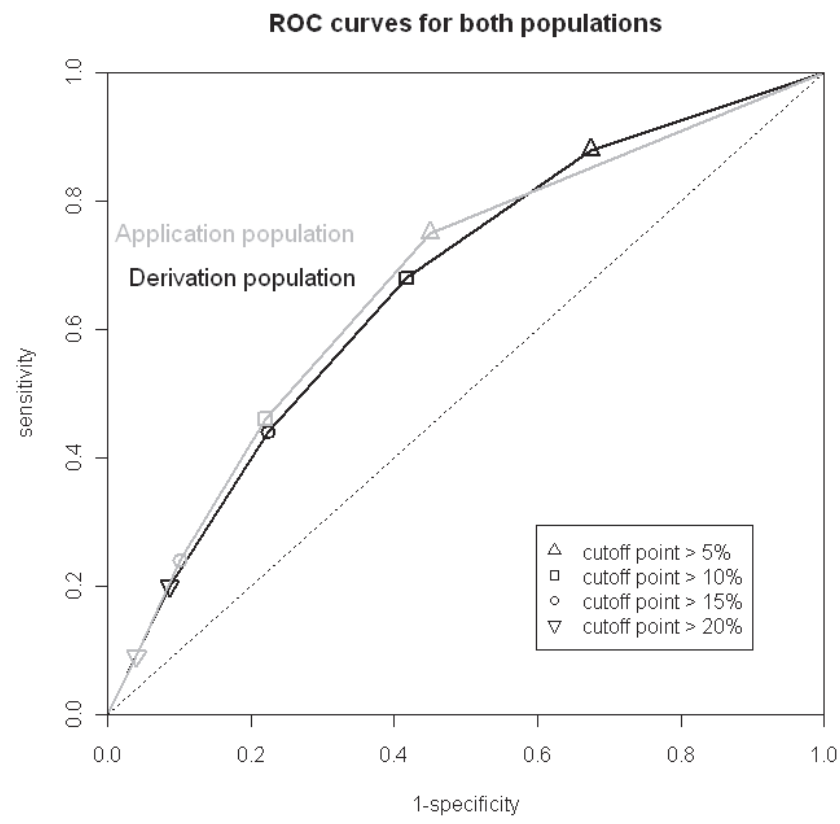

Figure 1 ROC curves of the prediction model in the derivation population and in the subset of the application population who tried for subsequent pregnancy. Points in the graph correspond to the different predefined risk-cut-off points of the prediction model result 
Table 3 Application population of women with preeclampsia in their first pregnancy ( $N=5000)$, the subpopulation who were exposed to the prediction model result and try for another pregnancy $(\mathrm{N}=2900)$, and the numbers of recurrent preeclampsia

\begin{tabular}{cccc}
\hline $\begin{array}{c}\text { No. of risk factors } \\
\text { present }\end{array}$ & $\begin{array}{c}\text { Application } \\
\text { population }\end{array}$ & $\begin{array}{c}\text { Subset who try for another } \\
\text { pregnancy }\end{array}$ & $\begin{array}{c}\text { Recurrent } \\
\text { preeclampsia } \\
\text { N (\% of subset) }\end{array}$ \\
\hline 4 & 500 & N (\% of application population) & $50(25 \%)$ \\
3 & 750 & $200(40 \%)$ & $60(20 \%)$ \\
2 & 1000 & $400(40 \%)$ & $60(15 \%)$ \\
1 & 1250 & $500(40 \%)$ & $50(10 \%)$ \\
0 & 1500 & $1500(100 \%)$ & $75(5 \%)$ \\
Total & 5000 & 2900 & 295 \\
\hline
\end{tabular}

The cross tabulation of predicted results and observed pregnancy outcomes for women from the application population who tried for a subsequent pregnancy shows the number of patients with true positive and true negative test results and false positive and false negative test results (table 4). Sensitivity of the prediction model in these data was $75 \%, 220$ of 295 women who developed recurrent preeclampsia had a positive test result. Of 2605 women who did not develop recurrent preeclampsia, 1425 had a negative test result, corresponding to a specificity of $55 \%$. Positive and negative predictive values were the same as in the derivation population, 16\% (220/1400) and $95 \%$ (1425/1500) respectively. The ROC curve of the model is depicted in figure 1 (grey graph); the area under the curve was approximately 0.67 .

Table 4 Cross tabulation of predicted outcomes (positive or negative test result) and observed recurrence of preeclampsia in the application population $(N=2900)$ and corresponding test characteristics. A positive test result refers to the presence of at least one risk factor

\begin{tabular}{lccc}
\hline & Recurrent preeclampsia & No recurrent preeclampsia & Total \\
\hline Positive test & 220 & 1180 & 1400 \\
Negative test & 75 & 1425 & 1500 \\
Total & 295 & 2605 & 2900 \\
\hline
\end{tabular}

Sensitivity $=(220 / 295) * 100 \%=75 \%$, Specificity $=(1425 / 2605) * 100 \%=55 \%$, Positive predictive value $=(220 / 1400) * 100 \%=16 \%$, Negative predictive value $=(1425 / 1500) * 100 \%=95 \%$ 


\section{Discussion}

We have illustrated how exposure of women to the results of a preconception risk prediction model could change performance parameters of the model. In our example, women with a high predicted risk of recurrent disease were less likely to try for subsequent pregnancy. We observed a decrease in sensitivity from $88 \%$ to $75 \%$ when the model was evaluated in the subset of the application population of whom next pregnancy outcomes were available, whereas specificity increased from $33 \%$ to $55 \%$. Individual risk predictions remained unaffected; in the application population both positive and negative predictive values remained the same as in the derivation population. Furthermore, the discriminative ability of the model in the subset of the application population, as quantified by the area under the ROC curve, was comparable to that of the derivation population. Even though the area under the curve remains virtually unchanged, the different cut-off points in the ROC curve migrate to the lower left corner, a phenomenon described earlier in another context ${ }^{6}$. This is because of the decrease in sensitivity (cut-off point moves down on the $y$-axis) combined with an increase in specificity (cut-off point moves left on the x-axis).

Sensitivity and specificity are of less clinical relevance than predictive values, but are still popular measures of performance in prognostic and diagnostic research, because of the false belief that they are fixed properties of a test. It is often stated that, when introducing a test in a different population, only the positive and negative predictive values are subject to change. In our simulation we have shown that because of selective fertility, and thus a selection conditional on test result, sensitivity and specificity change while, remarkably, the predictive values do not change. This might seem contrary to expectation, because predictive values depend on prevalence, which changes in our scenario $(12.5 \%$ in the derivation population versus $10 \%$ in the subset of the application population who tried for subsequent pregnancy). The explanation is quite straightforward. Because of the selection conditional on test result instead of selection conditional on disease status, in our cross tabulations (tables 2 and 4), the ratios between numbers of women with positive and negative test results (within the columns) change rather than those between numbers of women with or without recurrent preeclampsia.

Although this selective fertility-related form of bias has not been described yet in prognostic research, in diagnostic research, biases with a common underlying mechanism have been identified. These biases are referred to as work-up bias ${ }^{7}$, verification bias ${ }^{8}$, or referral bias ${ }^{6}$. Patients with a positive test result tend to be referred more often for verification by the gold standard than patients with a negative test result (selection conditional on test result). Thus, the probability of verification of disease is higher in subjects with a positive test result. As a result, sensitivity is overestimated and specificity underestimated. In the present example from prognostic research, it is the other way around. 
Our example is undoubtedly a simplified version of the truth. In the base population, we assumed that the choice to try for a subsequent pregnancy was random over the risk groups. However, this assumption may not hold. In a more realistic scenario, it is likely that the choice to try for a next pregnancy, without knowing an individual risk estimate, is amongst other factors guided by the severity of the first occurrence of preeclampsia. Severity of the first occurrence of preeclampsia is most likely related to the risk of recurrent preeclampsia (although it was not comprised in our prediction model), and therefore selection might not be random with respect to the risk groups. This may imply that in our example the strength of the bias was overestimated. On the other hand, perinatal loss is associated with severity of preeclampsia and thus associated with a risk of recurrence, yet reproduction is increased after a perinatal loss $^{9}$.

Our scenario of a preconception prediction model estimating the risk of an adverse pregnancy outcome is probably the only scenario wherein this phenomenon can occur. Patients altering their risk profile (e.g. lifestyle changes) after risk estimation or counselling can be observed in many disciplines, yet the choice to be 'at risk' or have an absolute risk of zero is unique to pregnancy-related outcomes (i.e. women who do not get pregnant again will never develop an adverse pregnancy outcome). This makes it hard to generalize this bias to other clinical disciplines.

In summary, using a simulated example, we have shown that the original sensitivity and specificity estimates of a preconception prediction model for an adverse pregnancy outcome can become biased when the model is applied in practice. However, positive and negative predictive values, as well as the discriminative ability of the model, remain unbiased. Therefore, the prediction model still yields valid individual risk estimates, and can still be used to evaluate risks and counsel these women accordingly. 


\section{References}

1. Lintsen AM, Eijkemans MJ, Hunault CC, Bouwmans CA, Hakkaart L, Habbema JD, et al. Predicting ongoing pregnancy chances after IVF and ICSI: a national prospective study. Human Reproduction. 2007; 22:2455-2462.

2. Pihl K, Larsen T, Krebs L, Christiansen M. First trimester maternal serum PAPP-A, beta-hCG and ADAM12 in prediction of small-for-gestational-age fetuses. Prenatal Diagnosis. 2008; 28:1131-1135.

3. Herraiz I, Arbues J, Camano I, Gomez-Montes E, Graneras A, Galindo A. Application of a first-trimester prediction model for pre-eclampsia based on uterine arteries and maternal history in high-risk pregnancies. Prenatal Diagnosis. 2009.

4. Sep SJ, Smits LJ, Prins MH, Spaanderman ME, Peeters LL. Simple prepregnant prediction rule for recurrent early-onset hypertensive disease in pregnancy. Reproductive Sciences. 2009; 16:80-87.

5. Skjærven R, Melve KK. Selective fertility - the examples of perinatal death and preeclampsia. Norsk Epidemiologi. 2007; 17:175 - 180.

6. Knottnerus JA, Leffers P. The influence of referral patterns on the characteristics of diagnostic tests. Journal of Clinical Epidemiol. 1992; 45:1143-1154.

7. Begg CB. Biases in the assessment of diagnostic tests. Statistics in Medicine. 1987; 6:411423.

8. Ransohoff DF, Feinstein AR. Problems of spectrum and bias in evaluating the efficacy of diagnostic tests. New England Journal of Medicine. 1978; 299:926-930.

9. Skjærven R, Wilcox AJ, Lie RT, Irgens LM. Selective fertility and the distortion of perinatal mortality. American Journal of Epidemiology. 1988; 128:1352-1363. 


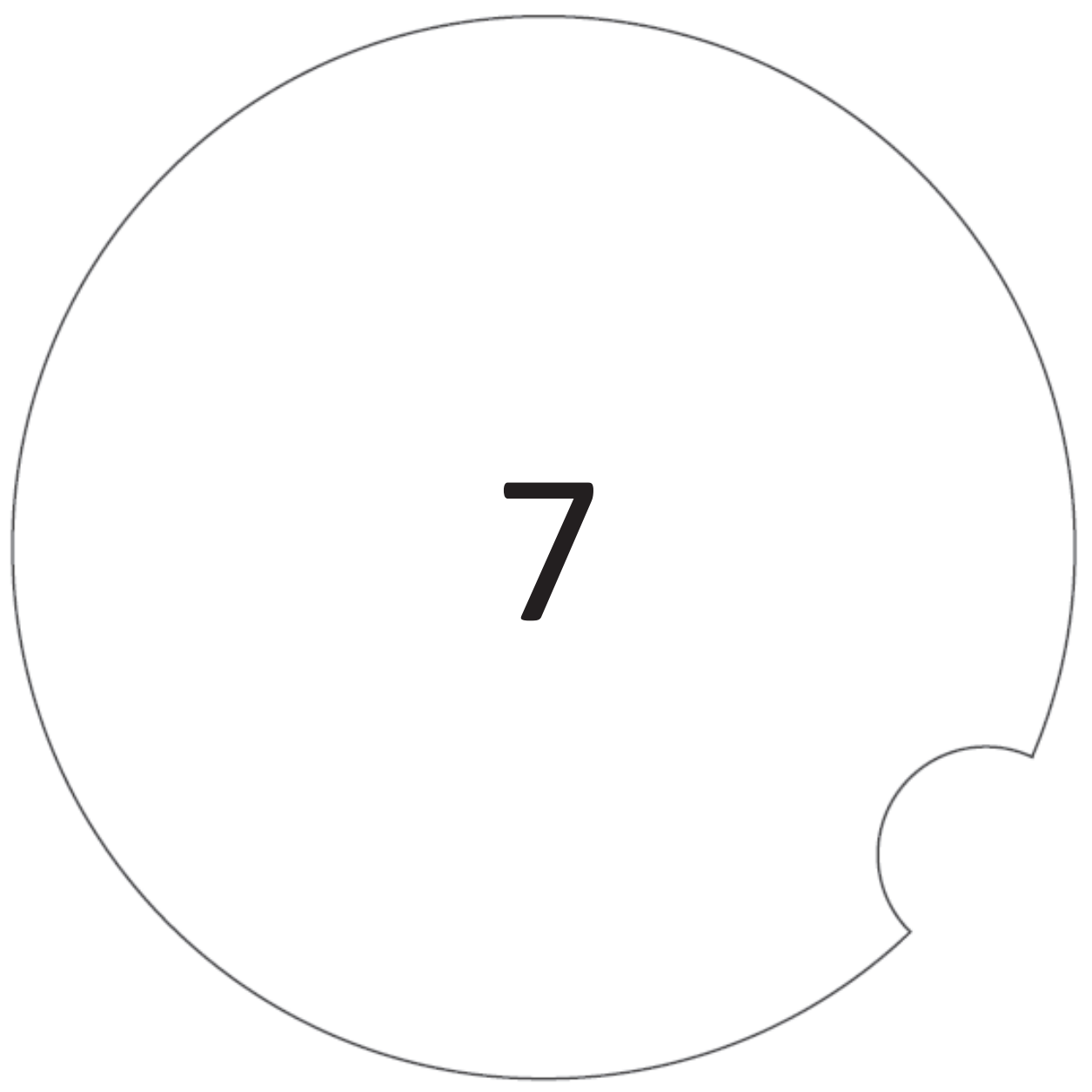

\section{Index event bias - a numerical example}

Smits JM, van Kuijk SM, Leffers P, Peeters LL, Prins MH, Sep SJ Journal of Clinical Epidemiology 2013 Feb. 66(2): 192-6 


\section{Abstract}

Studies of determinants of recurrent disease often give unexpected results. In particular, well established risk factors may seem not to have much influence on recurrence risk. Recently, it has been argued that such paradoxical findings may be due to bias caused by selection of patients based on the occurrence of an earlier episode of the disease. This bias was referred to as index event bias. Here we give a theoretical quantitative example of index event bias, showing that, as a result of selection of patients on the basis of previous disease: (1) risk factors become - inversely associated when they are not in the unselected population, and (2) the crude association between the risk factor of interest and the disease becomes biased toward the null. 


\section{Introduction}

Many medical disorders can recur within individuals. If the risk of developing a disorder is increased after its first occurrence, previously affected individuals constitute a risk group for the disorder and a target for prevention. As prevention can be accomplished by manipulating causal factors, studies have been devoted to identifying causal factors of recurrence and to estimating their strength.

Such studies often give unexpected results. In particular, factors that have been well established as determinants of the first occurrence of a disease may seem not to influence the risk of recurrence much. For instance, factor $V$ Leiden is an established strong risk factor of first-time venous thrombosis, with reported relative risks of up to 80 for homozygous individuals versus non-carriers ${ }^{1}$. However, among patients with a previous thrombotic event, its effect on recurrence is not clear ${ }^{2}$. Another example is that hypertension, while increasing the risk of first-time stroke about fourfold ${ }^{3}$, has turned out as a much weaker risk factor for stroke recurrence, with relative risks ranging from $0.9-1 \cdot 6^{4-8}$.

Dahabreh and $\mathrm{Kent}^{9}$ have recently argued that such paradoxical findings can be the result of the selection of a study population on the basis of the previous occurrence of an event. Due to conditioning on this event, an inverse association between (known and unknown) risk factors may arise, when these risk factors are not mutually associated within the general population. As a consequence, the association between individual risk factors and recurrence of the event will be biased toward the null ("index event bias"). In the example of hypertension and stroke, experimental studies show that reduction of hypertension has strong beneficial effects on stroke recurrence rates $^{10,11}$, further indicating that the apparent weakness of the association in observational studies is indeed a result of bias.

Although Dahabreh and Kent make a strong case for index event bias as an explanation of the paradoxical findings in recurrence risk research, they do not adduce quantitative examples to show the mechanism of the bias. In the present paper we will illustrate the operation of the bias by use of a numerical example.

\section{Simulation in short}

We present hypothetical data for a study intending to measure the association of a particular risk factor with recurrence of a particular pregnancy complication. In order to demonstrate the effects of selection on the basis of a prior complication, we start with a population of first-time pregnant women, let part of them develop the complication, and then focus on second pregnancies among those that developed the complication. 
In the population of first-time gravids, the risk factor of interest is not associated (by design) to other risk factors of the complication. We will demonstrate that, after selection on the basis of prior complication:

1. The risk factor of interest becomes inversely associated with other risk factors of the complication;

2. The crude association between the risk factor of interest and the complication becomes biased toward the null.

\section{Simulation study}

\section{First pregnancy}

Let's consider an initial cohort of 100,000 first-time pregnant women and assume that there are four risk factors involved in the aetiology of the pregnancy complication (C), namely the risk factor of interest (R), two other, unmeasured, risk factors (U1 and U2), and pregnancy $(P)$. We set the marginal frequencies of $R, U 1$, and $U 2$ during the first pregnancy to $0.2,0.2$, and 0.2 , and ensure that the occurrence of each factor is independent of that of each of the other two (table 1). For instance, the likelihood that $\mathrm{U} 1=1$ is 0.20 , whether $\mathrm{R}=1$ or $\mathrm{R}=0$.

\section{Assumptions}

In order to enhance transparency of the example, we make a number of simplifying assumptions, namely:

- All women who experience a pregnancy complication proceed to a next pregnancy;

- Experiencing the complication for the first time does not in itself increase the risk of a new instance of the complication;

- Individual risk profiles (i.e. the combination of risk factors) do not change over the course of the two consecutive pregnancies.

In an additional analysis, we relax the latter assumption by allowing risk factor status to change between the two pregnancies.

Table 1 Joint distribution of risk factors $R, U_{1}$ and $U_{2}$ in 100,000 first-time pregnant women

\begin{tabular}{lrrrrr}
\hline & $U_{1}=1$ & $U_{1}=1$ & $U_{1}=0$ & $U_{1}=0$ & \\
& $U_{2}=1$ & $U_{2}=0$ & $U_{2}=1$ & $U_{2}=0$ & Total \\
\hline$R=1$ & 800 & 3,200 & 3,200 & 12,800 & 20,000 \\
$R=0$ & 3,200 & 12,800 & 12,800 & 51,200 & 80,000 \\
Total & 4,000 & 16,000 & 16,000 & 64,000 & 100,000 \\
\hline
\end{tabular}

Italic numbers indicate women developing pregnancy complication $\mathrm{C}$ because of a combination of risk factors (see table 2 ).

In line with contemporary disease causation theory, we assume that development of disease is the result of the combined action of multiple component causes. We stipulate that causation of $C$ needs the presence of $P$ together with at least two other risk factors. Table 2 lists all combinations of values of risk factors sufficient for $C$ to develop. 
Application of this scheme to our population of primigravids means that 10,400 women (10.4\%) develop the pregnancy complication (see table 1, italic numbers). Risks of developing the complication among individuals with $\mathrm{R}=1$ and $\mathrm{R}=0$ are 0.36 $(7,200 / 20,000)$ and $0.04(3,200 / 80,000)$, respectively, and relative risk (RR) is 9.00 (0.36/0.04).

Table 2 Combinations of risk factors sufficient to cause C. 1 means: risk factor present; 0 means: risk factor absent

\begin{tabular}{cccc}
\hline $\mathbf{R}$ & $\mathbf{U}_{1}$ & $\mathbf{U}_{2}$ & $\mathbf{P}$ \\
\hline 1 & 1 & 0 & 1 \\
1 & 0 & 1 & 1 \\
0 & 1 & 1 & 1 \\
1 & 1 & 1 & 1 \\
\hline
\end{tabular}

\section{Second pregnancy}

In table 3, frequencies of combinations of $R, U_{1}$ and $U_{2}$ are displayed for the second pregnancy among the 10,400 women who experienced the complication in their first pregnancy. Now, the three risk factors have become negatively associated. For instance, if $R=1$, the likelihood that $U_{1}=1$ is 0.56 , while it is 1.00 if $R=0$.

Because only women with a previous complication are included, and no changes in determinant status across pregnancies occur, all women in both strata of $\mathrm{R}$ develop the complication during their second pregnancy, and crude $R R$ for $R=1$ versus $R=0$ is $1.00((7200 / 7200) /(3200 / 3200))$.

Table 3 Joint distribution of risk factors $R, U_{1}$ and $U_{2}$ during the second pregnancy of 10,400 women who developed the pregnancy complication during the first pregnancy

\begin{tabular}{lrrrrr}
\hline & $U_{1}=1$ & $U_{1}=1$ & $U_{1}=0$ & $U_{1}=0$ & Total \\
\hline $\mathrm{R}=1$ & $U_{2}=1$ & $U_{2}=0$ & $U_{2}=1$ & $U_{2}=0$ & 7,200 \\
$\mathrm{R}=0$ & 800 & 3,200 & 3,200 & 0 & 3,200 \\
total & 3,200 & 0 & 0 & 0 & 10,400 \\
\hline
\end{tabular}

Italic numbers indicate women developing recurrent $\mathrm{C}$ because of a combination of risk factors (see table 2).

This value of the (crude) RR is the result of extreme bias toward the null, caused by the introduction of a negative association between $\mathrm{R}$ and the other risk factors. The real (causal) RR can be calculated by means of a counterfactual approach ${ }^{12}$. That is, instead of comparing the observed complication risks of women with $R=1$ and $R=0$, the observed complication risk among women with $R=1$ is compared with the hypothetical risk that would apply if the same women would have $R=0$ instead of $R=1$. Computed in this way, the RR amounts to 9.00 , because its denominator is $0.11(800 / 7200)$ instead of 1.00 (3200/3200). 


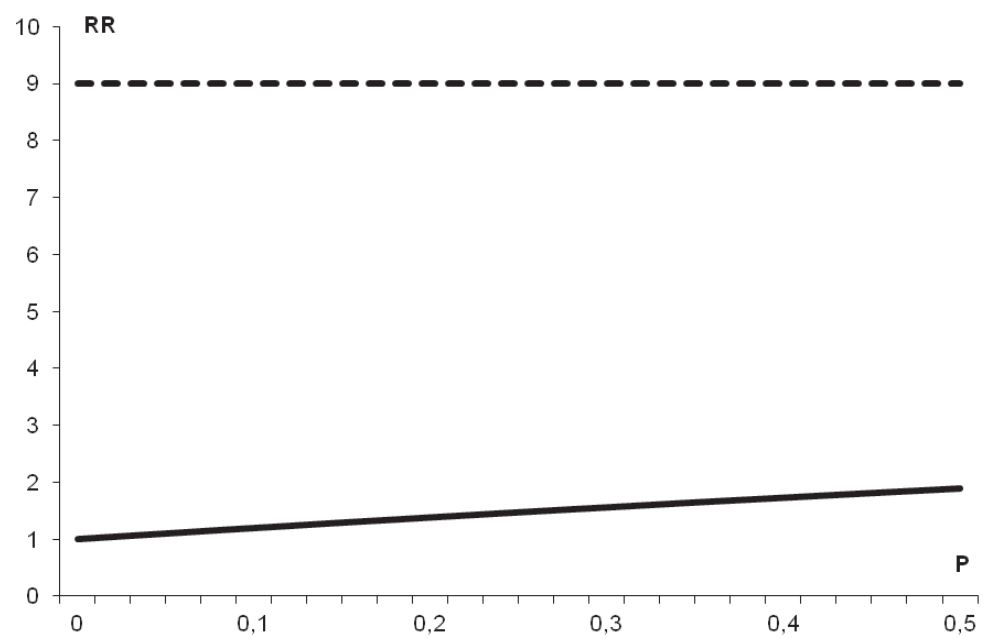

Figure 1 Crude and counterfactual relative risk (RR) for the association between $\mathrm{R}$ and $\mathrm{C}$ during the $2^{\text {nd }}$ pregnancy, as a function of the probability $(P)$ that women with $R=1$ during the $1^{\text {st }}$ pregnancy change to $R=0$ before the start of the $2^{\text {nd }}$. Crude $R R$, solid line; counterfactual $R R$, dashed line

\section{Effect of risk factor change across pregnancies}

In the example above, we did not allow individual risk factor status to change between pregnancies. We carried out some additional calculations to see how such intraindividual changes in the presence of $R, U_{1}$ and $U_{2}$ would influence the amount and direction of bias. Figure 1 displays crude and counterfactual RRs for a simulation in which women exposed to $R=1$ during the first pregnancy can change to $R=0$ before the start of the second pregnancy. Such change would occur, for instance, in case of a preventive intervention on risk factor $R$. When the probability of $R=1$ changing to $R=0$ increases from 0.0 to 0.5 , crude RRs increase from 1 to about 2, while real (counterfactual) RRs remain equal.

Figure 2 shows crude and counterfactual RRs for a simulation in which women with $R=0$ can change to $R=1$, mimicking a situation in which the probability of being exposed to the risk factor increases over time (e.g., advanced age). When the probability of $\mathrm{R}=0$ changing to $R=1$ increases from 0.0 to 0.5 , crude RRs remain 1 , while RRs change from 9.0 to about 3.5 . 


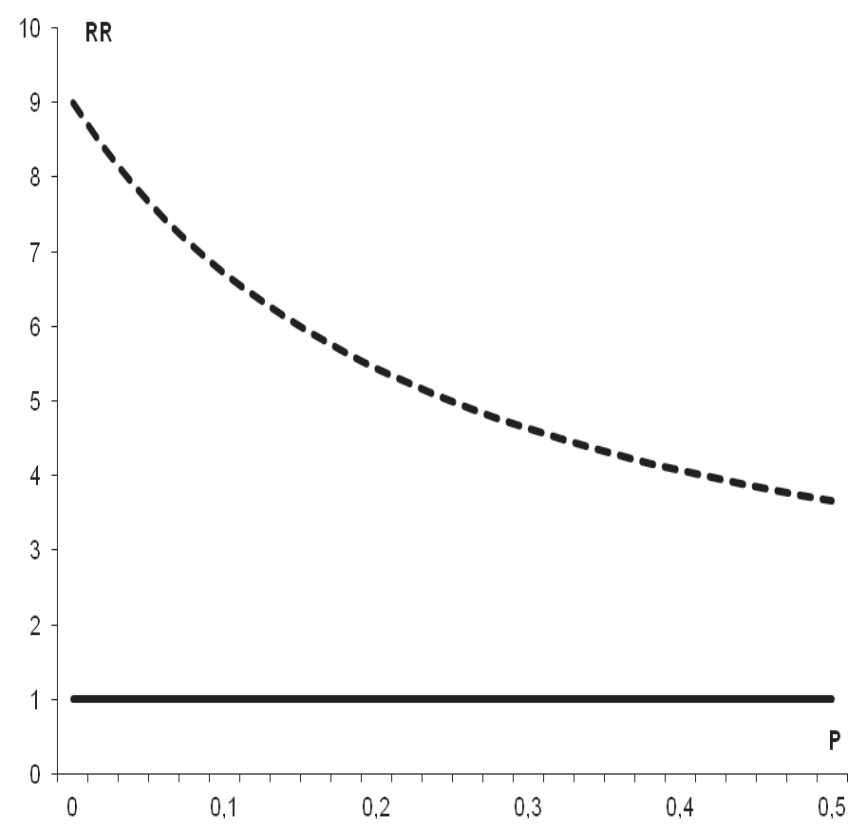

Figure $\mathbf{2}$ Crude and counterfactual relative risk (RR) for the association between $\mathrm{R}$ and $\mathrm{C}$ during the $2^{\text {nd }}$ pregnancy, as a function of the probability $(P)$ that women with $R=0$ during the $1^{\text {st }}$ pregnancy change to $R=1$ before the start of the $2^{\text {nd }}$. Crude RR, solid line; counterfactual RR, dashed line

Figures 3 and 4 show crude and counterfactual RRs for simulations in which women with $U_{1}=1$ can change to $U_{1}=0$ (Figure 3 ) or from $U_{1}=0$ to $U_{1}=1$ (figure 4). These simulations mimic variability of risk factors other than the risk factor of interest. The figures show that transition from $U_{1}=1$ to $U_{1}=0$ leads to an increase of both crude and real RRs, while transition from $U_{1}=0$ to $U_{1}=1$ leads to a decrease in real $R R$ while crude $R R$ remains 1 . Across the observed range of probabilities of transition from $U_{1}=1$ to $\mathrm{U}_{1}=0$ or vice versa, crude and real RRs never cross, and hence bias remains present. 


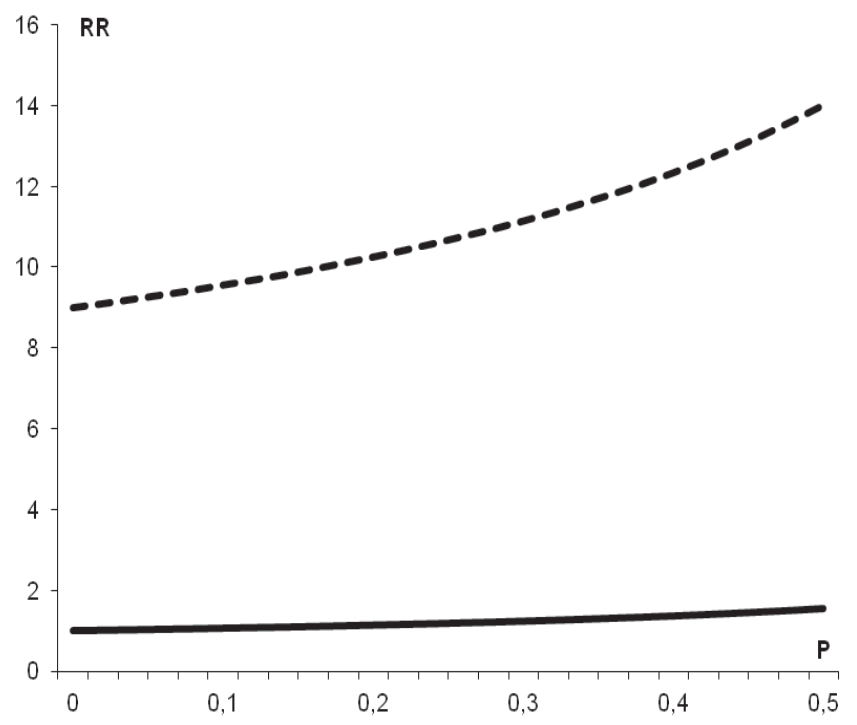

Figure 3 Crude and counterfactual relative risk (RR) for the association between $\mathrm{R}$ and $\mathrm{C}$ during the $2^{\text {nd }}$ pregnancy, as a function of the probability $(P)$ that women with $U_{1}=1$ during the $1^{\text {st }}$ pregnancy change to $U_{1}=0$ before the start of the $2^{\text {nd }}$. Crude RR, solid line; counterfactual RR, dashed line

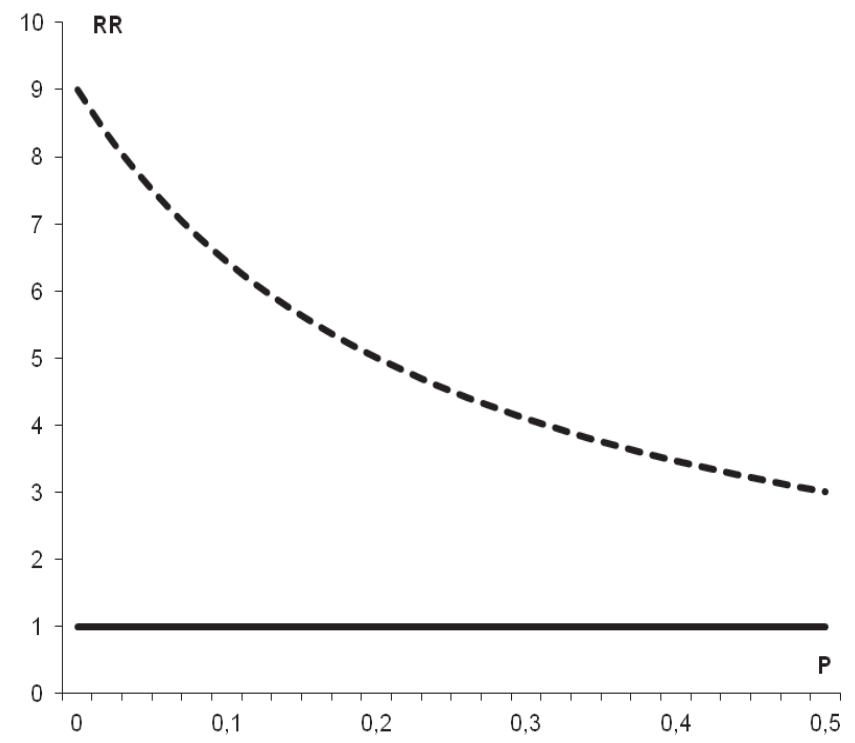

Figure 4 Crude and counterfactual relative risk (RR) for the association between $\mathrm{R}$ and $\mathrm{C}$ during the $2^{\text {nd }}$ pregnancy, as a function of the probability $(P)$ that women with $U_{1}=0$ during the $1^{\text {st }}$ pregnancy change to $U_{1}=1$ before the start of the $2^{\text {nd }}$. Crude RR, solid line; counterfactual RR, dashed line 


\section{Discussion}

Our simulation study illustrates how restriction to individuals with previous disease can lead to biased estimates of the strength of risk factors of disease recurrence. This type of bias has earlier been referred to as 'index event bias' and is a form of 'colliderstratification bias' ${ }^{\prime 13}$. As the bias is attributable to selection of a study population on the basis of a common effect of two (or more) factors, it can be considered a form of selection bias $^{14}$.

The study design considered in this article, in which the study population is restricted to individuals with a first episode of disease, and followed until recurrence of the disease, has frequently been applied in a wide range of medical (and non-medical) disciplines (e.g. references 2,4-8,15-22). Until now, the possibility of index event bias (or collider stratification bias) has not been mentioned in any of these studies, even when the associations that were found were unexpectedly weak. As a consequence, the importance of risk factors of recurrence, and therefore the potential effectiveness of prevention aimed at risk factors may have been underestimated.

In our simulation, we made some simplifying assumptions that merit attention. Firstly, we assumed that all women with the complication in the first pregnancy conceived again. In the real world, a second pregnancy may not occur for a number of reasons. For instance, women with a previous complicated pregnancy may choose to prevent a new pregnancy in order to avoid the risk of a recurrent complication. If the probability of a second pregnancy is unrelated to risk factors of the complication, or only related to the risk factor of interest, the degree of bias will not be different from that in our simulation (since numerator and denominator for the RR do not change). If the probability of a second pregnancy is related to other risk factors of the complication $\left(U_{1}\right.$ or $U_{2}$ in our simulation), the degree of bias may differ from that in the simulation.

Secondly, in our example, we assumed that the pregnancy complication in itself did not affect recurrence risk. This assumption may often not hold, since many disorders, during their first instance, can induce immunity, organ damage, or other long-term effects that may influence risk of recurrence. We chose not to incorporate this effect in our simulations in order to preserve simplicity and focus on the core mechanism of the bias. Further methodological studies could explore effects of adaptations of the model making it comply better with specific real world situations.

It should be noted that the numerical results obtained in our example depend on the particular risk model assumed. The strength of the bias may vary with the number of different sufficient causes and their composition. For a theoretical elaboration of modelling approaches of the effects of conditioning on a collider within a sufficientcomponent causes framework we refer to VanderWeele and Robins ${ }^{23}$. 
Our additional analyses show that the degree of bias decreases when intra-individual variability of the risk factor of interest increases (figures 1-2). On the basis of this result we expect that index-event bias tends to be stronger when unmodifiable factors (such as genetic factors) are studied. We also found that, when intra-individual variability of other (unknown) risk factors increases, the degree of bias can decrease as well as increase (figures 3-4).

In addition to complicating causal research, selection on an index event may affect the accuracy of predictive models of recurrence risk. Because of the introduction of negative associations between measured and unmeasured risk factors, the predictive value of the measured risk factors will decrease. This may lead to the seemingly contradictory finding that a factor proven to be a target for effective recurrence prevention turns out to be a weak predictor of the outcome in a recurrence risk prediction model. More research is needed with respect to the consequences of index event bias for prediction studies.

In our simulation, we used a counterfactual approach to calculate correct relative risks, (i.e. unaffected by index event bias). Obviously this approach is not feasible in the real world, since values of all component causes (as well as their interaction in causing the outcome) need to be known. For now we are not aware of any method by which all bias can be removed in practice. As Darabreh and Kent ${ }^{9}$ have noted, partial control may be achieved by adjusting for important risk factors of the outcome. Residual bias will however always be a concern since generally many causal factors remain unknown.

In conclusion, the possibility of index event bias should be reason for extra caution in the causal interpretation of results of recurrence risk factor studies. The absence of any strong or consistent association of a determinant with recurrence risk should not be reason to discard the factor as a potential focus for preventive action, as exemplified by hypertension and stroke recurrence ${ }^{10,11,16}$. 


\section{References}

1. Rosendaal FR, Koster T, Vandenbroucke JP, Reitsma PH. High risk of thrombosis in patients homozygous for factor $\mathrm{V}$ Leiden (activated protein $\mathrm{C}$ resistance). Blood. 1995 Mar 15;85(6):1504-8.

2. Christiansen SC, Cannegieter SC, Koster T, Vandenbroucke JP, Rosendaal FR. Thrombophilia, clinical factors, and recurrent venous thrombotic events. Jama. 2005 May 18;293(19):2352-61.

3. Sacco RL, Benjamin EJ, Broderick JP, Dyken M, Easton JD, Feinberg WM, et al. American Heart Association Prevention Conference. IV. Prevention and Rehabilitation of Stroke. Risk factors. Stroke. 1997 Jul;28(7):1507-17.

4. Sacco RL, Shi T, Zamanillo MC, Kargman DE. Predictors of mortality and recurrence after hospitalized cerebral infarction in an urban community: the Northern Manhattan Stroke Study. Neurology. 1994 Apr;44(4):626-34.

5. Hankey GJ, Jamrozik K, Broadhurst RJ, Forbes S, Burvill PW, Anderson CS, et al. Long-term risk of first recurrent stroke in the Perth Community Stroke Study. Stroke. 1998 Dec;29(12):2491-500.

6. Petty GW, Brown RD, Jr., Whisnant JP, Sicks JD, O'Fallon WM, Wiebers DO. Survival and recurrence after first cerebral infarction: a population-based study in Rochester, Minnesota, 1975 through 1989. Neurology. 1998 Jan;50(1):208-16.

7. Appelros P, Nydevik I, Viitanen M. Poor outcome after first-ever stroke: predictors for death, dependency, and recurrent stroke within the first year. Stroke. 2003 Jan;34(1):1226.

8. Hillen T, Coshall C, Tilling K, Rudd AG, McGovern R, Wolfe CD. Cause of stroke recurrence is multifactorial: patterns, risk factors, and outcomes of stroke recurrence in the South London Stroke Register. Stroke. 2003 Jun;34(6):1457-63.

9. Dahabreh IJ, Kent DM. Index event bias as an explanation for the paradoxes of recurrence risk research. Jama. Feb 23;305(8):822-3.

10. Friday G, Alter M, Lai SM. Control of hypertension and risk of stroke recurrence. Stroke. 2002 Nov;33(11):2652-7.

11. Rashid P, Leonardi-Bee J, Bath P. Blood pressure reduction and secondary prevention of stroke and other vascular events: a systematic review. Stroke. 2003 Nov;34(11):2741-8.

12. Maldonado G, Greenland S. Estimating causal effects. Int J Epidemiol. 2002 Apr;31(2):4229.

13. Greenland S. Quantifying biases in causal models: classical confounding vs colliderstratification bias. Epidemiology. 2003 May;14(3):300-6.

14. Hernan MA, Hernandez-Diaz S, Robins JM. A structural approach to selection bias. Epidemiology. 2004 Sep;15(5):615-25.

15. MacNeill S, Dodds L, Hamilton DC, Armson BA, VandenHof M. Rates and risk factors for recurrence of gestational diabetes. Diabetes Care. 2001 Apr;24(4):659-62.

16. Egido JA. Benefits of modifying the predictive factors of stroke recurrence. Cerebrovasc Dis. 2005;20 Suppl 2:84-90.

17. Wattanakit K, Folsom AR, Chambless LE, Nieto FJ. Risk factors for cardiovascular event recurrence in the Atherosclerosis Risk in Communities (ARIC) study. Am Heart J. 2005 Apr;149(4):606-12.

18. Burcusa SL, lacono WG. Risk for recurrence in depression. Clin Psychol Rev. 2007 Dec;27(8):959-85.

19. McDonald SD, Best C, Lam K. The recurrence risk of severe de novo pre-eclampsia in singleton pregnancies: a population-based cohort. Bjog. 2009 Nov;116(12):1578-84. 
20. Millet JP, Orcau A, de Olalla PG, Casals M, Rius C, Cayla JA. Tuberculosis recurrence and its associated risk factors among successfully treated patients. J Epidemiol Community Health. 2009 Oct;63(10):799-804.

21. Mohan KM, Crichton SL, Grieve AP, Rudd AG, Wolfe CD, Heuschmann PU. Frequency and predictors for the risk of stroke recurrence up to 10 years after stroke: the South London Stroke Register. J Neurol Neurosurg Psychiatry. 2009 Sep;80(9):1012-8.

22. Salvatore S, Athanasiou S, Digesu GA, Soligo M, Sotiropoulou M, Serati $M$, et al. Identification of risk factors for genital prolapse recurrence. Neurourol Urodyn. 2009;28(4):301-4.

23. VanderWeele TJ, Robins JM. Directed acyclic graphs, sufficient causes, and the properties of conditioning on a common effect. Am J Epidemiol. 2007 Nov 1;166(9):1096-104. 


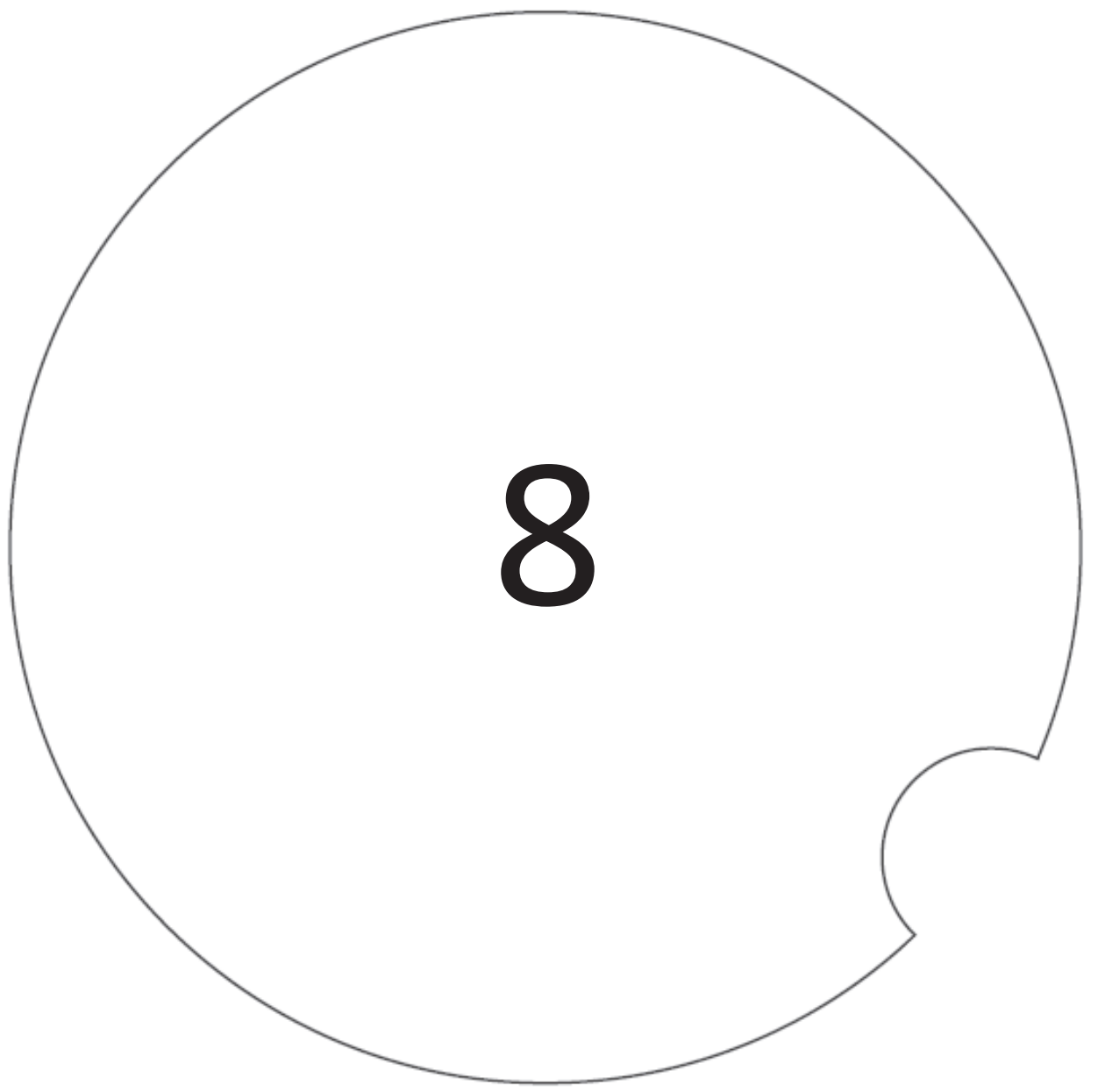

Bias in regression coefficient estimates when assumptions for handling missing data are violated

van Kuijk SM, Peeters LL, Viechtbauer W, Smits LJ Submitted 


\section{Abstract}

\section{Objective}

It is common that researchers are unclear about handling missing data. In this paper, the authors will show that violation of assumptions using either multiple imputation or complete case analyses may introduce bias.

\section{Study design}

The authors performed a simulation to assess the performance of Complete Case (CC) analysis and Multiple Imputation (MI) with different missing data mechanisms (missing completely at random (MCAR), at random (MAR), and not at random (MNAR)). The study focused on the point estimation of regression coefficients and standard errors.

\section{Results}

When data were MAR conditional on $\mathrm{Y}, \mathrm{CC}$ analysis resulted in biased regression coefficients; they were all underestimated in our scenarios. In these scenarios, analysis after MI gave correct estimates. Yet, in case of MNAR MI yielded biased regression coefficients, while CC analysis performed well.

\section{Conclusion}

The authors demonstrated that MI was only superior to CC analysis in case of MCAR or MAR. In some scenarios CC may be superior over MI. Often it is not feasible to identify the reason why data in a given dataset are missing. Therefore, more emphasis should be put on reporting the extent of missing values, the method used to address them, and the assumptions that were made. 


\section{Introduction}

One of the most ubiquitous problems in epidemiological studies is that of missing values. For instance, certain lab results may be missing in subjects from a specific hospital because of local protocol, or not all participants completed personal questions in a survey. In follow-up studies, people who drop out of the study, who have passed away, or who have moved away to some unknown destination can result in whole questionnaires missing. The standard approach to handling missing values for most statistical packages, and therefore the easiest method to implement is to omit subjects with missing data on one or more of the variables used for the analysis. This method is called complete case (CC) analysis and can result in a considerable loss of statistical power because fewer subjects are included in the analysis than planned. Moreover, CC analysis may cause bias in the parameter estimates ${ }^{1-4}$. Yet an important advantage of this method is that no missing values are filled in by values that were not observed, which are therefore always uncertain.

The extent of the problem of missing values has been widely acknowledged for decades by statisticians and many methods to deal with it have been developed since. Most methods aim to impute plausible values where data are missing, providing the analyst with a completed dataset. These methods also make specific assumptions about the mechanism(s) that caused the data to be missing. An increasingly popular imputation method among researchers is Multiple Imputation (MI) ${ }^{5-7}$. This method creates multiple completed datasets, which can be analysed with standard statistical methods. As it is often implemented in practice, $\mathrm{Ml}$ gives valid results when data are missing conditional on the value of other variables in the imputation model, including the outcome variable, which is called Missing at Random (MAR) ${ }^{8}$. For circumstances in which the MAR assumption is met, or when data are truly missing at random (missing completely at random, MCAR), MI has been shown to be superior to other popular imputation methods such as imputation with the (conditional) mean, last observation carried forward, and single regression imputation. It yields unbiased parameter estimates and gives standard errors that are corrected for the uncertainty introduced by the imputation ${ }^{2-4,9}$.

The use of any method of handling missing data can introduce bias if the missing data assumptions are not met. However, it is not customary that such methods are reported in detail, and if they are, the authors usually do not provide convincing support for the validity of the assumptions ${ }^{10}$. A third and more problematic missing data mechanism is often overlooked, namely Missing Not at Random (MNAR), in which values are missing conditional on their own true value or the value of covariates that are unmeasured.

In a recent simulation study, White and Carlin ${ }^{11}$ showed that when data are not MCAR, there are some scenarios under which a CC analysis yields negligible bias while MI can introduce considerable bias into the coefficients of a regression model. They also provide an index for assessing the likely gain in precision from $\mathrm{MI}$. These findings raise some doubt about the routine application of $\mathrm{MI}$ as a panacea for dealing with missing 
data. In the present article, we focus further on the performance of $\mathrm{MI}$ as compared to CC analysis in scenarios when assumptions are violated, with particular emphasis on scenarios that have received little attention in other simulation studies. In addition, we assessed what parameters of the regression model determined the extent of the bias and whether they influence the sign of bias on the regression coefficients. We focus in particular on $\mathrm{Ml}$ as the imputation method, since it is commonly considered to be superior to other popular imputation methods. To compare MI with CC analysis, we performed a Monte Carlo simulation study based on a linear regression model, with two independent variables, one of which was incompletely observed.

\section{Methods}

\section{Study design}

We performed a Monte Carlo simulation study to evaluate the properties of MI and CC in several missing data scenarios. We simulated data based on a linear model given by

$$
Y=\beta_{0}+\beta_{1} X_{1}+\beta_{2} X_{2}+\varepsilon
$$

were $Y$ is the outcome variable, $X_{1}$ and $X_{2}$ are the independent variables, $\beta_{0}$ is the intercept, and $\beta_{1}$ and $\beta_{2}$ denote the regression coefficients for the corresponding $X$ variables, and $\varepsilon$ is the error variable (or residual). The independent variables were drawn from a multivariate normal distribution with zero means, unit variances, and correlation equal to $\rho$. The errors were drawn from an independent normal distribution with zero mean and variance equal to $\sigma^{2}$. The model intercept, $\beta_{0}$, was set to 0 . To determine the influence of sample size $(n)$, the error variance $\left(\sigma^{2}\right)$, the correlation between the independent variables $(\rho)$, and the size of the regression coefficients relative to one another $\left(\beta_{1}\right.$ and $\left.\beta_{2}\right)$, we varied all these factors throughout the simulation study. Table 1 shows all the values considered for the different simulations. This amounts to 315 different conditions.

Table 1 Variables that were varied between simulations and the values that were considered

\begin{tabular}{|c|c|c|c|c|c|c|c|}
\hline \multirow[b]{2}{*}{ Sample size $(n)$} & \multicolumn{7}{|c|}{ Values considered for simulation } \\
\hline & 100 & 500 & 1000 & & & & \\
\hline Error variance $\left(\sigma^{2}\right)$ & 0.5 & 1 & 2 & 4 & 8 & & \\
\hline Correlation between $X_{1}$ and $X_{2}(\rho)$ & -0.75 & -0.50 & -0.25 & 0.00 & 0.25 & 0.50 & 0.75 \\
\hline Coefficient for $X_{1}\left(\beta_{1}\right)^{1}$ & 0.5 & 1 & 2 & & & & \\
\hline Coefficient for $X_{2}\left(\beta_{2}\right)^{1}$ & 2 & 1 & 0.5 & & & & \\
\hline Proportion missing data ( $\pi)$ & 0.25 & 0.5 & 0.75 & & & & \\
\hline
\end{tabular}

${ }^{1}$ Not all possible combinations of the regression coefficients were considered, only ( $\beta_{1}$ and $\beta_{2}$ respectively) 0.5 and 2,1 and 1 , and 2 and 0.5 . 


\section{Creation of missing values}

We created missing values on the $X_{1}$ variable according to the MCAR, MAR, and the MNAR mechanisms. For the MCAR mechanism, we drew a number from a Bernoulli distribution for each $X_{1}$ value, the probability of success being the same as the proportion of values missing for that experiment. For each successful trial, the corresponding $X_{1}$ value was set to missing. The MAR mechanism was divided into MAR conditional on variable $X_{2}$ (i.e. the higher the $X_{2}$ value, the larger the probability that the corresponding $X_{1}$ variable was missing), and MAR conditional on $Y$ (i.e., the higher the $Y$ value, the larger the probability that the corresponding $X_{1}$ variable was missing). To create missing values according to the first, we drew a normally distributed random variable with a standard deviation of 1 , and compared its value to the corresponding $X_{2}$ value. If it was lower than that value, the corresponding $X_{1}$ value was deleted. The latter mechanism was created by comparing a similar random draw to the corresponding $\mathrm{Y}$ value. The mean of both normal distributions was set for each scenario so that the required proportion of values missing was met. To simulate a MNAR mechanism, the probability of a value being missing was associated with its own value (i.e., the higher the value, the higher the probability of being missing). This was achieved in a similar way to both MAR mechanisms, only this time a random draw from a normally distributed variable, with a mean value chosen to create the desired proportion of missing values and a standard deviation of 1 , was compared to the corresponding $\mathrm{X}_{1}$ value itself. In case the random draw was lower, the corresponding $\mathrm{X}_{1}$ value was deleted. These 4 mechanisms were applied separately to all 315 conditions. Furthermore, we varied the proportion of missing values in all datasets $(\pi)$. We created missing values in proportions of $0.25,0.50$, and 0.75 . Therefore, a total of $315 * 4 * 3=$ 3.780 different conditions were examined in the simulation study.

\section{Data analysis}

We analysed all these scenarios using both CC analysis and standard analysis after MI. We computed the mean of the estimated the regression coefficients $\beta_{0}, \beta_{1}$ and $\beta_{2}$. MI was performed using the mice package (Multiple Imputation using Chained Equations), developed by the Netherlands Organization for Applied Scientific Research (TNO) ${ }^{12}$. The default settings were used, which means that the imputations were generated using the frequently employed predictive mean matching method, and the number of multiple imputations was set to 5 . To make sure that our results could not be attributed to selecting the default method, we reran all scenarios in which bias occurred but this time creating imputations based on the linear regression model. All simulations were performed 1,000 times to obtain stable estimates of regression coefficients and standard errors. $\mathrm{R}^{13}$ was used for the generation of the data and analyses. 


\section{Results}

Table 2 shows the mean of the parameter estimates and corresponding standard errors for the complete data and when applying a CC analysis and analysis after $\mathrm{MI}$ for all considered missing data mechanisms.

Table 2 Mean of the regression coefficient estimates (and corresponding standard errors) for $\mathrm{n}$ $=1000, \sigma^{2}=1, \beta_{1}=\beta_{2}=1, \rho=0.00$, and $\pi=0.5$

\begin{tabular}{lcccccc}
\hline & \multicolumn{2}{c}{ Complete Case Analysis $(\mathrm{CC})$} & \multicolumn{3}{c}{ Multiple Imputation (MI) } \\
\hline & $\beta_{0}$ & $\beta_{1}$ & $\beta_{2}$ & $\beta_{0}$ & $\beta_{1}$ & $\beta_{2}$ \\
Complete data & 0.00 & 1.00 & 1.00 & 0.00 & 1.00 & 1.00 \\
& $(0.032)$ & $(0.032)$ & $(0.032)$ & $(0.032)$ & $(0.032)$ & $(0.032)$ \\
MCAR & 0.00 & 1.00 & 1.00 & 0.00 & 1.00 & 1.00 \\
& $(0.045)$ & $(0.045)$ & $(0.045)$ & $(0.039)$ & $(0.036)$ & $(0.040)$ \\
MAR conditional on X2 & 0.00 & 1.00 & 1.00 & 0.00 & 1.00 & 1.00 \\
& $(0.054)$ & $(0.045)$ & $(0.054)$ & $(0.046)$ & $(0.036)$ & $(0.046)$ \\
MAR conditional on $\mathrm{Y}$ & -0.59 & 0.77 & 0.77 & 0.02 & 0.99 & 1.00 \\
& $(0.048)$ & $(0.044)$ & $(0.044)$ & $(0.042)$ & $(0.039)$ & $(0.043)$ \\
MNAR conditional on X1 & 0.00 & 1.00 & 1.00 & 0.39 & 1.11 & 1.00 \\
& $(0.054)$ & $(0.054)$ & $(0.045)$ & $(0.044)$ & $(0.046)$ & $(0.041)$ \\
\hline
\end{tabular}

\section{Complete case analysis}

Compared to the results generated for the complete data, standard errors increase with increasing loss of data. When the MCAR assumption holds, regression coefficients are unbiased. Moreover, when data are MAR, conditional on $X_{2}$, or when data are MNAR, conditional on its own value, CC also yields unbiased regression parameter estimates. Only when data are MAR, conditional on the outcome variable, does CC result in biased estimates. Both regression coefficients are underestimated, with the intercept being overestimated.

\section{Multiple Imputation}

When data are MCAR or MAR, which is an assumption of MI, analysis yields unbiased parameter estimates. The standard errors are slightly higher than those of the complete data, because the uncertainty of imputation must be taken into account. When the MAR assumption is violated (i.e., MNAR), analysis after MI overestimates the regression coefficient of $X_{1}$ and the intercept.

\section{Influence of model parameters}

The following results describe conditions in which the sample size $\mathrm{n}$ was 1000 , and, unless otherwise stated, the true values of the regression coefficients $\beta_{1}$ and $\beta_{2}$ were 1 , the error variance was 1 , the proportion of missing values was 0.5 , and the correlation between the independent variables was 0.00 .

\section{CC analysis when data are MAR conditional on $Y$}

Figure $1(\mathrm{a}-\mathrm{f})$ shows the influence of model parameters on scenarios in which CC yielded biased results, namely when data were missing at random, conditional on the 
dependent variable $\mathrm{Y}$. When the intercept is considered, for instance in prediction research, Figures $1 \mathrm{a}$ and $1 \mathrm{~b}$ illustrate that the size of bias on the estimation of the intercept is greatly influenced by the variance in the error term and consequently the variance on $Y$. Furthermore, the size of bias is proportional to the proportion of missing values on $X_{1}$. The amount of correlation between the two predictor variables as well as and the true values of the regression coefficients have no influence on the bias of the mean intercept estimate.

Figures $1 \mathrm{c}$ and $1 \mathrm{~d}$ show the bias in the estimate of the regression coefficient of $\mathrm{X}_{1}$ (i.e., $\beta_{1}$ ). The same conclusions hold as for the estimation of the intercept, but now the correlation between the independent variables $X_{1}$ and $X_{2}$ also influences the bias. The bias is highest with a high negative correlation, and lowest with a high positive correlation. The relation seems approximately linear. Figure 1d illustrates that the bias depends on the true value of the regression coefficient and is in fact proportional to its size.

Figures $1 \mathrm{e}$ and $1 \mathrm{f}$ show the results for the estimate of the regression coefficient $\beta_{2}$. For both graphs, the same conclusions can be drawn as for the estimation of the regression coefficient for $X_{1}$. In our scenarios considering MAR, conditional on the dependent variable $\mathrm{Y}, \mathrm{CC}$ analysis always yielded an underestimation of the regression coefficients and a negative bias in the estimation of the regression coefficients. 

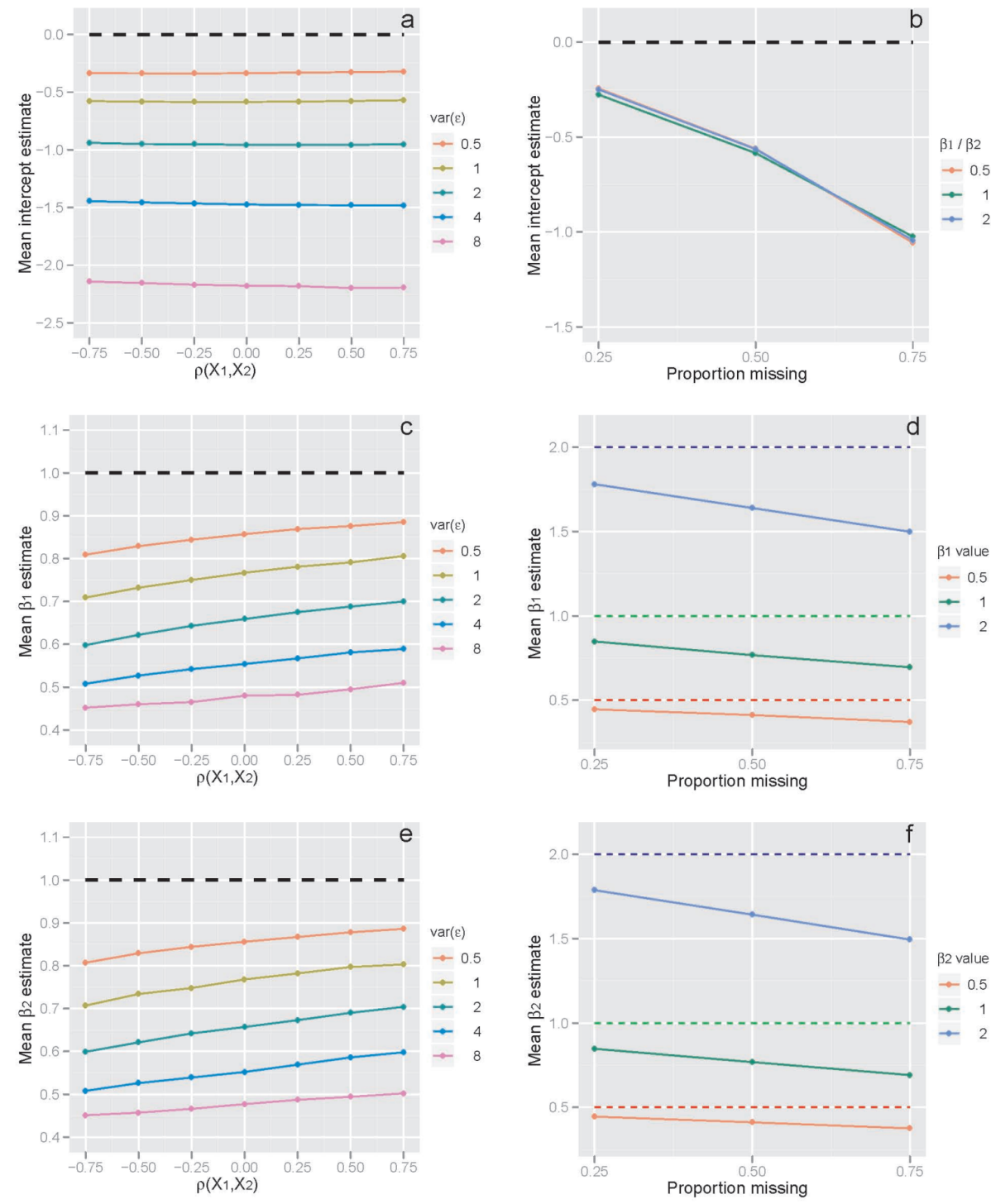

Figure 1(a-f) The mean estimated regression coefficients using CC analysis when data are missing conditional on the outcome variable $Y$, i.e. MAR conditional on $Y$. a, $c$ and e show the influence of the error variance and the correlation between the independent variables; $b, d$ and $f$ show the influence of the true value of the coefficients $\beta_{1}$ and $\beta_{2}$ and the proportion of missing values on $\mathrm{X}_{1}$ 
Analysis after MI when data are MNAR conditional on $X_{1}$

Figure 2(a-f) shows the influence of model parameters on scenarios in which analysis after $\mathrm{MI}$ yielded biased results, namely when data were missing not at random, conditional on the true value of $X_{1}$. Figures $2 a$ and $2 b$ show the results for the model intercept, revealing that the bias is dependent on the error variance (and hence, the variance of $Y$ ), the correlation between $X_{1}$ and $X_{2}$, the proportion of missing values on $X_{1}$, and the size of the regression coefficients relative to one another. The bias increases as a function of the variance of the error term. The weaker the correlation between the independent variables is, the larger the bias in the estimation of the intercept will be. This relationship can be described by a curve (see figure 2a). Just as in the previous examples with CC analysis, the bias increases as a function of the proportion of values missing. Contrary to results from the CC analysis is that now the true value of the regression coefficients also influences bias on the estimation of the intercept.

Figures $2 c$ and $2 d$ show bias in the estimation of the regression coefficient of $X_{1}$. The results are similar to those of the estimation of the intercept. However, now there is an inverse relation between error variance and magnitude of the bias. It seems that the lower the variance is, the higher is the bias. Furthermore, instead of an overestimation of the regression coefficient, scenarios with a large error variance and a strong correlation between the independent variables show a slight underestimation of the regression coefficient. Figure $2 \mathrm{~d}$ shows that there is an overestimation of the regression coefficient, dependent on the true value, while the bias is not that much affected by the proportion of missing values.

Figures $2 \mathrm{e}$ and $2 \mathrm{f}$ illustrate bias in the estimation of the regression coefficient for $\mathrm{X}_{2}$ when data are missing not at random. Bias increases as a function of error variance. In these scenarios, it is evident that the coefficient will be underestimated in case of a negative correlation between $X_{1}$ and $X_{2}$, and overestimated in case of a positive correlation. The estimation of this coefficient seems hardly influenced by the true value of the coefficient. In all conditions, the relative size of the bias on the estimated regression coefficients was not affected by sample size (data not shown).

We replicated all scenarios in which regression coefficients were biased using the linear regression model to predict imputations for missing $X_{1}$ values. In all instances, the occurrence and sign of bias was conform our prior results, and the influence of changes in model parameters remained the same (data not shown). 


\section{Discussion}

Our simulations indicate that estimated regression coefficients for incomplete data can be biased using a standard approach like CC, but also when using a sophisticated method like MI. When data are MCAR or MAR, conditional on $X_{2}$, both methods perform well, although a larger loss of precision when using CC can be observed because of the omission of subjects, which holds for all missing data conditions we analysed. In the conditions we analysed, CC only yielded biased regression coefficients when missing data were MAR conditional on $Y$. Moreover, when data were MNAR, results obtained after $\mathrm{MI}$ were biased whereas CC yielded correct estimates. In scenarios where CC analysis leads to biased results, the regression coefficients were underestimated. Conversely, in scenarios where analysis after MI yielded biased results, the regression coefficients were in most cases overestimated. In most of the scenarios we considered, the magnitude of the bias was influenced by the error variance, or the variance of $Y$, by the correlation between the independent variables, the proportion of missing values, and the true value of the regression coefficients $B_{1}$ and $B_{2}$.

Relatively little attention has been given to MNAR in other empirical or simulation studies. It is described as unlikely to occur ${ }^{14}$, or described as a scenario for which no universal method can provide reliable regression estimates ${ }^{4,8,14,15}$. A prime example of data MNAR is the following. Consider a personal question in a survey which people with a particular value or answer are less likely to complete, for instance asking smoking status among pregnant women. Women who still smoke during pregnancy are more likely to skip the question than women who do not smoke. Another example is asking for income or salary, a question less likely to be completed by people with very low or high incomes. When visiting a gynaecologist during pregnancy, body mass index (BMI) may be recorded only when it is suspected to be relevant, that is either very low or very high. For clinical laboratory results, MNAR may occur when a ceiling effect is present, that is, when a level above which a variable can be measured is reached, and is therefore not observed.

Of course, many more real-data examples can be produced. It follows from our results that determining the missing data mechanisms are crucial for choosing the correct method for handling missing values. Yet it is hard, if not impossible to demonstrate empirically that one mechanism in particular is responsible for the missing data or to rule out another. It can only be assumed after detailed inspection of the raw data and considering thoroughly the data collection procedure. Next, despite the fact that the missing data mechanisms are usually presented as mutually exclusive, it seems likely in many cases that, in any given large multivariable dataset, combinations of mechanisms can be the reason for incomplete data, presenting the analyst with an even more difficult problem when deciding what method to use. Furthermore, for imputation methods based on predictions using other covariates, enough relevant covariates should be present in the dataset. As a consequence, imputation methods may perform worse in empirical datasets than has been shown in simulation studies performed under perfect circumstances. 

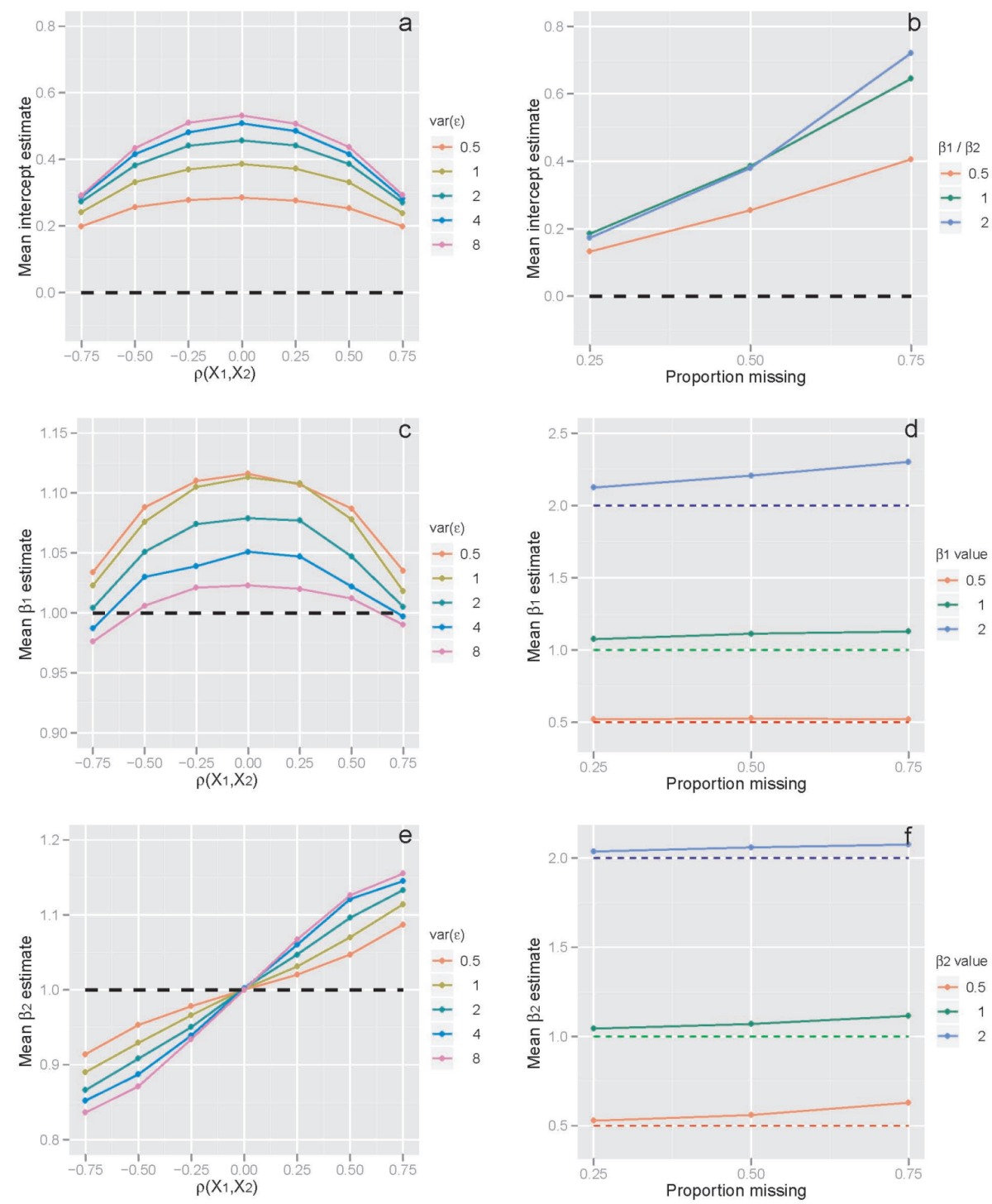

Figure 2(a-f) The mean estimated regression coefficients after MI when data are MNAR conditional on $X_{1}$. a, c and e show the influence of the error variance and the correlation between the independent variables; $b, d$ and $f$ show the influence of the true value of the coefficients $\beta_{1}$ and $\beta_{2}$ and the proportion of missing values on $X_{1}$. To assess the influence of the $\beta_{2}$ value and the proportion of missing values on the mean $\beta_{2}$ estimate (figure $2 f$ ), we set $\rho$ $=0.50$, since figure $2 \mathrm{e}$ shows no bias when $\rho=0.00$ 
One important drawback of our study is that there are many more theoretical scenarios that can be simulated using some sort of MNAR mechanism, which falls well beyond the scope of this article. We emphasize that, since these were not simulated, we cannot generalize our results to other scenarios. This also holds for scenarios in which the model is expanded with more covariates, interaction terms, multilevel structure, or other features. Our goal was to present a simple simulation for illustrative purposes and discussing many more missing data mechanisms is unlikely to alter our conclusion. Another drawback is that, in order for missing values to be imputed, they first have to be made missing. In all simulations, missing values are created according to known, predefined, mechanisms. In empirical research, the data analyst starts with an incomplete dataset, often with little information on the causes. Therefore, it is unknown how well these simulation studies reflect real-life analysis problems.

An important improvement could be if authors of empirical articles put much more emphasis on describing the extent of the problem of missing values, the methods used to handle it, and the assumptions that were made about the mechanism(s) that contributed to the occurrence of missing values. To this end, Sterne et al. ${ }^{16}$ propose guidelines for reporting results of data affected by missing data. This has the potential to provide the interested reader with sufficient information to determine the validity of the results in the context of the research question addressed. In addition to these guidelines, authors could assess differences in results between a CC analysis and analysis after $\mathrm{Ml}$ to give insight in the stability of the results using different methods. However, even now it is important to keep in mind that similar results do not guarantee unbiased estimates.

In conclusion, this paper does not intend to discourage the use of $\mathrm{MI}$ or CC for that matter. However, we intended to demonstrate the importance of keeping in mind that the routine application of $\mathrm{MI}$ can have some pitfalls, while CC may give more valid results in some scenarios. As a result, some uncertainty is always introduced when missing values are present. 


\section{References}

1. van der Heijden GJ, Donders AR, Stijnen T, Moons KG. Imputation of missing values is superior to complete case analysis and the missing-indicator method in multivariable diagnostic research: a clinical example. J Clin Epidemiol. Oct 2006;59(10):1102-1109.

2. Allison PD. Missing Data. lowa: Sage; 2001.

3. Steyerberg EW. Clinical Prediction Models. New York: Springer; 2009.

4. Harrell Jr FE. Regression Modeling Strategies. New York: Springer; 2001.

5. Rubin DB. Multiple imputations in sample surveys: a phenomenological \{Bayesian\} approach to nonresponse. Paper presented at: Proceedings of the Survey Research Methods Section1978.

6. Rubin DB. The Design of a General and Flexible System for Handling Nonresponse in Sample Surveys. The American Statistician. 2004/11/01 2004;58(4):298-302.

7. Schafer JL. Multiple imputation: a primer. Stat Methods Med Res. Mar 1999;8(1):3-15.

8. Rubin DB. Inference and missing data. Biometrika. December 1, 1976 1976;63(3):581-592.

9. Janssen KJ, Vergouwe $\mathrm{Y}$, Donders $A R$, et al. Dealing with missing predictor values when applying clinical prediction models. Clin Chem. May 2009;55(5):994-1001.

10. Fernandes-Taylor S, Hyun JK, Reeder RN, Harris AH. Common statistical and research design problems in manuscripts submitted to high-impact medical journals. BMC Res Notes.4:304.

11. White IR, Carlin JB. Bias and efficiency of multiple imputation compared with completecase analysis for missing covariate values. Stat Med. Dec 10;29(28):2920-2931.

12. van Buuren $\mathrm{S}$, Oudshoorn CGM. Multivariate Imputation by Chained Equations. MICE V1.0 User's manual. TNO report PG/VGZ/00.038. 2000.

13. Duckitt K, Harrington D. Risk factors for pre-eclampsia at antenatal booking: systematic review of controlled studies. Bmj. Mar 12 2005;330(7491):565.

14. Schafer JL. Analysis of incomplete multivatiate data. London: Chapman \&Hall/CRC Press; 1997.

15. Donders AR, van der Heijden GJ, Stijnen T, Moons KG. Review: a gentle introduction to imputation of missing values. J Clin Epidemiol. Oct 2006;59(10):1087-1091.

16. Sterne JA, White IR, Carlin JB, et al. Multiple imputation for missing data in epidemiological and clinical research: potential and pitfalls. BMJ. 2009;338:b2393. 


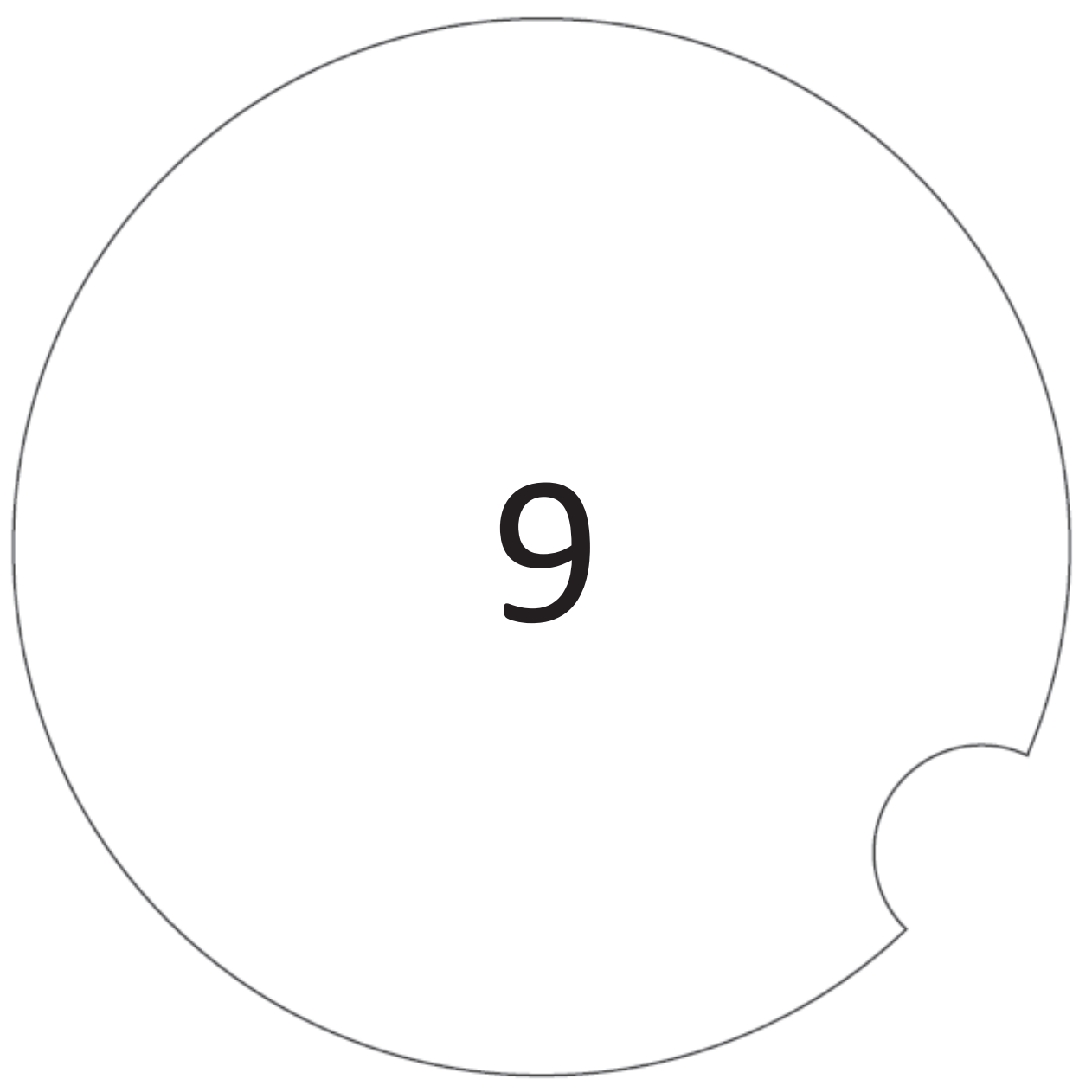

Summary and general discussion 
This thesis comprises an empirical and a methodological part. In the empirical part (Part I) we describe the development, external validation, and impact evaluation of a prediction model for recurrent early-onset preeclampsia (Chapters 2, 4, and 5, respectively, with Chapter 3 describing the design of the PreCare study). In the methodological part (Part II) we report on three theoretical studies relevant to prediction research (Chapters 6, 7, and 8). In the following text, we will summarize the background, design and results of the studies contained in part I, discuss their implications and limitations, and give some recommendations for further research. Next, we will discuss the findings and implications of the methodological studies of part II.

\section{Part 1: Prediction of recurrent early-onset preeclampsia}

\section{Background}

In the Netherlands, we have guidelines for the antenatal care for pregnant women diagnosed with a hypertensive disorder of pregnancy (such as pregnancy-induced hypertension, PE, and the HELLP-syndrome) ${ }^{1}$. These guidelines contain recommendations for the use of antihypertensive drugs, corticosteroids, the prophylactic use of magnesium sulphate, and counselling about the prognosis of the neonate. In addition, regularly-updated guidelines are available for the antenatal care for women at increased risk for maternal and/or foetal morbidity or mortality in conjunction with a certain pre-existing medical condition such as chronic hypertension, diabetes mellitus, renal disease, and obesity, as these conditions are associated with an increased risk of developing placental insufficiency with or without a hypertensive pregnancy disorder, often requiring premature termination of pregnancy. Interestingly, there are no guidelines for the prenatal care of women with a history of hypertensive pregnancy disorder, although the risk of hypertensive complications in a next pregnancy is at least 3 -fold as high in these women as compared to healthy nulliparous women ${ }^{2,3}$.

The lack of a standard management directive for these former patients has resulted in differences between hospitals in the care provided to these women ${ }^{4}$. Instead, more evidence-based differences between patient management could be guided directly by the recurrence risk of the patient. We therefore aimed to develop and validate a prediction model that could be used for recurrence-risk stratification of pregnant women with a history of early-onset preeclampsia. In this context, it is relevant that recurrence risk prediction based on observational or experimental evidence is often more accurate than deduced from clinical judgment only ${ }^{5}$.

Development and external validation of a model for recurrent early-onset PE or HELLP We developed a model to predict recurrent early-onset PE, including the HELLPsyndrome, based on predictor variables that could be routinely obtained after a first complicated pregnancy (Chapter 2). We chose 5 predictors that were reported to have an association with recurrence risk, the selection being based on their preconceptional availability in our combined cohorts together with the opinions of the expert- 
obstetricians participating in this study. The following five predictor variables were selected: fasting glucose levels, previous pregnancy duration, previous SGA baby, presence of chronic hypertension and BMI. These variables were entered in a logistic regression model, which was internally validated using bootstrapping techniques and was evaluated on properties of discriminative ability and calibration. It showed reasonable discriminative ability and was well calibrated on the derivation data. Although many studies evaluated risk factors for PE recurrence ${ }^{6-11}$, and a prepregnancy model had been developed to predict recurrence up to 37 weeks gestation $^{12}$, our model was the first designed specifically to predict PE recurrence, requiring delivery for medical reasons before 34 weeks.

In Chapter 4 we described the external validation of the model using a subset of women from the PreCare data who gave birth before 34 weeks in their previous complicated pregnancy. In this cohort of women originating from different geographical areas in the Netherlands, the performance of the model -as reflected in an area-under-the-ROC curve (AUC)- was only 59\%, which was less than that observed in the internal validation (65\%). This indicates poor ability to discriminate between women who will and who will not develop recurrent early-onset disease in their next pregnancy. Besides, evaluation of the model's calibration measures indicated that, on average, the calculated risk systematically underestimated the observed risk over the entire range of predicted probabilities.

\section{Discussion of the findings}

A number of possible reasons may have contributed to the sharp decline in performance of the prediction model when it was applied to a new cohort. Three potential causes will be elaborated upon here.

Firstly, we noticed much uncertainty in the estimation of model parameters, as reflected by the standard errors. Inspection of the confidence intervals reveals that in some cases the association of predictor variables with the log-odds of recurrence might as well be inversed in reality. The direction of the regression coefficient for both predictor variables "fasting level of glucose" and "birth of an SGA infant" was contrary to what seemed clinically plausible. This may be due to sampling error and as a consequence, it may have rendered the model externally invalid. Nonetheless, it is not justifiable to omit these variables from the model simply because of their counterintuitive association with recurrence risk. Since we aimed to develop a model for disease recurrence rather than occurrence, it is possible that we introduced indexevent bias ${ }^{13}$. By only using data of women with a history of PE to build the model, the relation between established risk factors and outcome may have become weaker or may even have reversed ${ }^{13}$. If indeed index-event bias led to the counter-intuitive associations in the prediction model, subsequent risk predictions would not be biased; these counter-intuitive associations are not expected to change when assessed in future patients selected the same way as our development cohort. However, the association of established risk factors with outcome may become weaker and as a consequence add little to the predictive performance of the model ${ }^{14}$. 
A second factor that can explain the lower performance of the model in predicting recurrence in a new cohort is the use of data imputation in the development cohort. The data collected retrospectively from patient charts were often incomplete, and we did not always succeed in extracting important data from the databases in the participating hospitals. To prevent the loss of many patient records in conjunction with list-wise deletion, we used regression imputation to replace the missing values with values estimated from associations between all relevant quantitative variables, including the outcome variable ${ }^{15}$. Although this method has proved to be superior to other imputation methods provided that values are missing completely at random (MCAR) or missing at random (MAR) conditional on other variables in the model, it is not guaranteed to give unbiased estimates of regression coefficients. If data are not missing at random (MNAR), this method is associated with a high risk of bias (Chapter 8). Unfortunately, it is impossible to empirically identify a MNAR mechanism.

Thirdly, the disappointing performance of our prediction model may be partly due to the case definition we used for our study. We limited ourselves to women with a history of early-onset PE/HELLP, aimed to predict recurrence of exactly this clinical entity. Our case definition may be just one phenotype of a much larger, quite heterogeneous, placental syndrome. The current view is that the pathophysiology of $\mathrm{PE}$ and other maternal placental syndromes are in part overlapping, in particular with respect to placental insufficiency/dysfunction ${ }^{16}$. Other phenotypes of maternal placental syndromes are pregnancy-induced hypertension, placental abruption, eclampsia, and foetal growth restriction. Limiting our case definition to recurrent PE only implied that many patients who did not develop recurrent PE but another phenotype of the placental syndrome were considered to have completed a normal pregnancy. Conversely, women with a previous maternal placental syndrome other than PE may have been wrongly excluded from this study. With these inferences, we emphasize that incomplete insight into the pathogenesis of the target disease interferes with the development of a prediction model by disabling not only the clear demarcation of the outcome variable, but also that of the inclusion criteria.

Our external validation efforts exemplify the importance of this phase in the development of a clinical prediction model. Although the necessity of a new model's external validation has been stressed by others ${ }^{17-20}$, relatively few validations have actually been performed ${ }^{20}$. Prediction studies only subjected to internal validation steps are by far more common. Internal validation can be assessed by, amongst many other techniques, split-sample validation ${ }^{21}$, cross-validation ${ }^{21}$, jackknifing ${ }^{22,23}$, and bootstrapping ${ }^{23,24}$. The latter is considered most efficient since it uses all available data and, for each bootstrap sample, uses as many records as the original dataset. Internal validation gives an estimate of the likely performance of the model in similar cohorts, but by using the same data as used for the model's development can be expected to result in too optimistic results, especially in small datasets ${ }^{25}$. Only an external validation will provide an estimate of the likely performance of the prediction model when used in practice, given that the external validation is tested on similar patients as the setting it is implemented. It goes without saying that external validity should always be proved before the introduction of a model in routine care. 
Impact of using the model for antenatal care stratification

After the model's development and internal validation, we aimed to evaluate its impact as a decision aid for the stratification of women into risk-based antenatal care management options. We determined a risk threshold value used to stratify women into risk-based strata (i.e. either below or at the threshold, or above the threshold), and designed antenatal care protocols according to the recurrence risk of early-onset PE or HELLP. The group of women below or at the threshold was allocated to receive medium-extensive antenatal care (Medium Care, or MC), as opposed to women above the threshold, who would receive more extensive care (High Care, or HC), and particularly more frequent foetal/maternal surveillance. If, during the course of pregnancy, clinical signs would indicate the imminent development of a relevant pregnancy complication, the care provider was allowed to deviate from the care protocol initially adopted. This care guided by recurrence risk (RGC) was compared to care as usual (CAU), as dictated by local protocol.

Using data from the PreCare study, we noticed that caregivers do alter their patient management according to the model's predicted risk. Even after correcting for pregnancy complications and duration, the two risk groups ( $\mathrm{MC}$ and $\mathrm{HC}$ ) differed from one another by two extra outpatient visits and various extra laboratory tests in the high-risk group. Compared to patients who were not stratified with respect to their recurrence risk (i.e. the CAU group), we did not observe more pregnancy complications and more negative foetal outcomes. In addition, self-reported psychological well-being did not differ between the CAU and RGC groups, on average.

\section{Discussion of the findings}

It follows that this evidence-based risk stratification of care functions well, and helps tailor healthcare spending to the risk profile of the individual patient. This not only reduces the burden of excessive surveillance in the medium-risk group without a higher rate of complications or self-reported psychological well-being. It is also expected to lead to lower overall healthcare expenditure for this particular medical problem.

The PreCare study was designed as a before-after study (Chapter 3). As a consequence, in the first part of the prospective enrolment of pregnant women we only recorded frequency of antenatal care and the pregnancy outcome variables (i.e. care-as-usual, or $\mathrm{CAU})$, whereas in the second part, we introduced the prediction model and management care protocols (i.e. recurrence-risk-guided care, or RGC). A randomized controlled trial would have been the preferred design for an impact analysis. For PreCare this would have implied that CAU and RGC should have been offered by random allocation either to patients or to hospitals. However, randomizing at the patient level could have been greatly influenced by contamination ${ }^{26}$. Gynaecologists would be able to apply the same knowledge about the risk of recurrence of early-onset PE or HELLP in the care as usual group as in the recurrence risk guided care group, and possibly use this knowledge as a guide for the intensity of antenatal care, diminishing any possible effects. We did not choose to randomize at the hospital level either, because of the perceived risk of large differences between local guidelines in each 
hospital. Therefore, we chose to perform a before-after study with each participating hospital being involved in both the before and the after part of the study.

\section{Conclusion and directions for future research}

We have shown that risk-based antenatal care for pregnant women with a history if early-onset PE or HELLP is feasible and does not cause more psychological or somatic morbidity compared to standard care according to local protocol. In addition, women did not report more symptoms of depression, post-traumatic stress syndrome and anxiety, and reported comparable health-related quality of life. The model we used for evaluating recurrence-risk guided care turned out to perform poorly in these women (chapter 4). In order for evidence-based risk stratification to be implemented in routine obstetrical practice, risks derived from the prediction model ought to be accurate.

A challenge for future research would be to develop a model performing adequately in differentiating women according to their recurrence risk. This model would preferably contain pre- and early pregnancy predictors so that prediction and stratification can be performed at the end of the first trimester of pregnancy. The model we developed may be used as starting point that needs to be developed further to increase performance. To improve this model's predictive performance, it might be updated with variables that independently contribute to the model's discriminative ability and calibration measures. Promising additional pre-pregnancy available predictor variables are previous occurrence of placental abruption ${ }^{27}$, maternal thrombophilia ${ }^{28}$, highdensity lipoprotein (HDL) cholesterol ${ }^{12}$, total plasma volume ${ }^{29,30}$, and homeostasis model assessment of insulin resistance (HOMA-IR) $)^{31}$. In addition, the case definition could be extended to include other phenotypes of the maternal placental syndrome, such as pregnancy-induced hypertension, placental abruption and foetal growth restriction. Obviously, because of the incompletely unravelled pathophysiology of PE together with its heterogeneous clinical presentation, it will be difficult at present to develop a robust prediction model.

\section{Part 2: Methodology and statistics regarding prediction studies}

The second part of this thesis deals with methodological and statistical challenges of prediction modelling research. Results of each chapter will be summarized, and implications and directions for future research will be discussed.

\section{Validating prediction models for avoidable risks}

In chapter 6 we demonstrate that a change in the population's composition resulting from the implementation of a prediction model will also affect the subsequent external validation, giving rise to a certain bias that resembles work-up bias in diagnostic accuracy studies. Measures of sensitivity and specificity are altered, while positive- and negative predictive values remain unbiased. This implicates that external validation of such a model should be performed in a sample not beforehand subjected to the predicted risk, at least not before the choice to be at risk (e.g. to try for next 
pregnancy) is made. However, we only provided a proof of principle based on an illustration using hypothetical numbers.

To remove the bias resulting from risk-based selective fertility from the realm of simulations, empirical examples ought to be evaluated. It should be compared to the condition without the possibility of this type of selection. A randomized controlled trial is the preferred approach to generate an unbiased estimate of the difference in estimates the performance of the model in the external validation. Any trial that studies the implementation of a prediction model or other form of risk estimation and subsequent counselling can be used for this analysis, provided that the participants are free to choose whether or not they accept to be at risk for a certain outcome. An example used in this thesis is that of accepting the exposure to a risk for a certain pregnancy complication simply by choosing to conceive. Other examples include choosing either repeat caesarean section or trial of labour after risk-based counselling for uterine rupture in multiparous women with a history of a caesarean birth ${ }^{32}$, or choosing mode of therapy after being counselled about the risk of complications or side-effects.

\section{Introducing bias by selecting patients based on previous disease}

Chapter 7 elaborates on estimating recurrence risk factors in a population of subjects with a history of first occurrence of the certain pregnancy complication. We show that estimates can be biased towards the null, even when biological or pathophysiological mechanisms are comparable. This implies that estimates from any recurrence risk study confined to subjects with a history of a first occurrence may be biased and the results ought to be interpreted with this in mind. Results can be difficult to use for etiologic purposes, yet for prognostic modelling they may be just fine. Take for example the association between BMI and the risk of recurrent PE (Chapter 2). When evaluated as a risk factor for first occurrence, BMI proves to be a strong positive risk factor $^{33}$. However, when its association with recurrent disease is studied, the association is much weaker. Naïve interpretation of these findings could result in risk counselling without bearing $\mathrm{BMI}$ in mind, since results suggest it is not a risk factor for recurrence. However, the role of BMI in the pathophysiological pathway may still be the same and as important as for first occurrence of disease. For risk prediction, the added value of predictor variables to the performance of the model is of highest concern. If it adds value, even though it shows counterintuitive estimates, it can be validly added to the model. Nevertheless, selection of these biased variables based on statistical significance or effect size will reduce the probability of being included in a model, as a direct result of being closer to the null value compared to the unbiased estimate in studies of first occurrence.

Studies of recurrence risk are larded with empirical examples of potentially biased estimates because of being restricted to patients with a prior event ${ }^{34-38}$. These often counter-intuitive findings may be explained by index-event bias, but they may also be related to relevant differences between pathophysiology of occurrence and that of recurrence. To date, there are no reports comparing the condition in which bias occurs to an unbiased situation, thereby providing empirical proof of this type of bias. 
Therefore, empirical studies should be performed to provide actual proof and more insight in the size and direction of index-event bias using real patient data instead of hypothetical simulated data. To do so, data of patients who experienced a first occurrence, and are therefore at risk for recurrences, could be combined with data of subjects at risk for first occurrence of disease.

\section{Imputing data when assumptions are unclear}

In Chapter 8 we show that multiple imputation strategies to substitute missing values may cause biased results when data are not MCAR or MAR. We also demonstrate that sometimes using only complete cases may be superior to (multiple) imputation to estimate unbiased model parameters. Our results implicate that establishing the mechanism that led to data to be missing (i.e. MCAR, MAR and MNAR) is paramount in determining what method should be used to handle missing data and to assess the possibility of biased results and the potential size and sign of bias.

Methods to determine the underlying mechanism that may cause data missing have been subject to exploration in only few (simulation) studies ${ }^{39-42}$. Results of these studies suggest that MCAR and MAR can be distinguished from one another. However, the most important challenge is to discriminate between MNAR and both other mechanisms, because MNAR is the mechanism that leads to the type of missing data that is most difficult to handle, and almost universally causing biased results. Discriminating between these two mechanisms is not trivial and depends on mostly untestable assumptions ${ }^{43}$. Contextual information may prove vital in determining what caused records to be incomplete. Future research should focus on more qualitative methods when the cause of missing data cannot be identified. 


\section{References}

1. Gynaecologie NVvOe. Hypertensieve aandoeningen in de zwangerschap2011.

2. Hernandez-Diaz S, Toh S, Cnattingius S. Risk of pre-eclampsia in first and subsequent pregnancies: prospective cohort study. Bmj. 2009;338:b2255.

3. van Rijn BB, Hoeks LB, Bots ML, Franx A, Bruinse HW. Outcomes of subsequent pregnancy after first pregnancy with early-onset preeclampsia. Am J Obstet Gynecol. Sep 2006;195(3):723-728.

4. Delahaije DH, Smits LJ, Van Kuijk SM, et al. Care as usual for pregnant women with a history of early-onset preeclampsia: an investigation of health care consumption, costs and pregnancy outcomes in tertiary referral centers in the Netherlands. Submitted.

5. Grove WM, Zald DH, Lebow BS, Snitz BE, Nelson C. Clinical versus mechanical prediction: a meta-analysis. Psychol Assess. Mar 2000;12(1):19-30.

6. Bramham K, Briley AL, Seed P, Poston L, Shennan AH, Chappell LC. Adverse maternal and perinatal outcomes in women with previous preeclampsia: a prospective study. Am J Obstet Gynecol. Jun 2011;204(6):512 e511-519.

7. Langenveld J, Buttinger A, van der Post J, Wolf H, Mol BW, Ganzevoort W. Recurrence risk and prediction of a delivery under 34 weeks of gestation after a history of a severe hypertensive disorder. BJOG. Apr 2011;118(5):589-595.

8. Langenveld J, Jansen S, van der Post J, Wolf H, Mol BW, Ganzevoort W. Recurrence risk of a delivery before 34 weeks of pregnancy due to an early onset hypertensive disorder: a systematic review. American journal of perinatology. Aug 2010;27(7):565-571.

9. McDonald SD, Best C, Lam K. The recurrence risk of severe de novo pre-eclampsia in singleton pregnancies: a population-based cohort. BJOG. Nov 2009;116(12):1578-1584.

10. Melamed N, Hadar E, Peled Y, Hod M, Wiznitzer A, Yogev Y. Risk for recurrence of preeclampsia and outcome of subsequent pregnancy in women with preeclampsia in their first pregnancy. J Matern Fetal Neonatal Med. Nov 2012;25(11):2248-2251.

11. Rasmussen S, Irgens LM, Albrechtsen S, Dalaker K. Predicting preeclampsia in the second pregnancy from low birth weight in the first pregnancy. Obstet Gynecol. Nov 2000;96(5 Pt 1):696-700.

12. Sep SJ, Smits LJ, Prins MH, Spaanderman ME, Peeters LL. Simple prepregnant prediction rule for recurrent early-onset hypertensive disease in pregnancy. Reprod Sci. Jan 2009;16(1):80-87.

13. Dahabreh IJ, Kent DM. Index event bias as an explanation for the paradoxes of recurrence risk research. JAMA. Feb 23 2011;305(8):822-823.

14. Smits L, van Kuijk SM, Leffers P, Peeters LL, Prins MH, Sep SJ. Index event bias-a numerical example. J Clin Epidemiol. Feb 2013;66(2):192-196.

15. Allison PD. Missing Data. lowa: Sage; 2001.

16. Granger JP, Alexander BT, Llinas MT, Bennett WA, Khalil RA. Pathophysiology of hypertension during preeclampsia linking placental ischemia with endothelial dysfunction. Hypertension. Sep 2001;38(3 Pt 2):718-722.

17. Altman DG, Royston P. What do we mean by validating a prognostic model? Statistics in Medicine. 2000;19(4):453-473.

18. Laupacis A, Sekar N, Stiell IG. Clinical prediction rules. A review and suggested modifications of methodological standards. JAMA. Feb 12 1997;277(6):488-494.

19. McGinn TG, Guyatt GH, Wyer PC, Naylor CD, Stiell IG, Richardson WS. Users' guides to the medical literature: XXII: how to use articles about clinical decision rules. Evidence-Based Medicine Working Group. JAMA. Jul 5 2000;284(1):79-84.

20. Moons KG, Royston P, Vergouwe $Y$, Grobbee DE, Altman DG. Prognosis and prognostic research: what, why, and how? Bmj. 2009;338:b375.

21. Steyerberg EW. Clinical Prediction Models. New York: Springer; 2009. 
22. Rothpearl A. The jackknife technique in statistical analysis. Chest. Apr 1989;95(4):940.

23. Efron B. Bootstrap Methods: Another Look at the Jackknife. The Annals of Statistics. 1979;7(1):1-26.

24. Efron B, Tibshirani R. An Introduction to the Bootstrap. New York: Chapman \& Hall; 1993.

25. Bleeker SE, Moll HA, Steyerberg EW, et al. External validation is necessary in prediction research: a clinical example. J Clin Epidemiol. Sep 2003;56(9):826-832.

26. Grobbee DE, Hoes AW. Clinical Epidemiology: Principles, Methods, and Applications for Clinical Research. Sudbury: Jones and Bartlett; 2009.

27. Melamed N - Hadar E, Hadar E - Peled Y, Peled Y - Hod M, Hod M - Wiznitzer A, Wiznitzer A - Yogev Y, Yogev Y. Risk for recurrence of preeclampsia and outcome of subsequent pregnancy in women with preeclampsia in their first pregnancy. 20121015 2012(14764954 (Electronic)).

28. Facchinetti F, Marozio L, Frusca T, et al. Maternal thrombophilia and the risk of recurrence of preeclampsia. Am J Obstet Gynecol. Jan 2009;200(1):46 e41-45.

29. Aardenburg R, Spaanderman ME, van Eijndhoven HW, de Leeuw PW, Peeters LL. A low plasma volume in formerly preeclamptic women predisposes to the recurrence of hypertensive complications in the next pregnancy. J Soc Gynecol Investig. Dec 2006;13(8):598-603.

30. Scholten RR, Sep S, Peeters L, Hopman MT, Lotgering FK, Spaanderman ME. Prepregnancy low-plasma volume and predisposition to preeclampsia and fetal growth restriction. Obstet Gynecol. May 2011;117(5):1085-1093.

31. Hauth JC, Clifton RG, Roberts JM, et al. Maternal insulin resistance and preeclampsia. Am J Obstet Gynecol. Apr 2011;204(4):327 e321-326.

32. Ophir E, Odeh M, Hirsch Y, Bornstein J. Uterine rupture during trial of labor: controversy of induction's methods. Obstet Gynecol Surv. Nov 2012;67(11):734-745.

33. Sibai B, Dekker G, Kupferminc M. Pre-eclampsia. Lancet. Feb 26-Mar 4 2005;365(9461):785-799.

34. Christiansen SC, Cannegieter SC, Koster T, Vandenbroucke JP, Rosendaal FR. Thrombophilia, clinical factors, and recurrent venous thrombotic events. Jama. May 18 2005;293(19):2352-2361.

35. Hankey GJ, Jamrozik K, Broadhurst RJ, et al. Long-term risk of first recurrent stroke in the Perth Community Stroke Study. Stroke. Dec 1998;29(12):2491-2500.

36. Gruberg L, Weissman NJ, Waksman R, et al. The impact of obesity on the short-term and long-term outcomes after percutaneous coronary intervention: the obesity paradox? J Am Coll Cardiol. Feb 20 2002;39(4):578-584.

37. Kent DM, Thaler DE. Is patent foramen ovale a modifiable risk factor for stroke recurrence? Stroke. Oct 2010;41(10 Suppl):S26-30.

38. Rich JD, Cannon CP, Murphy SA, Qin J, Giugliano RP, Braunwald E. Prior aspirin use and outcomes in acute coronary syndromes. J Am Coll Cardiol. Oct 19 2010;56(17):1376-1385.

39. Park T, Davis CS. A test of the missing data mechanism for repeated categorical data. Biometrics. Jun 1993;49(2):631-638.

40. Park T, Lee SY. A test of missing completely at random for longitudinal data with missing observations. Stat Med. Aug 30 1997;16(16):1859-1871.

41. Little RJA. A Test of Missing Completely at Random for Multivariate Data with Missing Values. Journal of the American Statistical Association. 1988;83(404):1198-1202.

42. Ridout MS, Diggle PJ. Testing for Random Dropouts in Repeated Measurement Data. Biometrics. 1991;47(4):1617-1621.

43. Curran D, Bacchi M, Schmitz SF, Molenberghs G, Sylvester RJ. Identifying the types of missingness in quality of life data from clinical trials. Stat Med. Mar 15-Apr 15 1998;17(57):739-756. 
Nederlandstalige samenvatting 
Dit proefschrift bestaat uit twee aan elkaar verwante delen. Het eerste deel behandelt de ontwikkeling van een model dat de kans op een herhaling van vroege preëclampsie schat, alsook de validatie van dit predictiemodel in een andere groep van zwangere vrouwen, en de impact die het gebruik van het model heeft op patiëntenzorg en zwangerschapsuitkomsten. In het tweede deel worden een drietal methodologische problemen die de ontwikkeling of toepassing van een predictiemodel (maar niet beperkt zijn tot deze toepassing) kunnen compliceren onder de loep genomen.

In hoofdstuk 1 van dit proefschrift worden de zwangerschapscomplicaties preëclampsie en het HELLP-syndroom beschreven en epidemiologische gegevens gepresenteerd over het vóórkomen van deze complicaties in de eerste en daaropvolgende zwangerschap. De nadruk ligt op het schatten van het recidiefrisico, nadat de eerste zwangerschap door preëclampsie en/of het HELLP-syndroom gecompliceerd raakte. Aan bod komen het gebrek aan uniformiteit in de zorg voor deze vrouwen en de mogelijkheid om op basis van de berekende recidiefkans d.m.v. een predictiemodel het zorgaanbod tijdens de vervolgzwangerschap te laten afhangen van de hoogte van die recidiefkans. Daarnaast worden een aantal potentiële valkuilen die kunnen opspelen bij de ontwikkeling van een dergelijk predictiemodel behandeld.

\section{Deel 1. Het voorspellen van een herhaling van vroege preeclampsie}

In hoofdstuk 2 wordt besproken hoe een predictiemodel werd ontwikkeld gebruikmakend van een aantal eenvoudig meetbare variabelen, met als doel de kans op een vroeg recidief preëclampsie of het HELLP-syndroom $\left(<34^{\text {ste }}\right.$ zwangerschapsweek) te berekenen. De volgende predictoren zijn meegenomen in het predictiemodel: body-mass index (BMI), nuchter bloed glucose, het al dan niet hebben van chronische hypertensie, het al dan niet gebaard hebben van een dysmatuur kind na de vorige zwangerschap (geboortegewicht $<10^{\mathrm{e}}$ percentiel), en het al dan niet doorgemaakt hebben van een intra-uteriene vruchtdood tijdens die voorgaande zwangerschap. Het model is intern gevalideerd door de regressiecoëfficiënten te vermenigvuldigen met een "penalisatie-factor". Deze factor, die altijd tussen 0 en 1 ligt, is verkregen door bootstrapping. Na interne validatie was de oppervlakte onder de receiver operating characteristic curve ('area under the curve', of AUC, een maat voor het vermogen van het model om te discrimineren tussen diegenen die wel en niet een recidief ontwikkelen) ongeveer $65 \%$. Naast dit redelijke discriminatieve vermogen bleek het model klinisch relevante subgroepen te kunnen onderscheiden gebruikmakend van een risico-afkappunt. Hierdoor konden laag- en hoog-risico zwangeren van elkaar worden gescheiden.

De opzet van de PreCare studie, waarin toepassing van het predictiemodel centraal staat, wordt beschreven in hoofdstuk 3. In dit hoofdstuk wordt uitgelegd hoe gegevens deels prospectief en deels retrospectief werden verzameld van zwangere vrouwen die in hun vorige zwangerschap preëclampsie of HELLP doormaakten, en bevielen vóór de $37^{\text {ste }}$ zwangerschapsweek. Met deze gegevens werd de externe validiteit van het 
predictiemodel vastgesteld, alsook de effecten, de kosten, en de kosteneffectiviteit van het aanbieden van zorg dat past bij het geschatte risico op een recidief (dat is óf louter gemiddeld-intensieve zorg óf hoog-intensieve zorg, naar gelang de kans op herhaling). De kosten en de kosteneffectiviteit van de toepassing van het predictiemodel worden in dit proefschrift verder niet behandeld.

Hoofdstuk 4 beschrijft de externe validatie van het predictiemodel in de patiëntenpopulatie die voor de PreCare studie werden geworven, welke bestond uit zwangeren die in hun vorige zwangerschap vóór de $34^{\text {ste }}$ zwangerschapsweek bevallen waren. Het model werd gebruikt om voor iedere vrouw de kans te schatten op een recidief. Deze vooraf geschatte kans in vergelijking met het daadwerkelijk optreden van een recidief, werd gebruikt om te bepalen of het model ook in een nieuwe steekproef van zwangere vrouwen, in staat is te discrimineren tussen ex-patiënten die wel dan niet een recidief ontwikkelen, en of de gemiddelde kans overeenkomt met de proportie recidieven in de steekproef. Het model bleek minder goed te discrimineren dan op basis van de resultaten van de interne validatie werd verwacht, en bleek groepen te maken die wat betreft werkelijk optreden van een recidief nauwelijks van elkaar verschilden. Deze resultaten geven aan dat het ontwikkelde model géén relevante toegevoegde waarde heeft bij klinische toepassing. Wellicht kan de predictieve waarde van het model verbeterd worden door een aantal kansrijke variabelen, zoals indices verkregen door 3D Doppleronderzoek van het placentabed in het eerste trimester of een aantal placenta-eiwitten, aan het model toe te voegen.

Voordat een predictiemodel in de praktijk kan worden geïntroduceerd moet het nog een belangrijke stap doorlopen, namelijk het bepalen van het effect van het gebruik van het model. In deze stap wordt geëvalueerd welke invloed het gebruik van het predictiemodel heeft op de klinische praktijk en, uiteraard, op de zwangerschapsuitkomsten. Het predictiemodel beschreven in dit proefschrift is zo aangepast dat de uitkomst van de logistische regressie-formule niet beschreven werd als de objectieve kans op een recidief vroege preeclampsie of HELLP, maar als een dichotome uitkomst. Door gebruik te maken van een afkappunt in de voorspelde kans werd de mogelijke uitkomst gedichotomiseerd tot een lage of een hoge kans op een recidief. Vervolgens kregen patiënten zorg tijdens de zwangerschap aangeboden die paste bij het vooraf berekende recidiefrisico. Diegenen met een hoge kans op een recidief kregen intensievere zorg (meer polikliniekbezoeken, meer diagnostiek om zo vroeg mogelijk het ontstaan van een recidief op te sporen) dan de groep zonder een verhoogd risico. Deze impactstudie is beschreven in hoofdstuk 5. Evaluatie van de gegevens na bevalling liet zien dat professionele zorgaanbieders binnen de verloskunde (gynaecologen en AIOS in dit specialisme), de intensiteit van zorg daadwerkelijk aanpasten aan het recidiefrisico van de patiënte; zowel in aantal polikliniekbezoeken als uitgevoerde diagnostische testen. Daarnaast kwam naar voren dat de zwangerschapsuitkomst bij vrouwen die minder intensieve zorg genoten vergelijkbaar was met die bij vrouwen in de intensieve zorg groep, ook voor wat betreft het vóórkomen van symptomen van depressie, angst en post-traumatische stress, gecorrigeerd voor het al dan niet optreden van de voorspelde herhaling van preëclampsie. 


\section{Deel 2. Methodologische en statistische problematiek bij de ontwikkeling van een predictiemodel}

In hoofdstuk 6 wordt beschreven hoe een predictiemodel dat als doel heeft de kans op een bepaalde zwangerschapsuitkomst preconceptioneel te schatten, bij externe validatie een vertekend beeld van haar werkelijke sensitiviteit en specificiteit kan geven. Dit komt voor als vrouwen opnieuw zwanger proberen te worden laten afhangen van de geschatte kans op de meestal gevreesde complicatie. Vrouwen met een hoge kans op die complicatie kunnen er voor kiezen niet meer zwanger te worden terwijl zij dit eerst wel wilden en vice versa. Het mechanisme van deze fout lijkt op de vertekening die ook in bepaald diagnostisch onderzoek voorkomt, te weten work-up bias, en in het bijzonder partial-verification bias. De vertekening die geïllustreerd wordt in dit hoofdstuk wordt besproken gebruikmakend van een aantal sprekende voorbeelden waarbij men de keuze heeft om risico te lopen. Deze situaties komen in de geneeskunde niet vaak voor. Vaak kan men enkel de mate van risico aanpassen (bijvoorbeeld een gezondere levensstijl om de kans op hart- en vaatziekten te verlagen, de kans wordt echter nooit 0 ).

Een mogelijke bron van vertekening die vooral voorkomt bij onderzoek naar recidiefkansen is index-event bias. Het mechanisme van deze "bias" wordt omschreven en geillustreerd in hoofdstuk 7. Als voor een studie naar herhalingskans personen worden geworven met een eerste voorkomen van de betreffende aandoening in de voorgeschiedenis, dan kan de samenstelling van deze groep ervoor zorgen dat belangrijke risicofactoren lijken bij te dragen aan het herhalingsrisico. Zo ontstaat een 'herhalingskans-paradox'. Als de kans op zo'n vertekening groot is, dienen de resultaten niet gebruikt te worden voor etiologie of om de patiënt adviezen te geven over, bijvoorbeeld, leefstijlaanpassingen.

Bij het uitvoeren van empirisch onderzoek is een veelvoorkomend probleem dat voor een deel van de patiënten waarden van variabelen die in de analyse worden meegenomen ontbreken. In hoofdstuk 8 wordt een stochastische simulatiestudie beschreven waarin twee uitersten van het omgaan met ontbrekende waarden in een dataset tegen elkaar af worden gezet: het analyseren van enkel alle complete rijen in een dataset, en het meermaals imputeren van alle ontbrekende waarden om een reeks complete databestanden over te houden voor analyse. De simulaties laten duidelijk zien dat niet altijd de alom geprezen meervoudige imputatie methode beter is dan de naïef beschouwde analyse van complete reeksen. Echter, er zijn geen empirische methoden beschikbaar om te bepalen dat een dergelijke situatie op een bepaalde dataset van toepassing is. Goed nadenken over mogelijke oorzaken van het ontstaan van lege cellen tijdens de dataverzameling, is dus van groot belang!

In hoofdstuk 9 worden de resultaten van de in dit proefschrift gepresenteerde studies bediscussieerd. Er wordt besproken waarom het predictiemodel ontwikkeld in deze studie, waarschijnlijk niet werkte zoals gesuggereerd werd door de derivatiestudie, maar dat een beter voorspellend model wel meerwaarde kan hebben voor de klinische praktijk. Immers, gynaecologen blijken bereid te zijn de geboden zorg aan te passen 
naargelang de berekende recidiefkans. Dit wordt gesuggereerd door het feit dat vrouwen die deelnamen aan dit onderzoek en een lage recidiefkans leken te hebben, ook minder zorg kregen dan wanneer zij niet aan dit onderzoek hadden meegedaan. Overigens had dit geen nadelige gevolgen voor de zwangerschapsuitkomst. Over het tweede deel van dit proefschrift, het gedeelte dat methodologie en statistiek betreft, wordt besproken welke belangrijke valkuilen er zijn bij (predictie-) onderzoek en hoe deze specifieke problemen kunnen worden voorkómen of opgelost. 


\section{Dankwoord}


Allereerst gaat mijn grote dank uit naar alle zwangere vrouwen die aan het PreCare onderzoek hebben meegewerkt. Zonder hun bereidheid om mee te werken had ik dit proefschrift niet kunnen schrijven.

Mijn copromotoren dr. Luc Smits en dr. Louis Peeters. Beste Luc, jij hebt aan mij de kans gegeven om me vanaf dag één te kunnen ontwikkelen als epidemioloog. Ik werd bij velerlei projecten betrokken die niets met PreCare te maken hadden waardoor ik gedwongen werd ook buiten mijn predictie-comfort-zone na te denken. Ik wil je bedanken voor het feit dat je echt een dagelijkse begeleider was, die altijd tijd maakte voor een brainstorm. Jij hebt me aangestoken met je passie voor methodologisch onderzoek, een passie die ik nu met je deel. Je gaf me de vrijheid en stimuleerde me om me als epidemioloog niet alleen te richten op het uitvoeren van onderzoek, maar ook kritisch te kijken naar onze methoden. Dit is de waardevolste les van mijn periode als promovendus. Louis, ook van jou heb ik de afgelopen jaren veel geleerd, waar ik je erg dankbaar voor ben. Jij zult als gynaecoloog wel wat meer geduld hebben moeten opbrengen dan dat je gewend was, om mij de pathologie van preeclampsie uit te leggen. Onze discussies over de rol van epidemiologisch onderzoek voor de klinische praktijk hebben mijn ogen doen openen, en ervoor gezorgd dat ik de waarde van een resultaat niet enkel van epidemiologisch en statistisch oogpunt benader. De laatste maanden van mijn onderzoek ben ik zeer geholpen door jouw uitgebreide en zeer opbouwende manier van feedback geven op mijn geschreven stukken. Mede dankzij jou zijn alle krappe deadlines van deze laatste periode gehaald. Ik kan je hier niet goed genoeg voor bedanken.

Prof. dr. Martin Prins en prof. dr. Marc Spaanderman, mijn promotoren. Martin, ik wil je bedanken voor jouw positieve en relativerende kijk op de zaken. Dit heeft er meer dan eens voor gezorgd dat ik een bespreking weer (redelijk) zorgeloos verliet. Tevens heb ik veel van je mogen leren tijdens de methodologische focus, waar je een onuitputtelijke bron van zowel medische als epidemiologische kennis bent. Marc, gedurende de loop van het PreCare onderzoek zorgde jij dat het Radboud Ziekenhuis verreweg de hoogste inclusiesnelheid behaalde. Zonder die inspanning waren we waarschijnlijk nu nog niet klaar met de werving van patiënten. Na deze periode ben je nauw betrokken geweest bij alle artikelen die de resultaten van deze studie beschrijven, en heb je me veel betrokken bij studies van promovendi van de afdeling gynaecologie. Bedankt hiervoor.

Daarnaast wil ik prof. dr. Carmen Dirksen bedanken. Beste Carmen, als promotor van Denise zag ik je bijna alleen tijdens de zeer frequente PreCare-vergaderingen. Ondanks dat ik zelf nooit kosteneffectiviteit analyses heb gedaan, heb ik veel geleerd van je inbreng hierover tijdens de meetings.

Denise, het heeft ons veel energie gekost om de studie goed op de rails te krijgen en daar te houden, en ondertussen als twee diplomaten iedereen tevreden te houden. Dat laatste lukte lang niet altijd, maar daar konden we gelukkig achteraf altijd om lachen. Ik heb onze samenwerking als heel prettig ervaren, en daarnaast waren onze werkoverleggen na een half uur nuttig vaak nog anderhalf uur erg gezellig als we 
praatten over geplande vakanties en andere zaken die wat minder met PreCare te maken hadden.

Dr. Liesbeth Scheepers, beste Liesbeth, ik ben je erg dankbaar dat je mij hebt betrokken bij de SIMPLE-studie, ondanks dat jullie al een epidemioloog in de groep hadden. Nooit heb ik zoveel gevarieerde en leuke statistische uitdagingen in één studie bij elkaar gezien, en daarnaast hield het in dat ik voor PreCare de zo belangrijke verlenging kreeg. Ellen, door de SIMPLE-studie was ik meteen verbonden aan jouw promotieonderzoek. Jij hebt een groot autodidactisch vermogen als het om methodologie en statistiek gaat, zodat ik er vaak alleen nog bij werd gevraagd om over je schouder mee te kijken. Wat jij in een korte tijd allemaal op papier hebt gezet is om trots op te zijn.

Chahinda, al vroeg tijdens mijn promotieonderzoek werden wij (meermaals) aan elkaar geïntroduceerd en sindsdien hebben we veel samengewerkt. Ik hoop dat we in de toekomst nog veel projecten samen op kunnen pakken, en dat ik, samen met Leandra, nog vaak van de Libanese keuken mag komen genieten. Ik wil je bedanken voor alles dat je mij hebt geleerd over preeclampsie en andere hypertensieve aandoeningen van de zwangerschap, en voor de fijne samenwerking.

Grote dank gaat uit naar de medewerkers van het verloskundig consortium en de gynaecologen en arts-assistenten, zonder wie de dataverzameling onmogelijk zou zijn geweest. Yvonne, bedankt dat je me al die vrijdagen van de laatste maanden steeds op sleeptouw hebt genomen en geholpen hebt met alle formaliteiten, en daarnaast bedankt voor de mooie lay-out van mijn proefschrift. Leden van de Journal Club Maastricht, de Journal Club Mesch, en van de methodologische focus wil ik bedanken voor alle leerzame discussies.

Mijn ex-kamergenotes Daniëlle, Patty en Dianne. Jarenlang hebben we gebrainstormd over hoe een dankwoord kort en krachtig opgeschreven kan worden, zodat het op één pagina past. Die hoop heb ik inmiddels opgegeven. Ik wil jullie bedanken voor alle gezelligheid, inhoudelijke en vooral de niet-inhoudelijke gesprekken en alle heerlijke etentjes waar we eigenlijk veel eerder mee hadden moeten beginnen.

Pap en mam, jullie hebben mij altijd gestimuleerd om te studeren, en daar alle mogelijkheid en vrijheid voor gegeven. Ik ben jullie daar enorm dankbaar voor. Jeroen en Bianca, bedankt voor de interesse die jullie altijd hebben getoond in mijn werk als promovendus. De laatste jaren waren jullie telkens nieuwsgierig naar waar ik allemaal mee bezig was, ik hoop dat dit boekje een antwoord daarop is.

Leandra, ik wil je bedanken voor het ontwerpen van het PreCare-logo en het mooie design van mijn cover. Op meer persoonlijke noot ben ik je dankbaar dat ik met jou in de buurt nooit aan werk heb kunnen denken. Vaak staan op deze plek ook nog schulden spijtbetuigingen over de avonden, weekenden en zelfs vakanties en huwelijksreizen die door de promovendus werden opgeofferd ten koste van hun partner. Ik heb altijd voor jou gekozen, en daar heb ik geen spijt van. 
Curriculum vitae 


\section{About the author}

Sander Martijn Job van Kuijk was born on January the $7^{\text {th }}, 1981$, in Heerlen, the Netherlands.

After graduating from secondary school (Bernardinuscollege, Heerlen) in 2000, he studied medical engineering (Biometrie) at Hogeschool Zuyd in Heerlen. He combined his last year of studying with courses in Calculus at the University of Eindhoven and Statistics and Epidemiology at Maastricht University. In 2006, he started studying Epidemiology at Maastricht University, while working in Haarlem for the Legionnaires' disease source identification unit at the Streeklaboratorium voor de Volksgezondheid Kennemerland and the Rijksinstituut voor Volksgezondheid en Milieu (RIVM).

After graduating, he started working as a teacher at the department of Epidemiology of Maastricht University, and as a PhD student at the department of Obstetrics and Gynaecology of the Maastricht University Medical Centre. He was supervised by dr. Luc Smits, dr. Louis Peeters, prof. dr. Marc Spaanderman and prof. dr. Martin Prins. This resulted in the work presented in this thesis. Sander currently works as an epidemiologist at the regional Community Health Service (GGD Zuid Limburg). 


\section{List of publications}

\section{Peer-reviewed publications}

Schoorel EN, van Kuijk SM, Melman S, Nijhuis JG, Smits LJ, Aardenburg R, de Boer K, Delemarre FM, van Dooren IM, Franssen MT, Kaplan M, Kleiverda G, Kuppens SM, Kwee A, Lim FT, Mol BW, Roumen FJ, Sikkema JM, Smid-Koopman E, Visser H, Woiski M, Hermens RP, Scheepers HC. Vaginal birth after a caesarean section: the development of a Western European population based prediction model for at term deliveries. BJOG, in press.

Schoorel EN, Melman S, van Kuijk SM, Grobman WA, Kwee A, Mol BW, Nijhuis JG, Smits JM, Aardenburg R, de Boer K, Delemarre FM, van Dooren IM, Franssen MT, Kleiverda G, Kaplan M, Kuppens SM, Lim FT, Sikkema JM, Smid-Koopman E, Visser H, Vrouenraets FP, Woiski M, Hermens RP, Scheepers HC. Predicting successful intended vaginal delivery after previous caesarean section: external validation of two prediction models in a Dutch nationwide registration-based cohort with a high intended vaginal delivery rate. $\mathrm{BJOG}$, in press.

Schoorel EN, Vankan E, Scheepers HC, Augustijn B, Dirksen CD, de Koning M, van Kuijk SM, Kwee A, Melman S, Nijhuis JG, Aardenburg R, de Boer K, Hasaart T, Mol BW, Nieuwenhuijze $M$, van Pampus MG, van Roosmalen J, Roumen F, de Vries R, Wouters M, van der Weijden T, Hermens RP. Involving women in personalised decisionmaking on mode of delivery after caesarean section: the development and pilot test of a patient decision aid. BJOG, in press.

van der Heyden JL, van Kuijk SM, van der Ham DP, Notten KJ, Janssen T, Nijhuis JG, Willekes C, Porath M, van der Post JA, Halbertsma F, Pajkrt E, Mol BW. Subsequent pregnancy after preterm prelabor rupture of membranes before 27 weeks' gestation. Am J Perinatol, in press.

van Teeffelen S, Pajkrt E, van Kuijk SM, Mol BW, Willekes C. Transabdominal amnioinfusion for improving fetal outcomes after oligohydramnios secondary to preterm prelabour rupture of membranes before 26 weeks. Cohrane. Cochrane Database Syst Rev. 2013 Aug 3.

van der Heyden JL, van der Ham DP, van Kuijk SM, Notten KJ, Janssen T, Willekes C, Nijhuis JG, Halbertsma F, , van der Post JA, Mol BW, Pajkrt E. Outcome of pregnancies with preterm prelabour rupture of membranes before 27 weeks' gestation: a retrospective cohort study. European Journal of Obstetrics \& Gynecology and Reproductive Biology. Eur J Obstet Gynecol Reprod Biol. 2013 Jul 8. 
Schoorel EN, van Kuijk SM, Nijhuis JG, Smits LJ, Scheepers HC; cesarean Section IMPLEmentation (SIMPLE) II study group. Re: Predicting successful vaginal birth after Cesarean section using a model based on Cesarean scar features examined using transvaginal sonography. Ultrasound Obstet Gynecol. 2013 Jul;42(1):122-3.

Ghossein-Doha C, Peeters LL, van Heijster S, van Kuijk SM, Spaan J, Delhaas T, Spaanderman ME. Hypertension after preeclampsia is preceded by changes in cardiac structure and function. Hypertension. 2013 Jun 3.

van Balen VA, Spaan JJ, Ghossein C, van Kuijk SM, Spaanderman ME, Peeters LL. Early Pregnancy Circulatory Adaptation and Recurrent Hypertensive Disease: An Explorative Study. Reprod Sci. 2013 Feb 18.

Smits LJ, van Kuijk SM, Leffers P, Peeters LL, Prins MH, Sep SJ. Index event bias-a numerical example. J Clin Epidemiol. 2013 Feb;66(2):192-6.

Ghossein-Doha C, van Kuijk SM, Spaanderman ME, Delhaas T, Peeters LL. Age-related alterations in cardiac geometry in formerly preeclamptic women and healthy parous controls: an explorative study. Reprod Sci. 2013 Jan;20(1):39-44.

Mostard RL, van Kuijk SM, Verschakelen JA, van Kroonenburgh MJ, Nelemans PJ, Wijnen PA, Drent M. A predictive tool for an effective use of (18)F-FDG PET in assessing activity of sarcoidosis. BMC Pulm Med. 2012 Sep 14;12:57.

Robbe EJ, van Kuijk SM, de Boed EM, Smits LJ, van der Wurff AA, Kruitwagen RF, Pijnenborg JM. Predicting the coexistence of an endometrial adenocarcinoma in the presence of atypical complex hyperplasia: immunohistochemical analysis of endometrial samples. Int J Gynecol Cancer. 2012 Sep;22(7):1264-72.

van Kuijk SM, Nijdam ME, Janssen KJ, Sep SJ, Peeters LL, Delahaije DH, Spaanderman M, Bruinse HW, Franx A, Bots ML, Langenveld J, van der Post J, van Rijn BB, Smits L. A model for preconceptional prediction of recurrent early-onset preeclampsia: derivation and internal validation. Reprod Sci. 2011 Nov;18(11):1154-9.

van Kuijk SM, Sep SJ, Nelemans PJ, Smits LJ. How long do preconception risk prediction models hold? Influence of selective fertility on model performance. Paediatr Perinat Epidemiol. 2010 Nov;24(6):602-7.

Delahaije DH, van Kuijk SM, Dirksen CD, Sep SJ, Peeters LL, Spaanderman ME, Bruinse HW, de Wit-Zuurendonk LD, van der Post JA, Duvekot JJ, van Eyck J, van Pampus MG, van der Hoeven MA, Smits LJ. Cost-effectiveness of recurrence risk guided care versus care as usual in women who suffered from early-onset preeclampsia including HELLP syndrome in their previous pregnancy (the PreCare study). BMC Pregnancy Childbirth. 2010 Oct 11;10:60. 


\section{Submitted for publication}

van Kuijk SM, Delahaije DH, Dirksen CD, Scheepers HC, Spaanderman ME, Ganzevoort W, Duvekot JJ, Oudijk MA, van Pampus MG), von Dadelszen P, Peeters LL, and Smits LJ, for the PreCare study group. Multicenter external validation and recalibration of a model for preconceptional prediction of recurrent early-onset preeclampsia.

van Kuijk SM, Delahaije DH, Dirksen CD, Scheepers HC, Spaanderman ME, Ganzevoort W, Duvekot JJ, Oudijk MA, van Pampus MG), Peeters LL, and Smits LJ, for the PreCare study group. Multicenter impact analysis of a model for predicting recurrent earlyonset preeclampsia. Results of the PreCare study.

van Kuijk SM, Peeters LL, Viechtbauer W, Smits LJ. Bias in regression coefficient estimates when assumptions for handling missing data are violated.

Delahaije DH, Smits LJ, van Kuijk SM, Peeters LL, Scheepers HC, Spaanderman ME, Oudijk MA, Duvekot JJ, van Pampus MG, Ganzevoort WJ, Dirksen CD. Care as usual for pregnant women with a history of early-onset preeclampsia: an investigation of health care consumption, costs and pregnancy outcomes in tertiary referral centers in the Netherlands.

Schoorel EN, Hünen DR, van Kuijk SM, Augustijn BC, Dirksen CD, Kwee A, Mol BW, Nijhuis JG, Smits LJ, Hermens RP, Scheepers HC. Probability of vaginal birth after caesarean: do not ask the gynaecologist.

Schoorel EN, Melman S, van Kuijk SM, Alink M, Dirksen CD, Kwee A, Mol BW, Nijhuis JG, Smits LJ, Scheepers HC, Hermens RP. The Netherlands, high vaginal birth after caesarean rate but large practice variation.

Renes L, van Kuijk SM, Smits LJ, Donckers J, Land JS, Evers J. The long-term outcome of 946 consecutive couples visiting a fertility clinic in 2001-2003.

van der Ham DP, van Kuijk SM, Opmeer BC, Willekes C, van Beek JJ, Mulder AL, van Loon A, Groenewout M, Mantel G, Bloemenkamp KW, Porath M, Kwee A, Akerboom B, Papatsonis DN, Metz G, Nijhuis JG, Mol BW. Can neonatal sepsis be predicted in near term prelabour rupture of membranes? Development of a prediction model. 